\title{
RESOLVED NEAR-INFRARED STELLAR POPULATIONS IN NEARBY GALAXIES
}

\author{
Julianne J. Dalcanton ${ }^{1}$, Benjamin F. Williams ${ }^{1}$, Jason L. Melbourne ${ }^{2}$, Léo Girardi $^{3}$, Andy Dolphin ${ }^{4}$, \\ Philip A. Rosenfield ${ }^{1}$, Martha L. Boyer ${ }^{5}$, Roelof S. De Jong ${ }^{6}$, Karoline Gilbert $^{1}$, PaOla Marigo ${ }^{7}$, \\ Knut Olsen ${ }^{8}$, Anil C. Seth ${ }^{9,11}$, and Evan SKillman ${ }^{10}$ \\ ${ }^{1}$ Department of Astronomy, University of Washington, Box 351580, Seattle, WA 98195, USA; jd@ astro.washington.edu, ben@astro.washington.edu, \\ philrose@astro.washington.edu, kgilbert@astro.washington.edu \\ ${ }^{2}$ Caltech Optical Observatories, Division of Physics, Mathematics and Astronomy, Mail Stop 301-17, \\ California Institute of Technology, Pasadena, CA 91125, USA; jmel@ caltech.edu \\ ${ }^{3}$ Obsservatorio Astronomico di Padova-INAF, Vicolo dell'Osservatorio 3, I-35122 Padova, Italy; leo.girardi@oapd.inaf.it \\ ${ }^{4}$ Raytheon, 1151 East Hermans Road, Tucson, AZ 85706, USA; adolphin@ raytheon.com \\ ${ }^{5}$ Space Telescope Science Institute, Baltimore, MD 21218, USA; mboyer@ stsci.edu \\ ${ }^{6}$ Astrophysikalisches Institut Potsdam (AIP), An der Sternwarte 16, 14482 Potsdam, Germany; rdejong@aip.de \\ ${ }^{7}$ Dipartimento di Astronomia, Universitá di Padova, Vicolo dell'Osservatorio 2, I-35122 Padova, Italy; paola.marigo@unipd.it \\ ${ }^{8}$ National Optical Astronomy Observatory, 950 North Cherry Avenue, Tucson, AZ 85719, USA; kolsen@ noao.edu \\ ${ }^{9}$ Department of Physics and Astronomy, University of Utah, Salt Lake City, UT 84112, USA; aseth@ astro.utah.edu \\ ${ }^{10}$ Department of Astronomy, University of Minnesota, 116 Church Street SE, Minneapolis, MN 55455, USA; skillman@astro.umn.edu \\ Received 2011 May 13; accepted 2011 September 28; published 2011 December 22
}

\begin{abstract}
We present near-infrared (NIR) color-magnitude diagrams (CMDs) for the resolved stellar populations within 26 fields of 23 nearby galaxies $(\lesssim 4 \mathrm{Mpc}$ ), based on images in the $F 110 \mathrm{~W}$ and $F 160 \mathrm{~W}$ filters taken with the WideField Camera 3 (WFC3) on the Hubble Space Telescope (HST). The CMDs are measured in regions spanning a wide range of star formation histories, including both old dormant and young star-forming populations. We match key NIR CMD features with their counterparts in more familiar optical CMDs, and identify the red core helium-burning (RHeB) sequence as a significant contributor to the NIR flux in stellar populations younger than a few $100 \mathrm{Myr}$ old. The strength of this feature suggests that the NIR mass-to-light ratio can vary significantly on short timescales in star-forming systems. The NIR luminosity of star-forming galaxies is therefore not necessarily proportional to the stellar mass. We note that these individual RHeB stars may also be misidentified as old stellar clusters in images of nearby galaxies. For older stellar populations, we discuss the CMD location of asymptotic giant branch (AGB) stars in the HST filter set and explore the separation of AGB subpopulations using a combination of optical and NIR colors. We empirically calibrate the magnitude of the NIR tip of the red giant branch in $F 160 \mathrm{~W}$ as a function of color, allowing future observations in this widely adopted filter set to be used for distance measurements. We also analyze the properties of the NIR red giant branch (RGB) as a function of metallicity, showing a clear trend between NIR RGB color and metallicity. However, based on the current study, it appears unlikely that the slope of the NIR RGB can be used as an effective metallicity indicator in extragalactic systems with comparable data. Finally, we highlight issues with scattered light in the WFC3, which becomes significant for exposures taken close to a bright Earth limb.
\end{abstract}

Key words: galaxies: abundances - galaxies: distances and redshifts - galaxies: dwarf - galaxies: irregular galaxies: stellar content - infrared: stars - stars: AGB and post-AGB - stars: carbon

Online-only material: color figures

\section{INTRODUCTION}

Near-infrared (NIR) observations have become increasingly important for studies of galaxies and their evolution over cosmic time (e.g., Conselice et al. 2005; Dahlen et al. 2005; Saracco et al. 2006; Cirasuolo et al. 2010). This trend will no doubt continue during the coming decade, thanks to continued improvements in NIR detectors, the growing maturity of adaptive optics, the installation of Wide-Field Camera 3 (WFC3) on Hubble Space Telescope (HST), and the upcoming launch of the James Webb Space Telescope (JWST).

The importance of NIR observations for galaxy evolution studies rests on the reduced sensitivity of the NIR mass-to-light ratio to dust and to the age or metallicity of the underlying stellar population, particularly compared to the optical or ultraviolet. The NIR luminosity of a galaxy is therefore thought to be a robust indicator of stellar mass (e.g., Thronson \& Greenhouse 1988; Brinchmann \& Ellis 2000; Bundy et al. 2005).

\footnotetext{
${ }^{11}$ Center for Astrophysics Fellow.
}

However, the ability to correctly interpret NIR observations relies on accurate stellar population modeling, which in turn requires accurate isochrones, stellar lifetimes, and spectra for the evolving stars which dominate the flux at NIR wavelengths (see Conroy \& Gunn 2010 for an estimate of current uncertainties).

Recently, there has been some concern that the mass-to-light ratio in the NIR is not nearly as stable as has been assumed. On the theoretical side, Maraston et al. (2006) have suggested that the NIR flux from asymptotic giant branch (AGB) stars may lead to drastically lower mass-to-light ratios when intermediateage populations are present, as must be the case for in situ observations of young galaxies at high redshift (although see Kriek et al. 2010). Lower NIR mass-to-light ratios could also help to explain why some elliptical galaxy progenitors at high redshift appear to have stellar densities higher than seen in the local universe (van Dokkum et al. 2008).

The most accurate constraints on evolving stars' contribution to the NIR come from resolving the stellar populations directly (e.g., Rejkuba et al. 2006; Gullieuszik et al. 2007, 2008; Melbourne et al. 2010a, most recently). With 
WFC3's IR channel on HST, we are now able to resolve large numbers of individual stars in nearby galaxies. There are well over a hundred galaxies within the Local Volume ( $D \lesssim 4 \mathrm{Mpc}$ ) that are close enough to be targeted for resolved stellar population studies. These galaxies have already been observed extensively with HST in the optical (Dalcanton et al. 2009; K. M. Gilbert et al. 2012, in preparation), and their color-magnitude diagrams (CMDs) have revealed the galaxies to have a wide range of metallicities and star formation histories (SFHs). These systems therefore form an ideal set for probing the properties of the same stellar populations in the NIR.

To this end, we undertook an HST "snapshot" survey of nearby galaxies with existing high-quality optical data. We took advantage of the high throughput and resolving power of the WFC3 IR channel to produce CMDs of individual fields in 23 galaxies, spanning a variety of stellar populations. In this paper, we present our sample, and characterize the resulting CMDs. These data will be used in subsequent papers to place quantitative constraints on the contribution of AGB and red core helium-burning ( $\mathrm{RHeB}$ ) stars to the total luminosity (Melbourne et al. 2012), on the lifetimes of AGB stars, and on the population of carbon stars.

The outline of the paper is as follows. In Section 2 we present our sample selection, observations, reductions, and matched optical photometry. We also discuss a previously uncharacterized transient scattered light feature in the WFC3 infrared channel (hereafter WFC3/IR). In Section 3 we discuss the origin of different features in the NIR CMDs and optical-NIR color-color diagrams, and highlight the importance of RHeB stars to the overall luminosity, for populations younger than $\sim 0.5 \mathrm{Gyr}$. We discuss the properties of the tip of the red giant branch (TRGB) in the NIR (Section 4), and the AGB and red giant branch (RGB) luminosity function in Section 5. Finally, we discuss the metallicity dependence of the NIR RGB morphology and color on metallicity in Section 6.

\section{DATA}

\subsection{Sample Selection}

To characterize NIR CMDs as a function of stellar age and metallicity, we require targets whose stellar populations have been constrained using optical observations in tandem with wellcalibrated stellar isochrones. We therefore chose SNAP targets from among those nearby galaxies with archival high-quality multi-color imaging (typically from Advanced Camera for Surveys (ACS), but in some cases from WFPC2). Of these, we selected a subset of 61 fields in 51 galaxies from the ACS Nearby Galaxy Survey Treasury (ANGST), a volume-limited sample of non-Local Group galaxies out to $\sim 4 \mathrm{Mpc}$ (Dalcanton et al. 2009), and from the archival legacy program ANGRRR: Archival Nearby Galaxies: Reduce, Reuse, Recycle (K. M. Gilbert et al. 2012, in preparation). Based on their HST stellar photometry, we have verified that these target fields have (1) sufficient numbers of stars to contain hundreds of candidate AGB stars within a single WFC3/IR field of view (FOV); (2) uncrowded stellar photometry of sufficient quality to provide useful constraints on the SFH and metallicity distribution; ${ }^{12}$ (3) indications from

\footnotetext{
12 Galaxies beyond $4 \mathrm{Mpc}$ are sufficiently crowded that the deep optical photometry needed for deriving SFHs is not possible. Galaxies closer than $1 \mathrm{Mpc}$ are sufficiently extended that few AGB stars actually fall in a typical FOV and contamination from Galactic foreground sources is severe, making this study more effective in more distant galaxies.
}

the range of RGB colors that the stellar populations host a nonnegligible fraction of stars whose metallicities fall outside of the range of well-studied Large Magellanic Cloud (LMC)/Small Magellanic Cloud (SMC) metallicities; and (4) a sufficiently broad range of SFHs to allow sampling of evolving stars of different masses. Our target list included multiple pointings in the more massive systems, whenever the optical data suggested that there are significant variations in stellar age and metallicity at different locations within the galaxy.

During Cycle 17 we obtained observations for 26 of the 61 possible SNAP targets. Two galaxies (Holmberg II and NGC 2403) had multiple pointings (two and three pointings, respectively), isolating regions with different SFHs and metallicities. The properties of the 23 observed galaxies are listed in Table 1. Galaxy names, positions, apparent blue magnitudes $\left(B_{\mathrm{T}}\right)$, diameters, morphological $T$-types, and Hi line widths $\left(W_{50}\right)$ have been adopted from the primary name in the Karachentsev et al. (2004) Catalog of Neighboring Galaxies. Distance moduli are based on the $F 814 W$ TRGB from Dalcanton et al. (2009) for most galaxies, and from Karachentsev et al. (2003) for NGC 7793. Group memberships are from Karachentsev (2005) or Tully et al. (2006). Foreground extinctions $\left(A_{\mathrm{V}}\right)$ are from Schlegel et al. (1998), as reported by the online Galactic Dust Extinction Service at the Infrared Science Archive. ${ }^{13}$

\subsection{Observations}

Observations for this program were carried out during Cycle 17 as SNAP-11719. Some fraction of these were taken during the period of instrument commissioning after the Hubble repair mission. All targets were observed in both $F 110 \mathrm{~W}$ and $F 160 \mathrm{~W}$ with HST's WFC3 IR channel. These filters offer the greatest depth in a given exposure time, and thus are likely to become the workhorse filters for the WFC3/IR camera. Observations were carried out in a three-point "WFC3-IR-DITHER-LINE" pattern, with exposures in both $F 110 W$ and $F 160 W$ taken at each pointing. We adopted the STEP50 exposure sequence, which accommodates data with a large dynamic range by using both short and long non-destructive reads. We used NSAMP $=9$ in $F 110 \mathrm{~W}$ and NSAMP $=11$ in $F 160 \mathrm{~W}$, giving total exposure times of $597^{\mathrm{s}} .7$ and $897^{\mathrm{S}} .7$, respectively. The exposure sequence was interleaved to allow buffers to dump during the exposures, with no latency. To maximize the schedulability of our observations, we did not specify a roll-angle orientation constraint.

Properties of the actual observations can be found in Table 2, where we include both the name of the galaxy from Table 1 and the name of the specific target within the galaxy. Target names were chosen to match the names of existing optical data sets at the same position; these associated data sets are also listed in Table 2. The centers of the WFC3 FOVs were chosen to maximize the overlap with the optical data. The locations of the WFC3/IR footprints are shown in red in Figure 1, superimposed on Digitized Sky Survey images of each galaxy. Blue regions show the locations of the associated optical imaging from ACS or WFPC2. Because we did not specify an orientation, occasionally a small corner of the WFC3/IR FOV fell off the area covered by the optical data. This mismatch occurred in cases where the optical FOV was not centered on the most desirable part of the galaxy, and the scheduled roll angle happened to be unfavorable; however, the fraction of the

\footnotetext{
$\overline{13}$ http://irsa.ipac.caltech.edu/applications/DUST/
} 
Table 1

Sample Galaxies

\begin{tabular}{|c|c|c|c|c|c|c|c|c|c|c|}
\hline Galaxy & $\begin{array}{c}\text { Alt. } \\
\text { Names }\end{array}$ & $\begin{array}{l}\text { R.A. } \\
\text { (J2000) }\end{array}$ & $\begin{array}{l}\text { Decl. } \\
\text { (J2000) }\end{array}$ & $\begin{array}{c}\text { Diam. } \\
(/)\end{array}$ & $B_{\mathrm{T}}$ & $A_{\mathrm{V}}$ & $m-M$ & $T$ & $\begin{array}{c}W_{50} \\
\left(\mathrm{~km} \mathrm{~s}^{-1}\right)\end{array}$ & Group \\
\hline DDO53 & U4459 & 08:34:06.5 & $66: 10: 45$ & 1.6 & 14.55 & 0.118 & 27.79 & 10 & 25 & M81 \\
\hline DDO78 & & $10: 26: 27.9$ & $67: 39: 24$ & 2.0 & 15.8 & 0.066 & 28.18 & -3 & & M81 \\
\hline DDO82 & U5692 & $10: 30: 35.0$ & $70: 37: 10$ & 3.4 & 13.57 & 0.133 & 27.90 & 9 & & M81 \\
\hline HoI & U5139,DDO63 & 09:40:28.2 & 71:11:11 & 3.6 & 13.64 & 0.153 & 27.95 & 10 & 29 & M81 \\
\hline HoII & U4305 & 08:19:05.9 & $70: 42: 51$ & 7.9 & 11.09 & 0.098 & 27.65 & 10 & 66 & M81 \\
\hline HS117 & & $10: 21: 25.2$ & $71: 06: 58$ & 1.5 & 16.5 & 0.359 & 27.91 & 10 & 13 & M81 \\
\hline I 2574 & U5666,DDO81 & $10: 28: 22.4$ & $68: 24: 58$ & 13.2 & 10.84 & 0.112 & 27.90 & 9 & 115 & M81 \\
\hline KDG2 & E540-030,KK9 & $00: 49: 21.1$ & $-18: 04: 28$ & 1.2 & 16.37 & 0.072 & 27.61 & -1 & & Scl \\
\hline KDG63 & U5428,DDO71 & 10:05:07.3 & $66: 33: 18$ & 1.7 & 16.01 & 0.303 & 27.74 & -3 & 19 & M81 \\
\hline KDG73 & & $10: 52: 55.3$ & $69: 32: 45$ & 0.6 & 17.09 & 0.056 & 28.03 & 10 & 18 & M81 \\
\hline KKH37 & & $06: 47: 45.8$ & $80: 07: 26$ & 1.2 & 16.4 & 0.231 & 27.56 & 10 & 20 & \\
\hline M81 & N3031,U5318 & $09: 55: 33.5$ & 69:04:00 & 26.9 & 7.69 & 0.249 & 27.77 & 3 & 422 & M81 \\
\hline N300 & & $00: 54: 53.5$ & $-37: 40: 57$ & 21.9 & 8.95 & 0.039 & 26.5 & 7 & 149 & $14+13$ \\
\hline N404 & U718 & 01:09:26.9 & $35: 43: 03$ & 2.5 & 11.21 & 0.181 & 27.42 & -1 & 78 & \\
\hline $\mathrm{N} 2403$ & U3918 & $07: 36: 54.4$ & $65: 35: 58$ & 21.9 & 8.82 & 0.124 & 27.5 & 6 & 231 & M81 \\
\hline N2976 & U5221 & $09: 47: 15.6$ & $67: 54: 49$ & 5.9 & 11.01 & 0.224 & 27.76 & 5 & 97 & M81 \\
\hline N3077 & U5398 & $10: 03: 21.0$ & $68: 44: 02$ & 5.4 & 10.46 & 0.208 & 27.92 & 10 & 65 & M81 \\
\hline N3741 & U6572 & $11: 36: 06.4$ & $45: 17: 07$ & 2.0 & 14.38 & 0.077 & 27.55 & 10 & 81 & $14+07$ \\
\hline N4163 & U7199 & $12: 12: 08.9$ & $36: 10: 10$ & 1.9 & 13.63 & 0.062 & 27.29 & 10 & 18 & $14+07$ \\
\hline N7793 & & $23: 57: 49.4$ & $-32: 35: 24$ & 9.3 & 9.70 & 0.060 & 27.96 & 7 & 174 & Scl \\
\hline $\mathrm{Sc} 22$ & Sc-dE1 & $00: 23: 51.7$ & $-24: 42: 18$ & 0.9 & 17.73 & 0.046 & 28.11 & 10 & & Scl \\
\hline U8508 & IZw60 & $13: 30: 44.4$ & $54: 54: 36$ & 1.7 & 14.12 & 0.047 & 27.06 & 10 & 49 & $14+07$ \\
\hline UA292 & CVnI-dwA & $12: 38: 40.0$ & $32: 46: 00$ & 1.0 & 16.10 & 0.048 & 27.79 & 10 & 27 & \\
\hline
\end{tabular}

Notes. Distances, $B_{\mathrm{T}}, W_{50}$, and $T$-type taken from CNG, with updates from (Dalcanton et al. 2009) and (Karachentsev et al. 2003) for NGC 7793; group membership from Karachentsev (2005) or Tully et al. (2006); $A_{\mathrm{V}}$ from IRSA.

Table 2

Observations and Photometry

\begin{tabular}{|c|c|c|c|c|c|c|c|c|c|}
\hline \multirow{2}{*}{$\begin{array}{l}\text { Catalog } \\
\text { Name }\end{array}$} & \multirow{2}{*}{$\begin{array}{l}\text { Target } \\
\text { Name }\end{array}$} & \multirow[t]{2}{*}{ Obs. Date } & \multirow[t]{2}{*}{$N_{\text {stars }}$} & \multirow{2}{*}{\multicolumn{2}{|c|}{$\begin{array}{l}\Sigma_{\max } \quad \Sigma_{\min } \\
(\# / \text { sq. } \operatorname{arcsec})\end{array}$}} & \multicolumn{2}{|c|}{$50 \%$ Completeness } & \multirow{2}{*}{$\begin{array}{l}\text { Optical } \\
\text { PropID }\end{array}$} & \multirow{2}{*}{$\begin{array}{l}\text { Optical } \\
\text { Filters }\end{array}$} \\
\hline & & & & & & $(F 110 W)$ & $(F 160 W)$ & & \\
\hline DDO53 & UGC4459 & 2010-04-23 11:15:02 & 9366 & 2.24 & 0.07 & 26.02 & 25.02 & GO-10605 & $F 555 W, F 814 W$ \\
\hline DDO78 & DDO78 & 2010-04-20 14:41:53 & 11536 & 2.07 & 0.28 & 25.97 & 25.04 & GO-10915 & $F 475 W, F 814 W$ \\
\hline DDO82 & DDO82 & 2010-05-07 06:56:06 & 25590 & 5.51 & 0.35 & 25.71 & 24.69 & GO-10915 & $F 475 W, F 606 W, F 814 W$ \\
\hline HoI & UGC5139 & 2009-08-21 22:55:14 & 13425 & 2.13 & 0.60 & 25.98 & 25.00 & GO-10605 & $F 555 W, F 814 W$ \\
\hline HoII & UGC4305-1 & 2010-02-26 09:38:51 & 19328 & 3.53 & 0.39 & 25.72 & 24.76 & GO-10605 & $F 555 W, F 814 W$ \\
\hline HoII & UGC4305-2 & 2010-01-04 07:01:20 & 21869 & 4.85 & 0.16 & 25.79 & 24.79 & GO-10605 & $F 555 W, F 814 W$ \\
\hline HS117 & HS117 & 2010-02-24 02:05:52 & 3467 & 3.24 & 0.01 & 26.00 & 25.12 & GO-9771 & $F 606 W, F 814 W$ \\
\hline $\mathrm{I} 2574$ & IC2574-SGS & 2010-02-25 03:03:05 & 27521 & 3.81 & 0.86 & 25.62 & 24.68 & GO-9755 & $F 435 W, F 555 W, F 814 W$ \\
\hline KDG2 & ESO540-030 & 2009-12-17 12:00:32 & 3890 & 2.43 & 0.02 & 26.12 & 25.01 & GO-10503 & $F 606 W, F 814 W$ \\
\hline KDG63 & DDO71 & 2010-04-21 16:01:31 & 7316 & 2.78 & 0.02 & 25.98 & 25.03 & GO-9884 & $F 606 W, F 814 W$ \\
\hline KDG73 & KDG73 & 2010-06-09 17:46:07 & 2140 & 0.98 & 0.01 & 26.13 & 25.16 & GO-10915 & $F 475 W, F 814 W$ \\
\hline \multirow[t]{2}{*}{ ККH37 } & ККH37 & 2009-09-29 10:41:04 & 5097 & 3.77 & 0.01 & 26.05 & 25.02 & GO-10915 & $F 475 W, F 814 W$ \\
\hline & & & $\ldots$ & $\ldots$ & $\ldots$ & $\ldots$ & $\ldots$ & GO-9771 & $F 606 W, F 814 W$ \\
\hline M81 & M81-DEEP & 2010-06-13 00:21:05 & 6159 & 1.43 & 0.13 & 26.10 & 25.12 & GO-10915 & $F 475 W, F 606 W, F 814 W$ \\
\hline N300 & NGC 0300-WIDE1 & 2010-04-19 17:45:59 & 27898 & 3.66 & 1.29 & 25.36 & 24.47 & GO-10915 & $F 475 W, F 606 W, F 814 W$ \\
\hline N404 & NGC 404 & 2009-12-31 00:24:37 & 23159 & 4.00 & 0.34 & 25.71 & 24.73 & GO-10915 & $F 606 W, F 814 W(\mathrm{WFPC} 2)$ \\
\hline $\mathrm{N} 2403$ & NGC 2403-DEEP & 2010-02-28 19:16:27 & 9492 & 2.77 & 0.27 & 25.88 & 25.01 & GO-10915 & $F 606 W, F 814 W(\mathrm{WFPC} 2)$ \\
\hline N2403 & NGC 2403-HALO-6 & $2010-04-25$ 04:26:22 & 5691 & 2.03 & 0.15 & 26.07 & 25.11 & GO-10523 & $F 606 W, F 814 W$ \\
\hline $\mathrm{N} 2403$ & SN-NGC 2403-PR & 2010-04-22 07:56:14 & 38792 & 5.77 & 1.73 & 23.40 & 22.35 & GO-10182 & $F 475 W, F 606 W, F 814 W$ \\
\hline N2976 & NGC 2976-DEEP & 2010-02-25 02:03:27 & 15392 & 4.05 & 0.25 & 25.84 & 24.92 & GO-10915 & $F 475 W, F 606 W, F 814 W$ \\
\hline N3077 & NGC 3077-PHOENIX & 2010-02-21 22:49:04 & 8813 & 3.14 & 0.20 & 26.00 & 25.14 & GO-9381 & $F 435 W, F 555 W, F 814 W$ \\
\hline N3741 & NGC 3741 & 2009-11-07 01:33:13 & 6819 & 4.44 & 0.00 & 25.98 & 24.86 & GO-10915 & $F 475 W, F 814 W$ \\
\hline N4163 & NGC 4163 & $2010-03-23$ 17:39:56 & 19105 & 4.77 & 0.07 & 25.90 & 24.71 & GO-10915 & $F 475 W, F 606 W, F 814 W$ \\
\hline N7793 & NGC 7793-HALO-6 & 2010-06-14 19:11:39 & 6578 & 2.14 & 0.02 & 26.11 & 25.07 & GO-10523 & $F 606 W, F 814 W$ \\
\hline $\mathrm{Sc} 22$ & SCL-DE1 & 2009-09-08 00:45:13 & 2440 & 3.17 & 0.02 & 26.19 & 25.09 & GO-10503 & $F 606 W, F 814 W$ \\
\hline U8508 & UGC8508 & 2009-10-14 19:41:41 & 12664 & 4.38 & 0.03 & 25.97 & 24.83 & GO-10915 & $F 475 W, F 814 W$ \\
\hline UA292 & UGCA292 & 2010-05-18 12:38:27 & 2373 & 2.53 & 0.01 & 26.19 & 25.14 & GO-10915 & $F 475 W, F 814 W$ \\
\hline
\end{tabular}

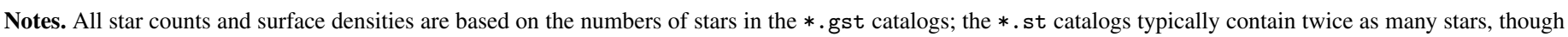

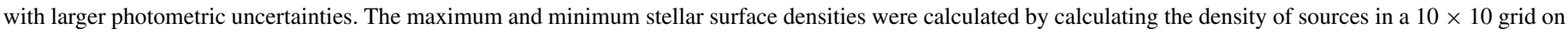
the image. 


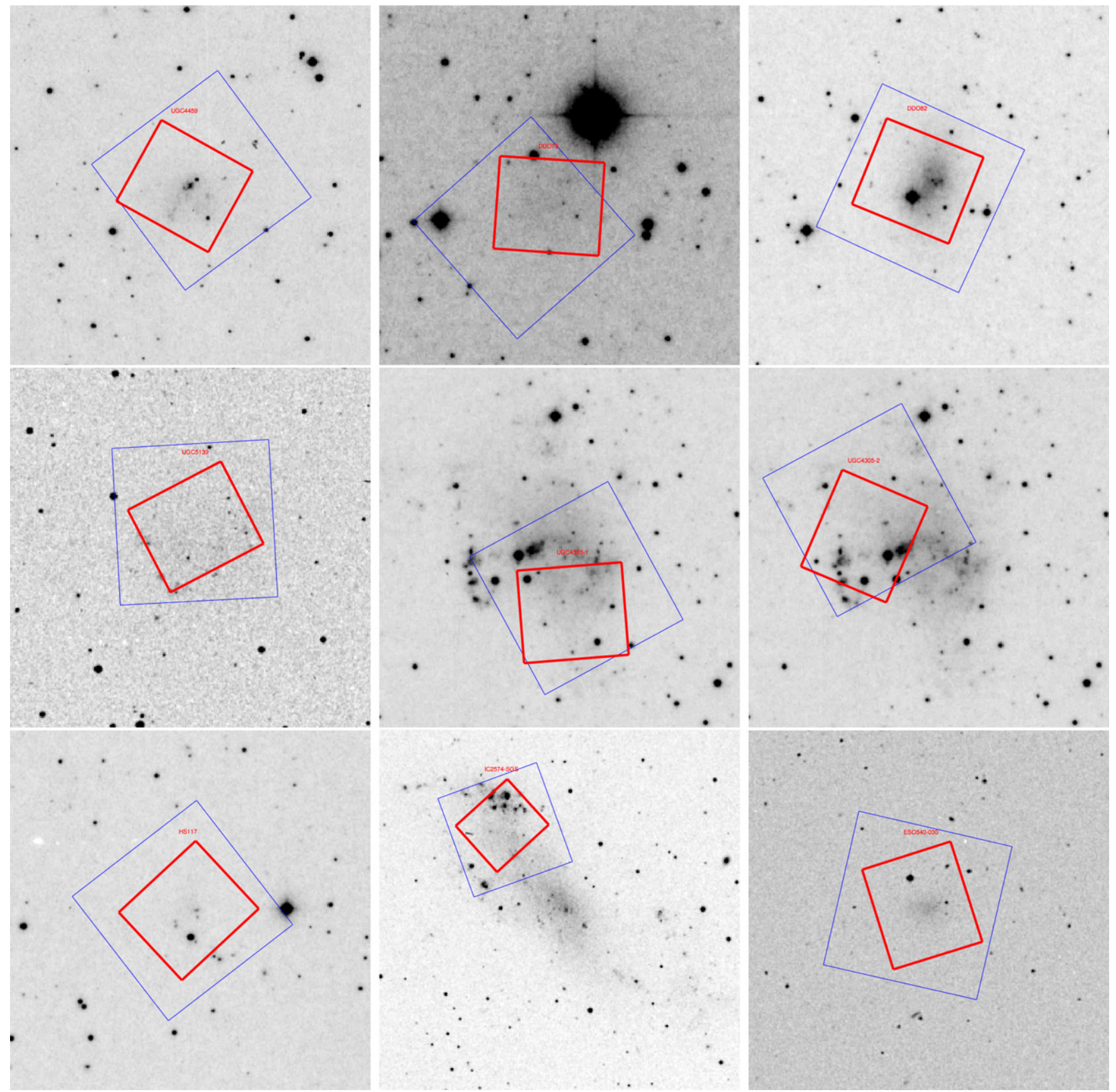

Figure 1. Position of the WFC3/IR field of view (red), overlaid on an optical image from the Digitized Sky Survey. Blue region shows the area covered by optical $H S T$ data. (Target names from upper left to lower right: (a) UGC4459; (b) DDO78; (c) DDO82; (d) UGC5139; (e) UGC4305-1; (f) UGC4305-2; (g) HS117; (h) IC2574SGS; (i) ESO540-030; (j) DDO71; (k) KDG73; (1) KKH37; (m) M81-DEEP; (n) NGC 0300-WIDE1; (o) NGC 404; (p) NGC 2403-DEEP; (q) NGC 2403-HALO-6; (r) SN-NGC 2403-PR; (s) NGC 2976-DEEP; (t) NGC 3077-PHOENIX; (u) NGC 3741; (v) NGC 4163; (w) NGC 7793-HALO-6; (x) SCL-DE1; (y) UGC8508; (z) UGCA292.)

(A color version of this figure is available in the online journal.)

WFC3/IR FOV lacking optical coverage is less than $10 \%$ in almost all cases.

In Figure 2 we show false-color images of the observations. There are a number of features to note. First, in many cases it is clear that our short exposures have already reached the "crowding limit," where stars are sufficiently close on the sky that fainter stars could not be detected, even with longer exposures; this effect can also be seen as spatially varying depth in the CMDs. Second, background galaxies are far more prevalent than in optical HST images, suggesting that unresolved background galaxies are likely to be a more significant contaminant of the NIR CMDs. Third, the images are marked with a number of circles, located at positions of "IR blobs," that are flagged as bad data by the WFC3/IR pipeline (see the WFC3-2010-06 ISR by N. Pirzkal). Fourth, extended line emission is visible in $F 110 \mathrm{~W}$ images of young star-forming regions, as can be seen in the close-up of a star-forming region in IC2574-SGS, shown in Figure 3. The line emission in this 


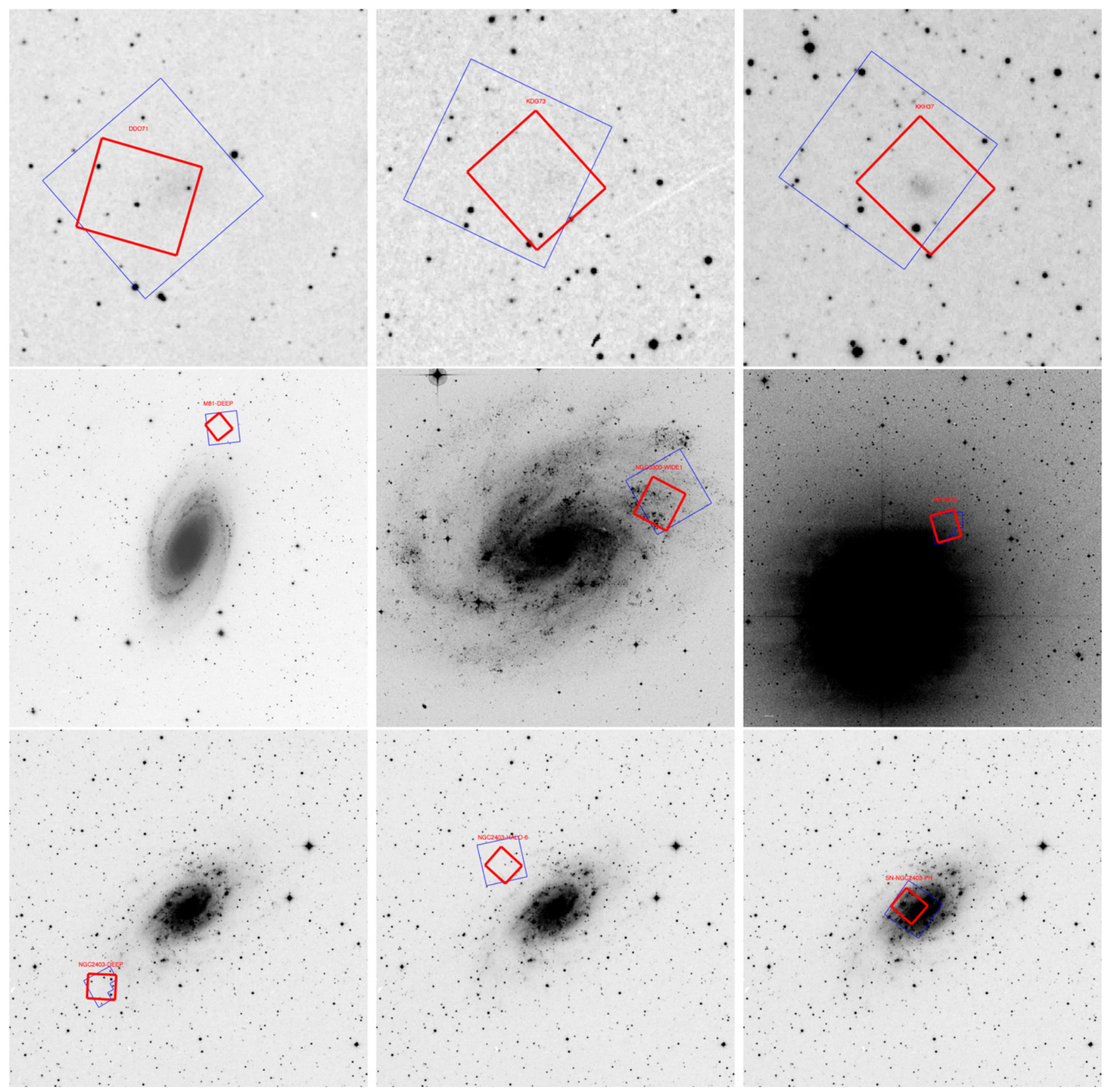

Figure 1. (Continued)

filter is dominated by the [SIII] $9069 \AA$ A, $9532 \AA$ doublet, with additional contributions from He I at $10830 \AA$ and $10833 \AA$ (from two photon emission) and Paschen- $\beta$ at $12818 \AA$.

Finally, and perhaps most concerning, a number of the images show significant scattered light, particularly in the lower left region of the image (see UGC4305-1 or NGC 2403-HALO-6, for example). We now discuss the origin of this scattered light component.

\subsubsection{Scattered Light}

Figure 4 shows the series of six combined $*$ FLT images taken during our one orbit visit, after all detected sources have been fit and subtracted from the images. The series shows images in both $F 110 \mathrm{~W}$ (exposures 1,3 , and 5 in the sequence) and $F 160 \mathrm{~W}$ (exposures 2, 4, and 6). The images show two types of structures in the "sky" background. The first is persistent structure due to unresolved stars in the image; this component does not change throughout the visit, but varies from galaxy to galaxy. The second structure (falling typically on the left-hand side of the image and peaking in the bottom left corner) varies rapidly in amplitude during a single orbit but has a consistent shape when present. The pattern of the time variation is not consistent, however, such that the brightness of the scattered light feature peaks at different times during an orbit. This variability leads to an apparent variation of the color of the scattered light; if the scattered light peaks during an $F 110 \mathrm{~W}$ exposure, the scattered light can appear blue, but if it peaks during an $F 160 \mathrm{~W}$ exposure, the light can appear red. 


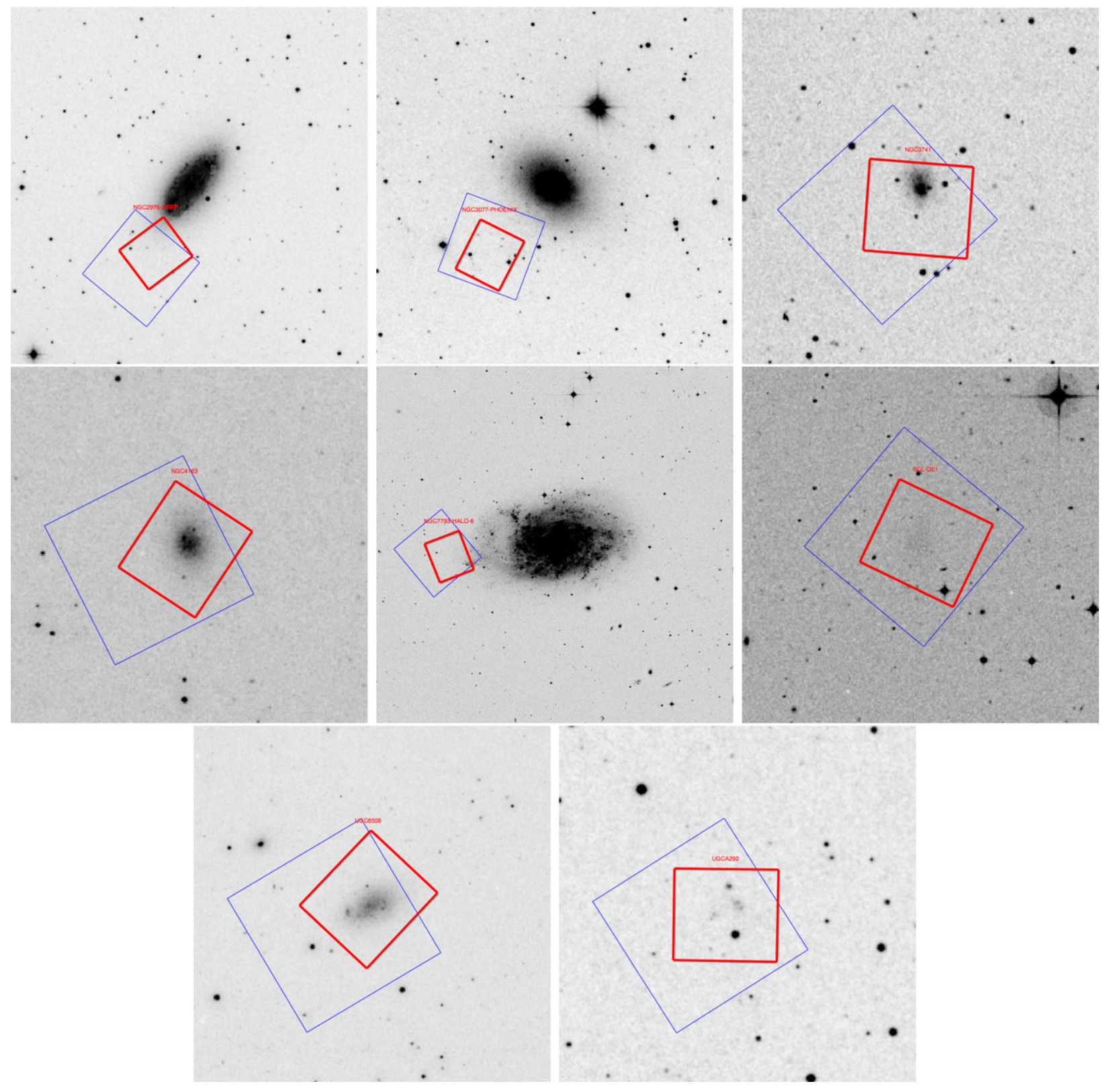

Figure 1. (Continued)

The rapid variation in the amplitude of the scattered light places strong constraints on its origin, as there are few telescope pointing attributes that vary significantly during an orbit. The angles between the principal V1 axis and the Sun or the Moon (FITS header keywords SUNANGLE and MOONANGL) vary little during an orbit, nor does the amount of zodiacal light. The only quantities which do vary on that timescale are the angle between the Sun and Earth's limb (keyword SUN_ALT) and, more directly, the angle between the spacecraft pointing and Earth's limb (keyword LOS_LIMB in the *. JIT or *. JIF files). We thank Ben Weiner (2010, private communication) for pointing out this possibility, based on his analysis of similar effects seen in WFC3/IR grism data. ${ }^{14}$

To examine the variation of the scattered light as a function of the angle to Earth's limb, we analyzed the sky levels in

${ }^{14}$ See also ISR-ACS 2003-05 by J. Biretta et al. for an analysis of ACS background light as a function of limb angle. each star-subtracted *.FLT image. We assume that there are three contributors to the star-subtracted "sky" image: (1) a mean uniform background; (2) a spatially structured, timeinvariant background due to unresolved stars; and (3) a spatially structured, time-variable background due to scattered light, which peaks in the lower left quadrant. To assess these different components, we recorded the mean level in the upper right quadrant of each image, which is assumed to be free of the scattered light feature, and in the lower left region dominated by the feature ([0:308, 0:507], in image coordinates). Note that we do not expect these two sky levels to be identical, even in the absence of the scattered light feature, because of the contribution of light from unresolved stars. However, they both should share a common "floor," representative of the mean sky background during the observation.

In the left-hand panel of Figure 5, we show the mean sky level in the upper right quadrant of each exposure, plotted as a function of the limb angle between the spacecraft and the Earth, 

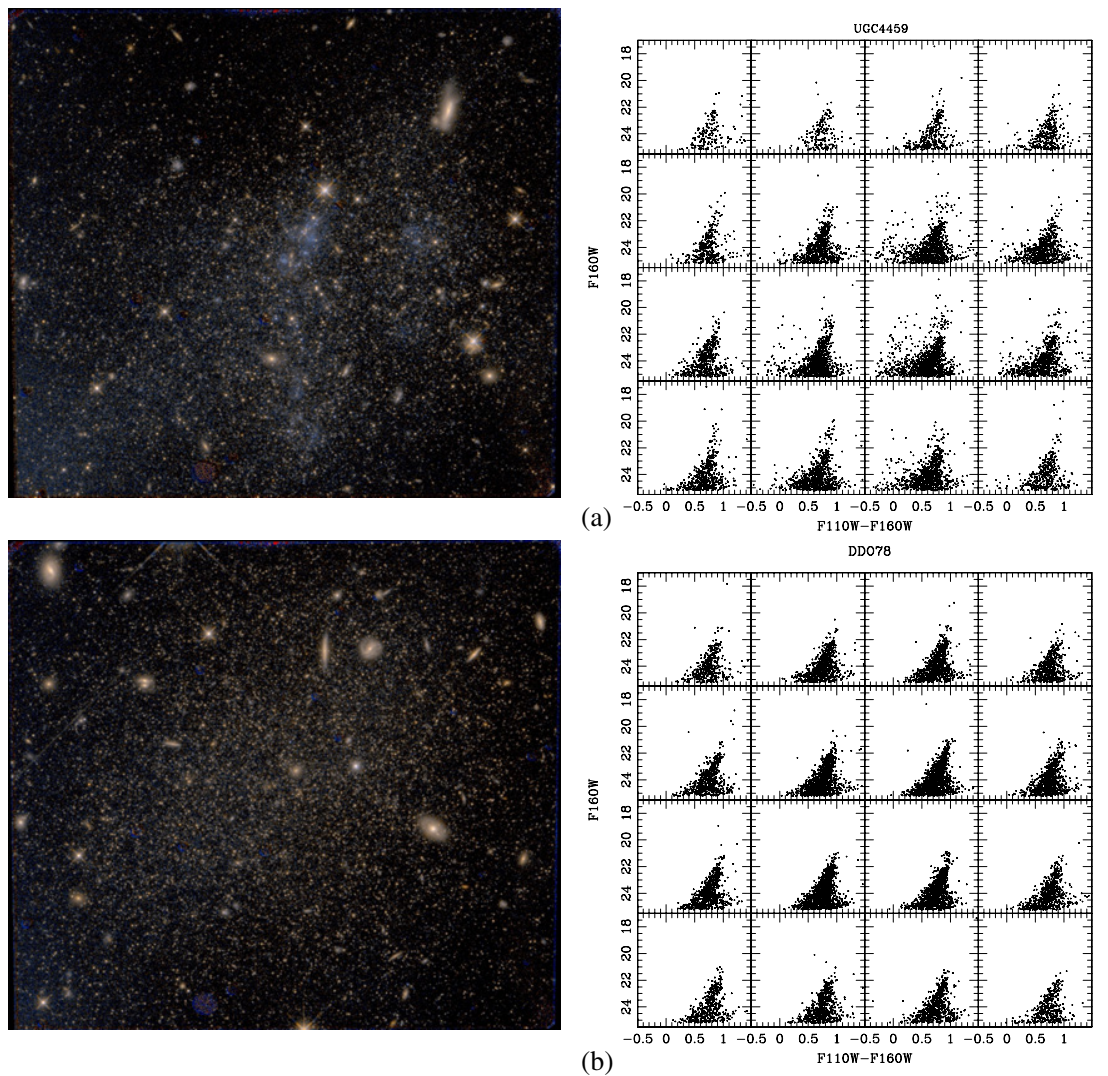

Figure 2. Left panels: false-color $F 110 W+F 160 W$ image of the WFC3/IR field. Right panels: color-magnitude diagrams generated for a grid of subregions, such that the upper left CMD corresponds to the upper left of the adjacent image. Data are shown for (a) the target UGC4459 within galaxy DDO53; (b) DDO78; (c) DDO82; (d) the target UGC5139 within galaxy HoI; (e) the target UGC4305-1 within galaxy HoII; (f) the target UGC4305-2 within galaxy HoII; (g) HS117; (h) the target IC2574-SGS within galaxy I2574; (i) the target ESO540-030 within galaxy KDG2; (j) the target DDO71 within galaxy KDG63; (k) KDG73; (l) KKH37; (m) the target M81-DEEP within galaxy M81; (n) the target NGC0300-WIDE1 within galaxy N300; (o) the target NGC404 within galaxy N404; (p) the target NGC2403-DEEP within galaxy N2403; (q) the target NGC2403-HALO-6 within galaxy N2403; (r) the target SN-NGC2403-PR within galaxy N2403; (s) the target NGC2976-DEEP within galaxy N2976; (t) the target NGC3077-PHOENIX within galaxy N3077; (u) the target NGC3741 within galaxy N3741; (v) the target NGC4163 within galaxy N4163; (w) the target NGC7793-HALO-6 within galaxy N7793; (x) the target SCL-DE1 within galaxy Sc22; (y) the target UGC8508 within galaxy U8508; (z) the target UGCA292 within galaxy UA292.

(A color version of this figure is available in the online journal.)

for the $F 110 W$ and $F 160 W$ filters, respectively. If the Earth's limb was dark at the time of observation, the sky level is plotted with an open circle. Observations taken within a single orbit are connected with a line.

From the left-hand side of Figure 5, it is clear that the sky brightness depends strongly on the limb angle for the $F 110 \mathrm{~W}$ filter, at least when the Earth's limb is bright. Below angles of $40^{\circ}$, the mean sky level increases by roughly a factor of two or more. We expect that this correlation would be significantly tighter if this test were repeated for empty fields, where there would be little contribution from unresolved sources to the measurement of the sky.

In the right-hand panel of Figure 5, we attempt to capture the amplitude of the scattered light feature visible in the first two panels of Figure 4. Unfortunately, we cannot simply track the variation of the sky level in that region, since we expect some limb-angle-dependent variation in the overall sky level, as seen in the left-hand plot of Figure 5. Instead, we look at the limb angle dependence of the excess light in the lower left, compared to the upper right (Gradient $\equiv \mathrm{Sky}_{\text {lower left }}-\mathrm{Sky}_{\text {upper right }}$ ). We assume that when the scattered light feature is absent, the difference in the sky level between the upper right and lower left regions reflects the structure in the sky due to unresolved sources. This difference should be constant with time, no matter what the mean sky background level is. For each set of observations (three per filter in a single orbit), we then look at the "excess" in the difference between the lower left and the upper right, compared to the minimum during the orbit. We then scale the resulting excess by the minimum sky level in the four quadrants of the image, to give an indication of the fractional strength of the feature, compared to the typical sky level when unresolved sources are not present.

The right-hand side of Figure 5 shows that for most of the exposures, the relative sky brightness of the lower left and upper right varies by less than $1 \%$ between exposures, as long at the angle to the bright Earth limb is greater than $30^{\circ}$. If the angle is less than $30^{\circ}$, however, a strong scattered light feature is produced in the majority of cases. The onset is quite sharp, and is present in both filters, unlike the increase in the uniform sky brightness with limb angle, which is gradual and much stronger in $F 110 \mathrm{~W}$. Based on this analysis, the galaxies with the most prominent scattered light features ( $>4 \%$ in either filter) are UGC4305-1, IGC2574-SGS, DDO71, SN-NGC 2403-PR, NGC 2403-DEEP, NGC 2403-HALO-6, and KDG73, in order 

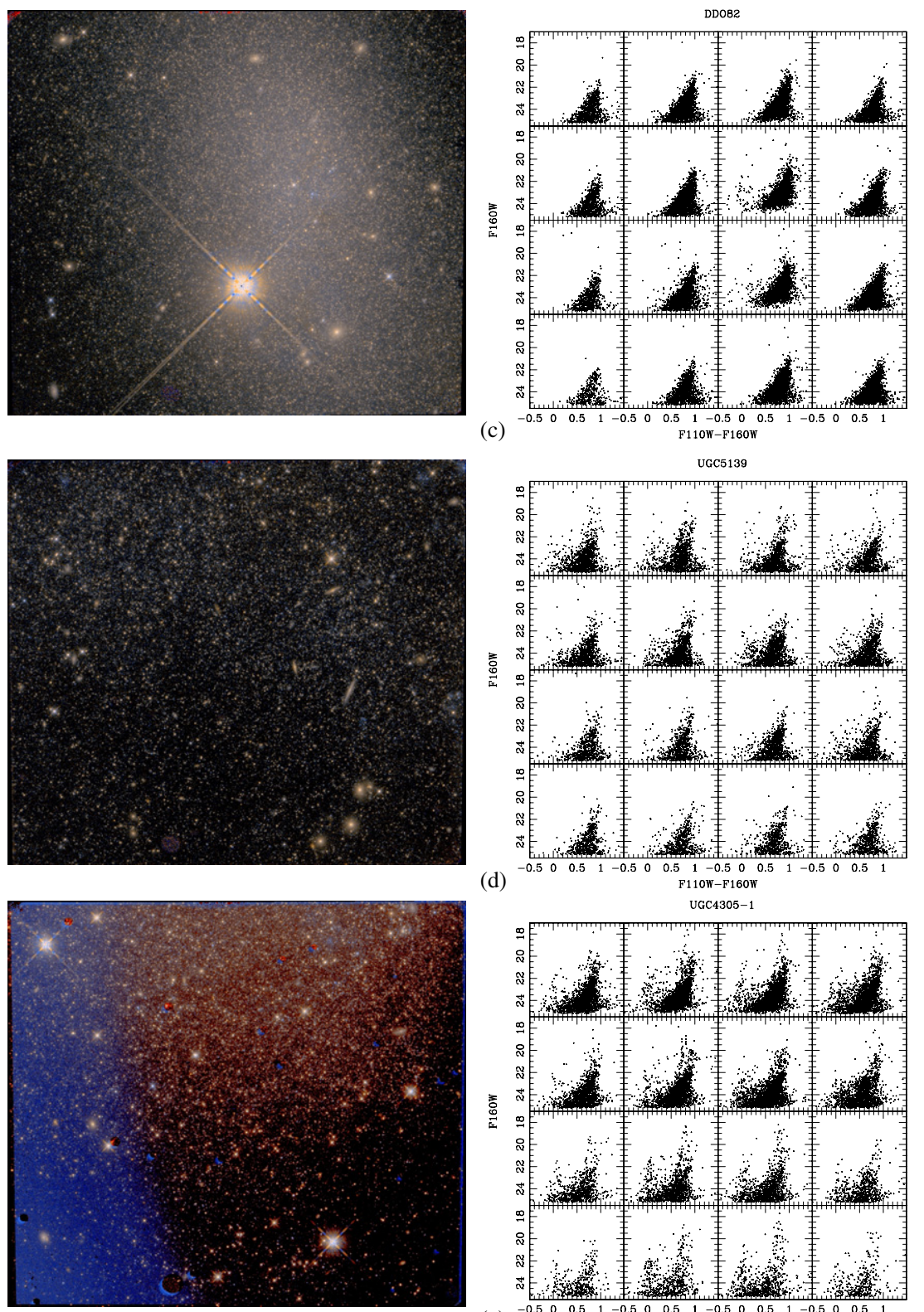

(e)
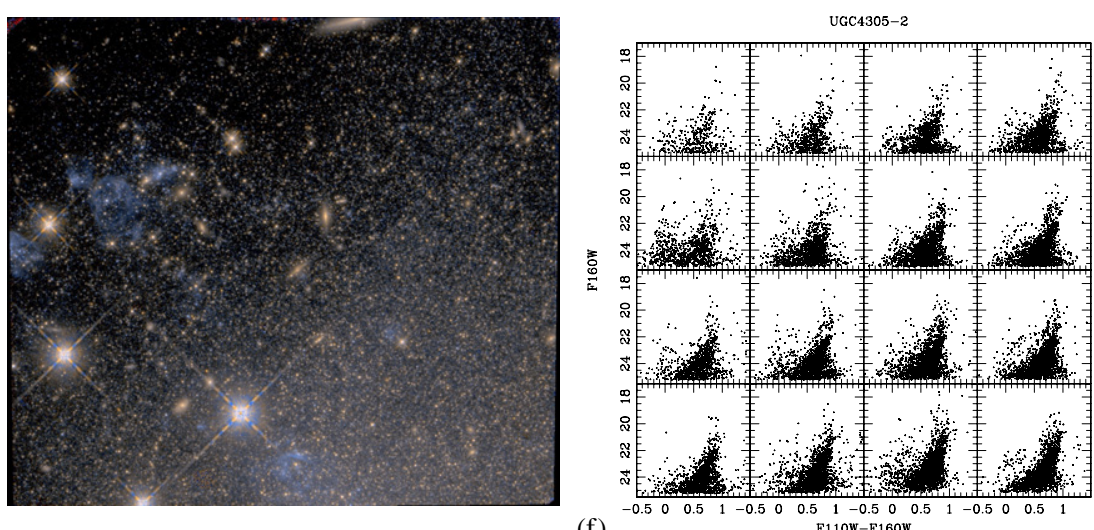

Figure 2. (Continued) 

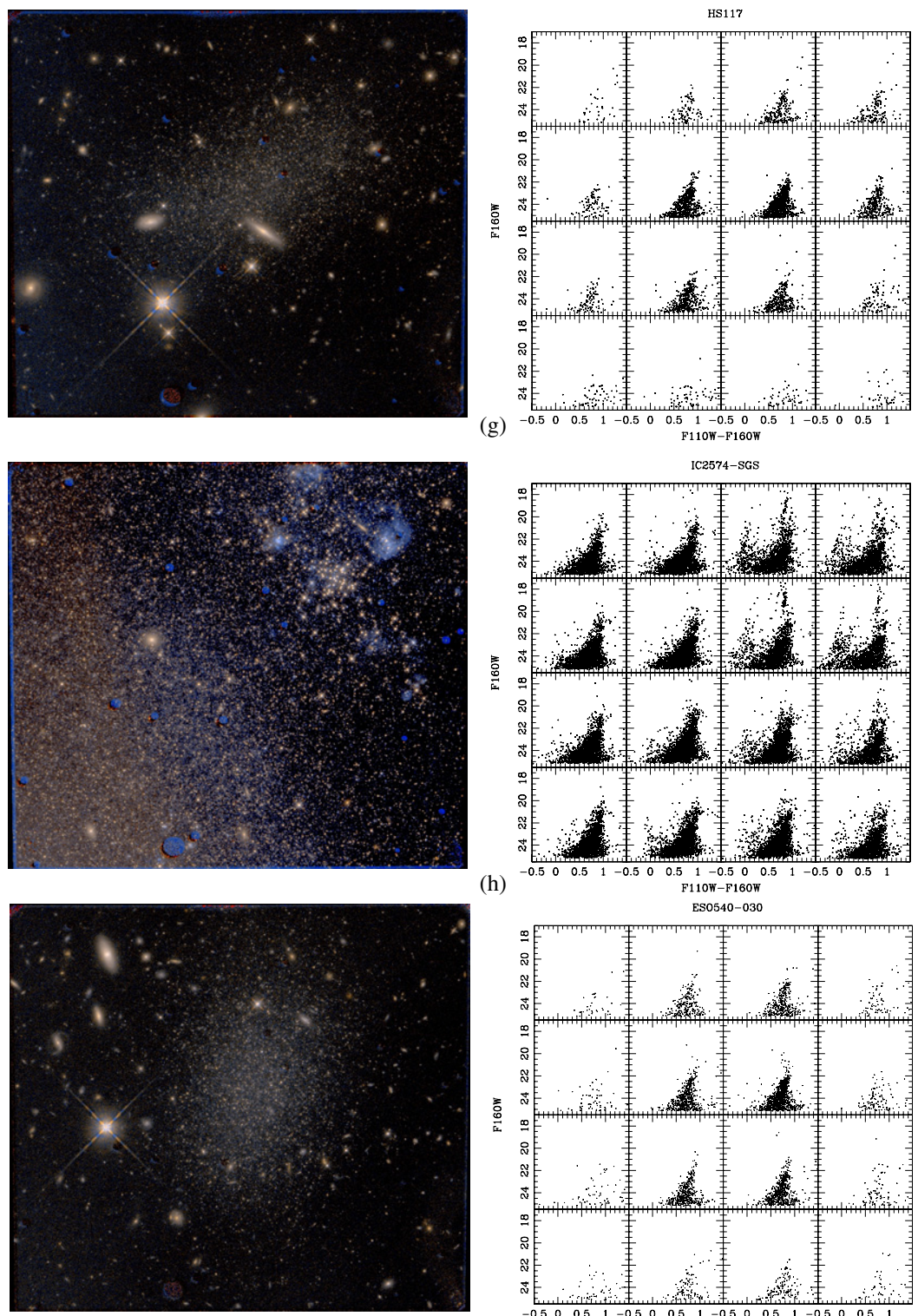

(h)
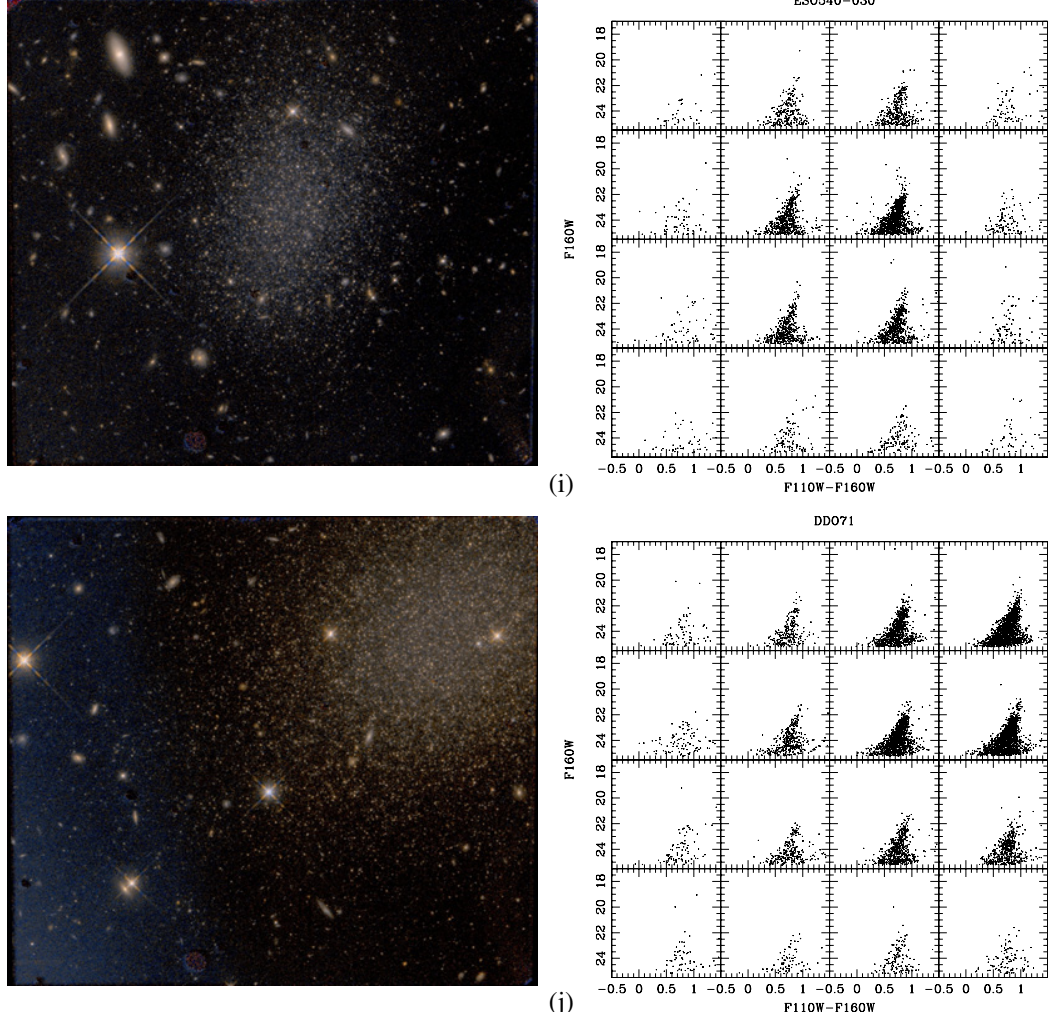

Figure 2. (Continued)

of strongest to weakest. This ranking agrees with the visual impression seen in the color images in Figure 2.

For our subsequent analysis, we make no additional attempt to compensate for the structure of the scattered light feature in these images. Since our photometry uses a very localized estimate of the sky, our results should be insensitive to this feature, except through having to accept more noise in the affected region. Given the brightness of the stellar sources, including observations with low limb angle observations produces more benefits through increased integration time than are lost through 

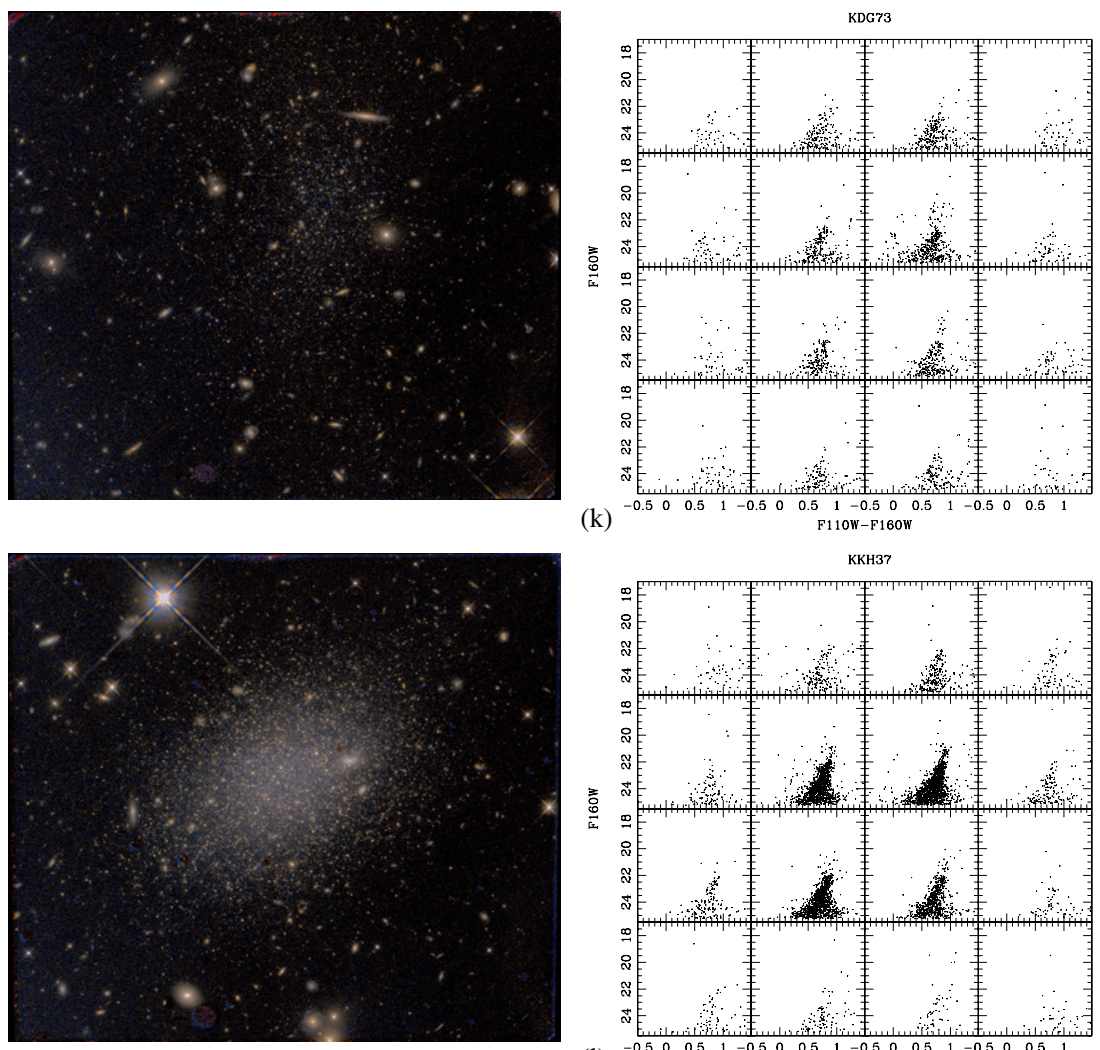

KKH37

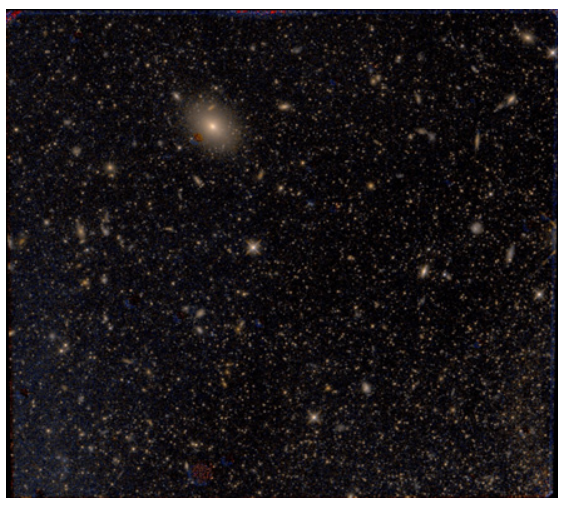

(1)

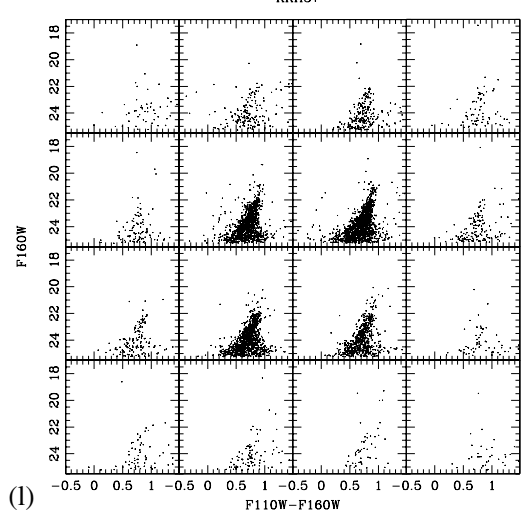

MB1-DEEP

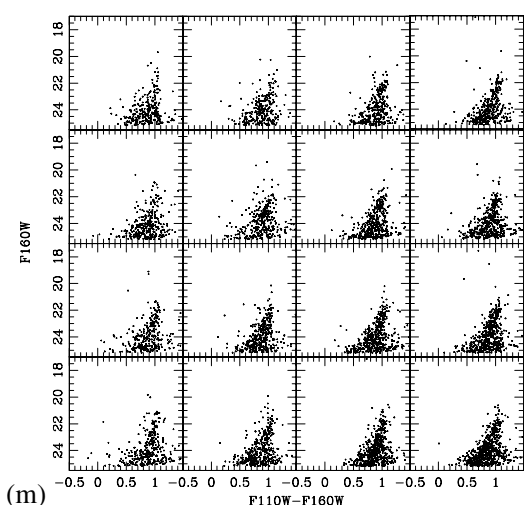

(m)
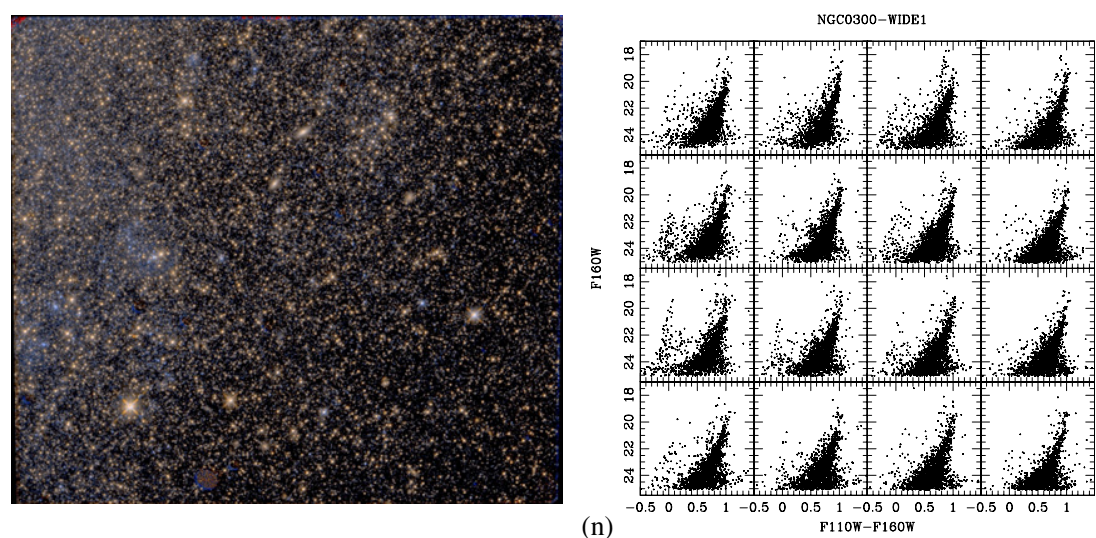

(n)

Figure 2. (Continued) 

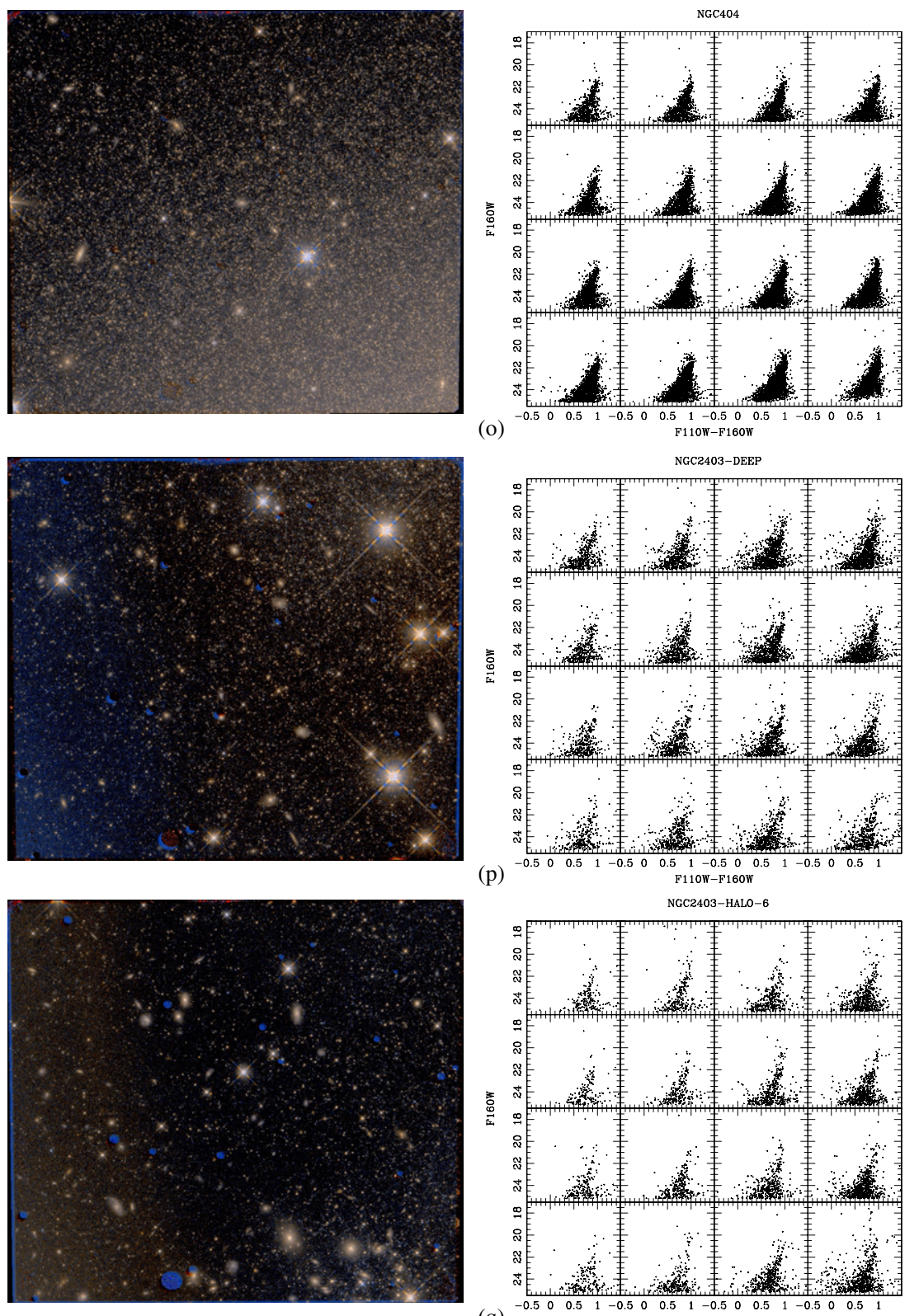

(q)
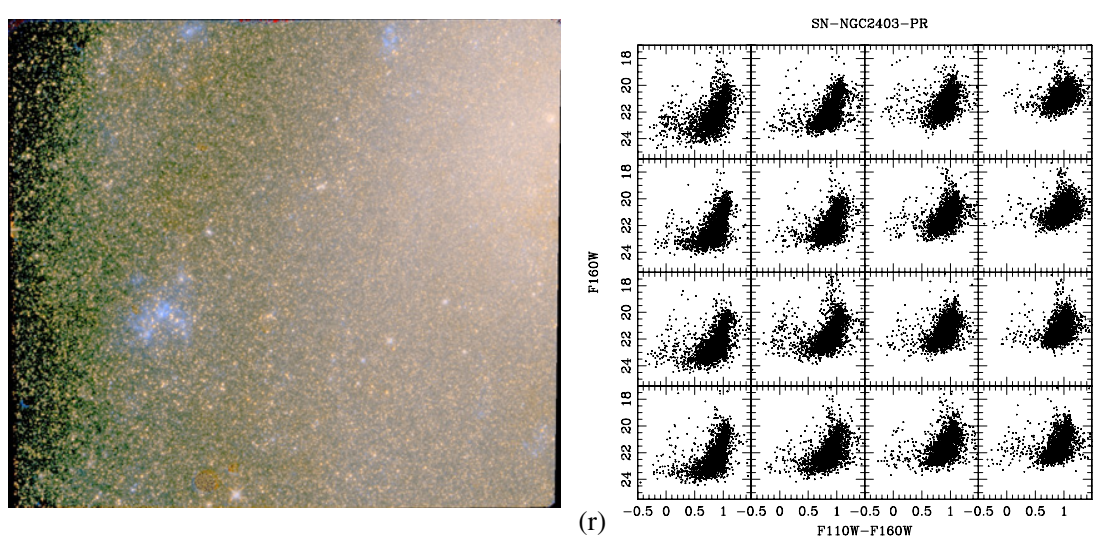

Figure 2. (Continued) 

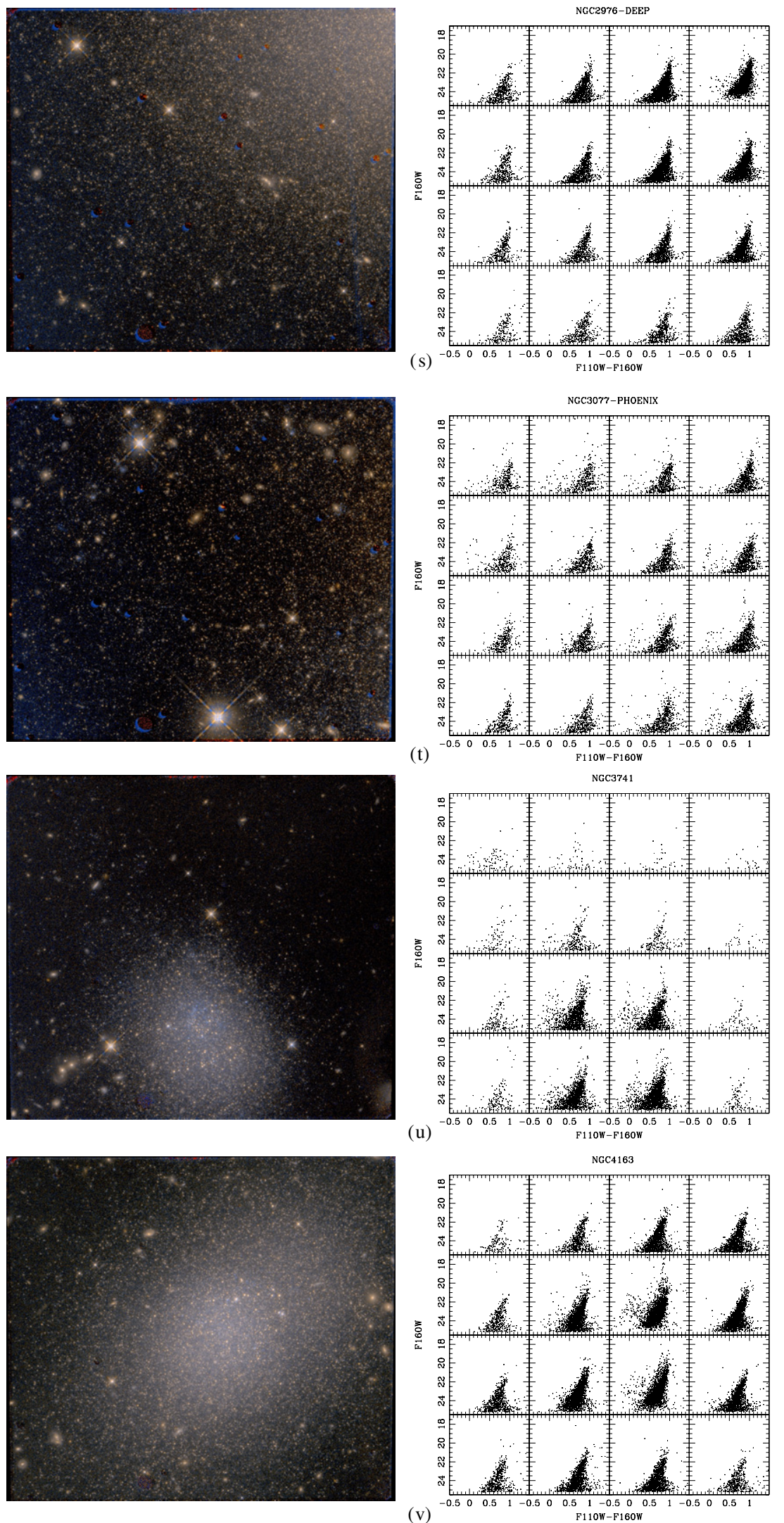

Figure 2. (Continued) 

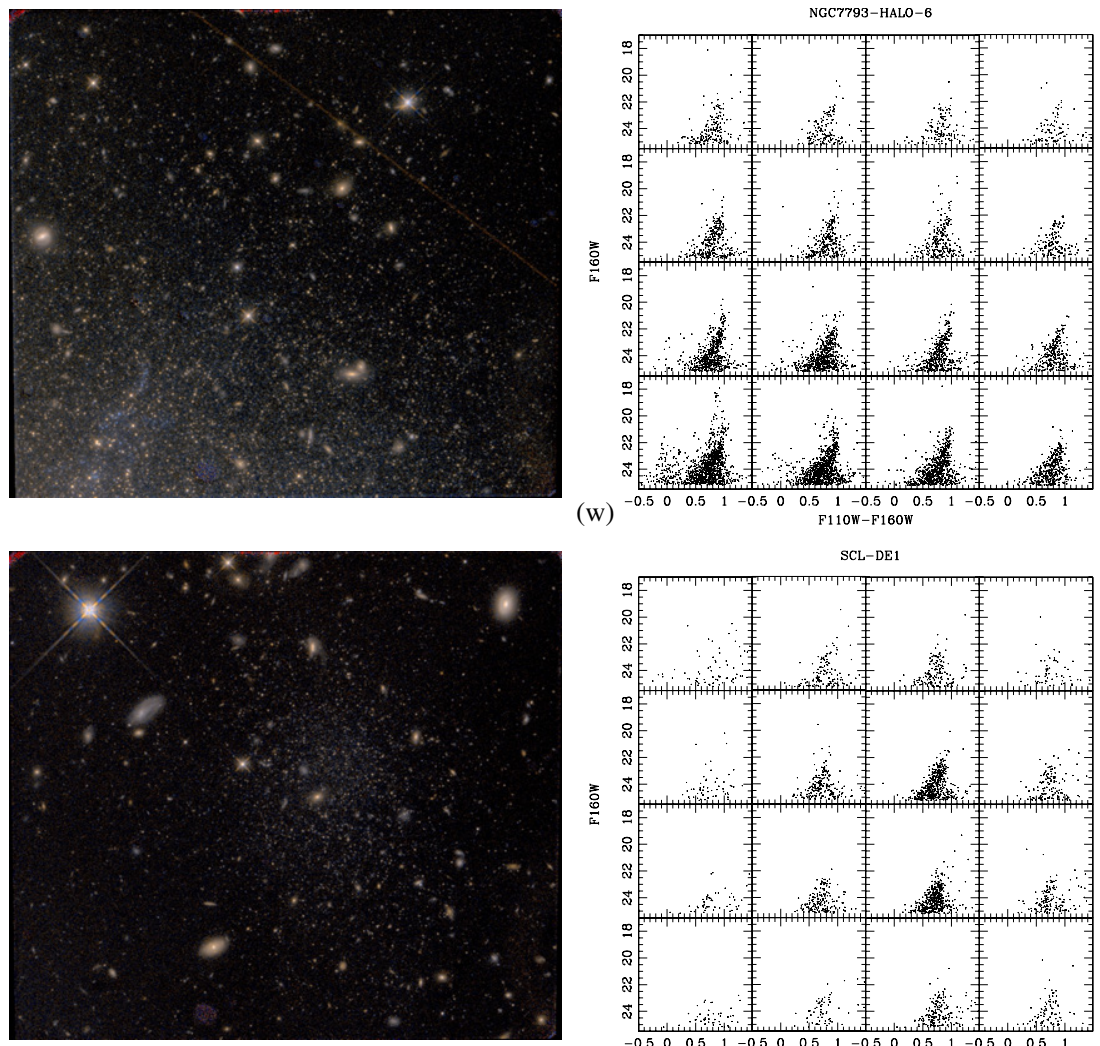

SCL-DE1

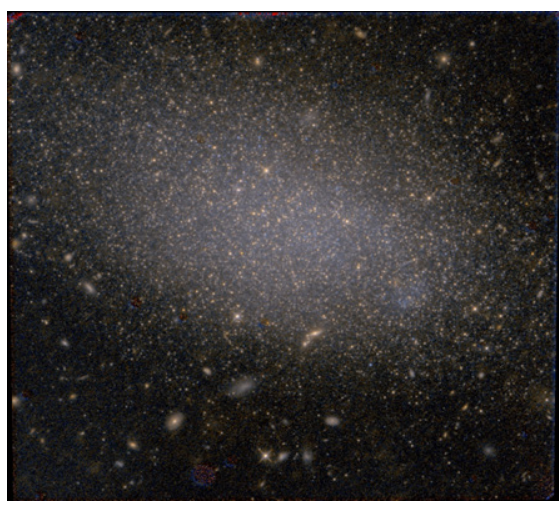

(x)
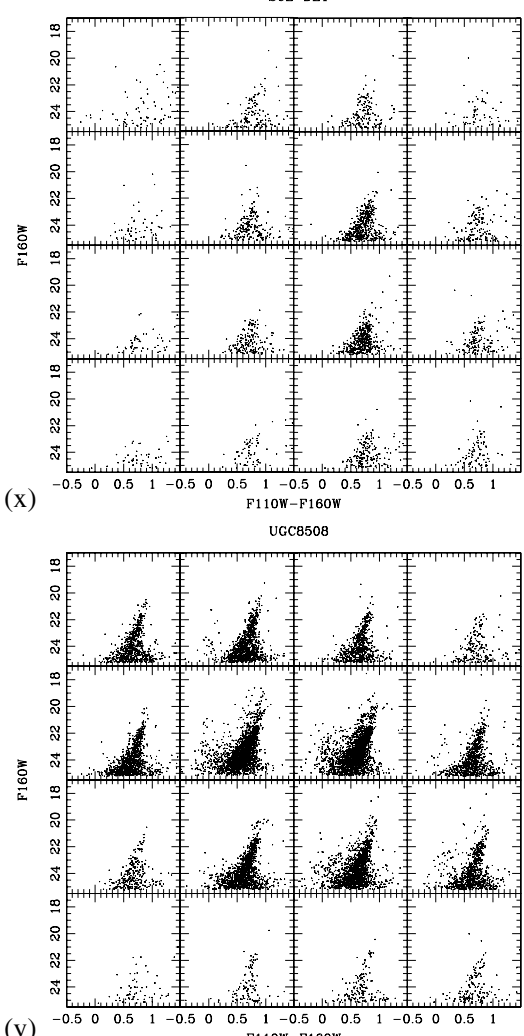

(y)
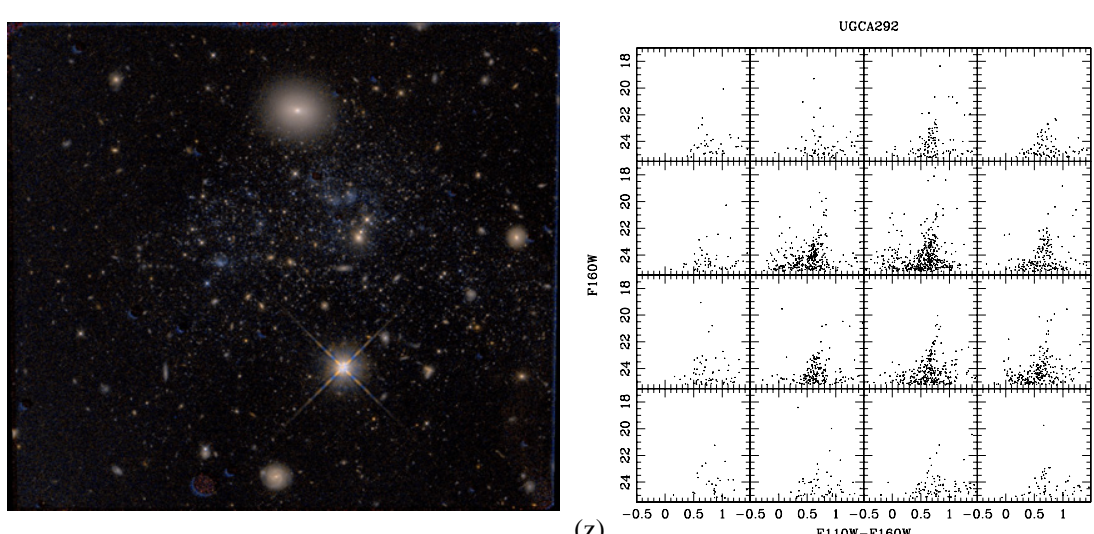

Figure 2. (Continued) 


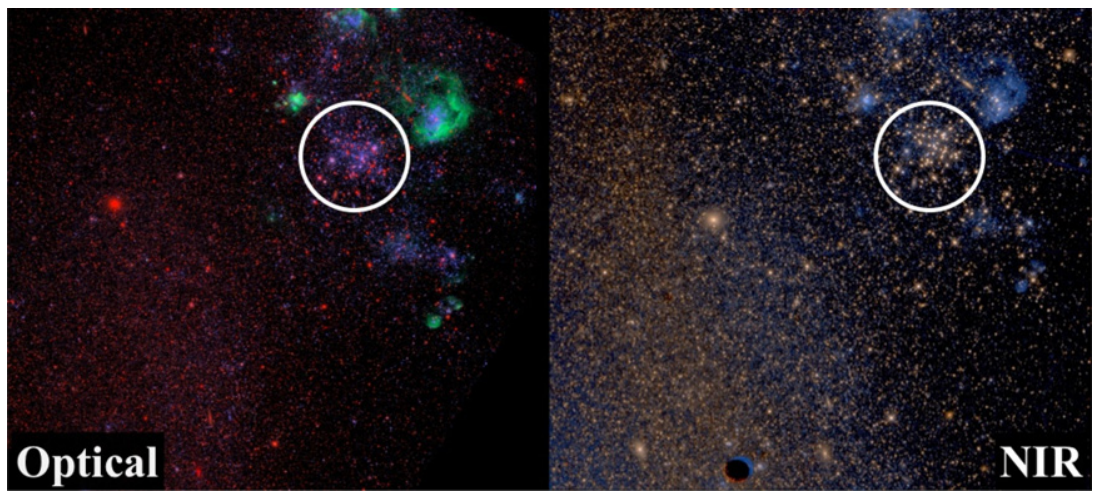

Figure 3. Comparison between a false-color image in the optical (left: $F 435 W+F 555 W+F 814 W$ ) and the NIR (right: $F 110 W+F 160 W$ ) in a star-forming region of I2574. Nebulosity associated with line emission from ionized [S III] and neutral He is visible near $\mathrm{H}$ II regions in the NIR image. The circle indicates a compact star formation region dominated by luminous red core helium-burning (RHeB) stars. This star formation region is old enough that the photoionized HII has recombined $(\gtrsim 10 \mathrm{Myr}$ ), and therefore lacks the diffuse emission seen in the younger neighboring $\mathrm{H}$ II regions. However, it also hosts all of the most NIR luminous sources (see discussion in Section 3.2.2).

(A color version of this figure is available in the online journal.)

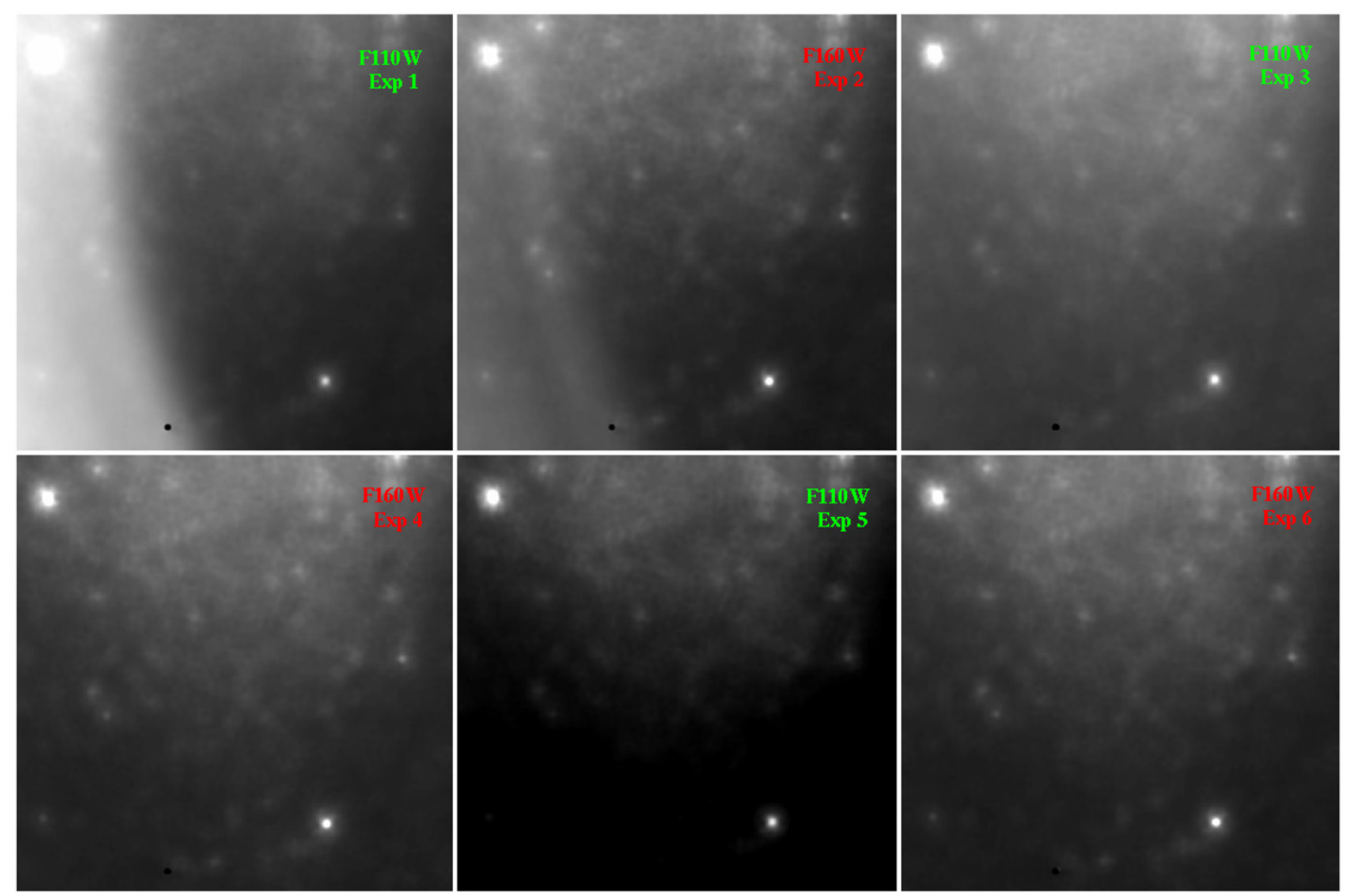

Figure 4. DOLPHOT's estimate of the local sky for a sequence of exposures taken during a single orbit visit for UGC4305-1, based on DOLPHOT's PSF fitting. The first exposure in the orbit is in the upper left, and the final exposure is in the lower right. Exposures alternate between $F 110 \mathrm{~W}$ and $F 160 \mathrm{~W}$, and all images for a single filter have been displayed with a common scale and stretch. There is an overall gradient from top to bottom in all images, due to a larger fraction of unresolved sources in the upper half of the image. There is also a time-dependent feature on the left-hand side of the image, due to scattered light. We show in Figure 5 that this feature appears when the telescope is pointed to within $30^{\circ}$ of a bright Earth limb; the first two exposures in the orbit had average limb angles of $22^{\circ}$ and $26^{\circ}$, respectively, while the third through sixth exposures have limb angles of $31^{\circ}, 34^{\circ}, 36^{\circ}$, and $37^{\circ}$, respectively. Even after the bright limb feature disappears, there are still temporal variations in the overall sky brightness level.

(A color version of this figure is available in the online journal.) 

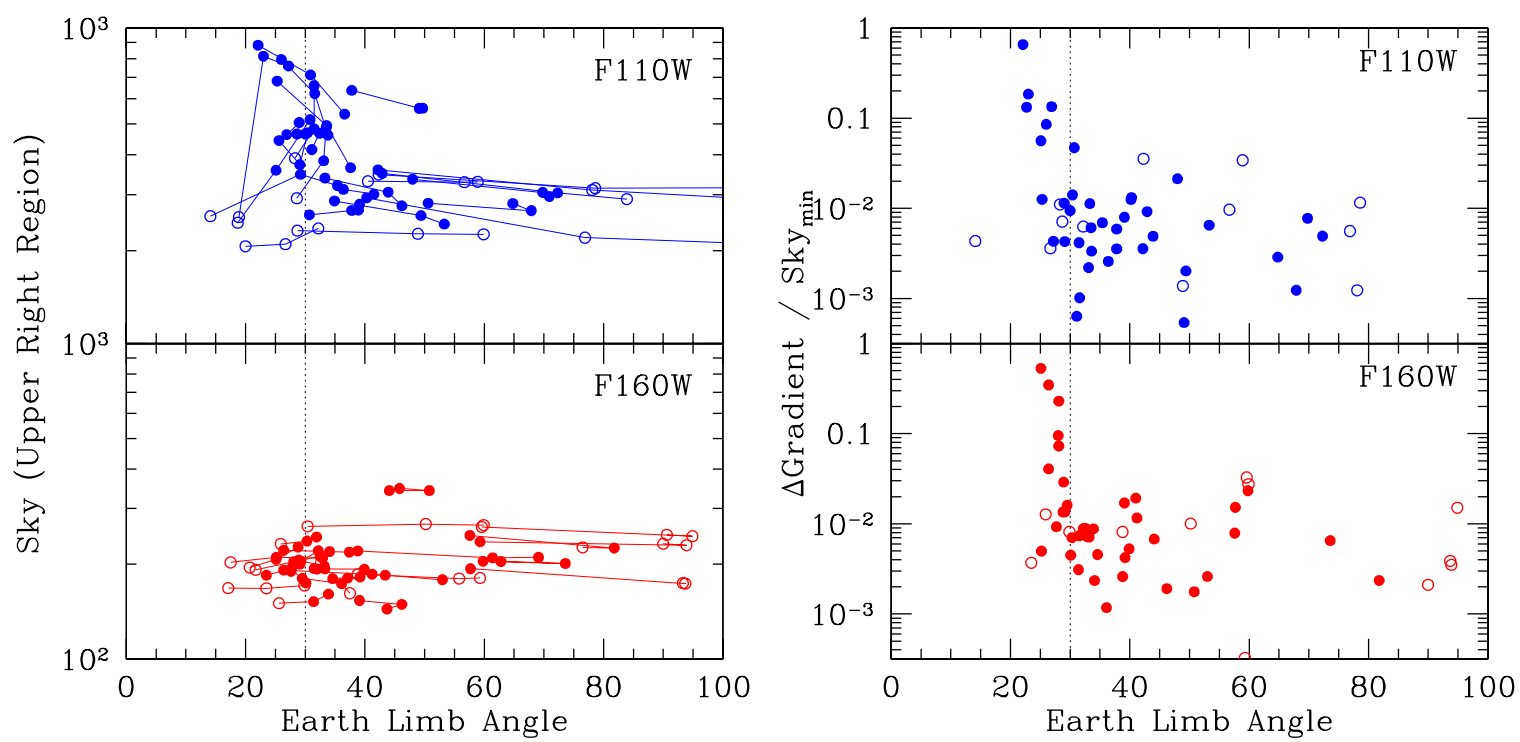

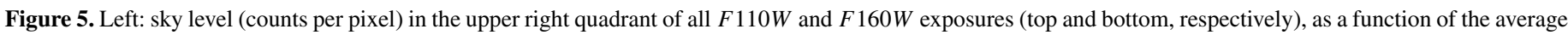

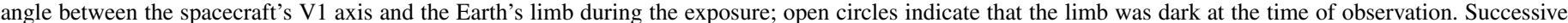

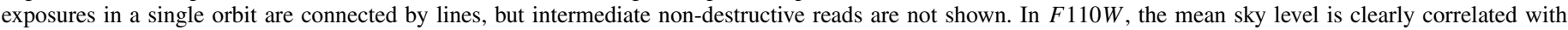

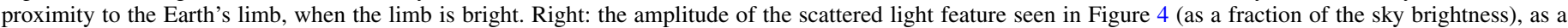

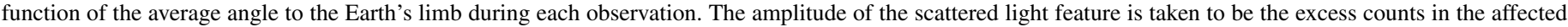

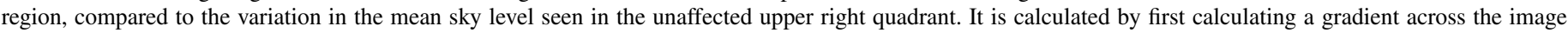

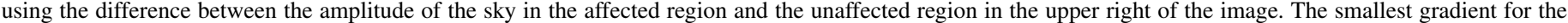

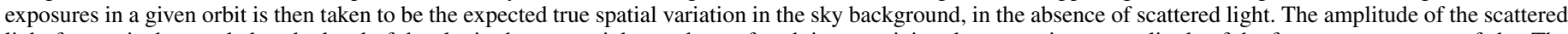

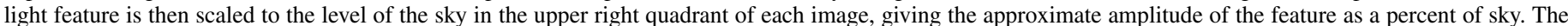
scattered light feature turns on abruptly at Earth limb angles less than $30^{\circ}$.

(A color version of this figure is available in the online journal.)

increased noise levels. For many other possible WFC3/IR programs, however, this may not be the case, and observers should consider specifying a minimum Earth limb angle for their observations.

There are two additional complications that may result from the presence of rapidly varying scattered light, however. The first complication is that some reads affected by scattered light may have pixels that are erroneously flagged as cosmic rays, if flux in the scattered light feature appears as an outlier compared to the large number of unaffected reads; however, we do not see in any obvious increase in the number of masked cosmic rays in the limb brightened regions in Figure 4. The second complication results from the fact that the final IR image is produced by fitting a linear function to a series of non-destructive reads taken during the course of the exposure. However, while the flux in a pixel from a star will rise linearly during the exposure, the flux from the background will not rise linearly when the angle to the limb or the limb's brightness changes during an observation. The assumption of a linear fit may therefore be invalid when the flux in the background is comparable to the flux of the source in a given pixel. One should further note that this effect could be present even when the strong scattered light feature is not obvious, since, as one sees in Figure 5, the uniform sky background can vary strongly during the observation, particularly in $F 110 \mathrm{~W}$. Unfortunately, it is not straightforward for us to evaluate this effect in our data. First, it is difficult to separate the true time-variable sky background, from the spatially variable time-constant background from unresolved stars. Second, we cannot use spatial variations in the CMD to identify systematic errors in the photometry associated with the scattered light feature, since galaxies typically exhibit significant gradients in their stellar populations.

\subsection{Data Reduction and Photometry}

Photometry was performed using the DOLPHOT package ${ }^{15}$ (Dolphin 2000), including a new WFC3-specific module generated in support of this $H S T$ program. The core functionality of DOLPHOT remains as in previous versions (i.e., simultaneous point-spread function (PSF) fitting across a stack of images aligned to sub-pixel accuracy), but the WFC3 update includes several new capabilities: a preprocessing routine that uses the data quality extensions to mask the image and then applies the appropriate WFC3 pixel area maps, a WFC3 PSF library, WFC3 distortion corrections, and new zero points for WFC3. Pixel area maps, distortion terms, zero points, and encircled energy corrections were obtained from the WFC 3 documentation. ${ }^{16}$ The PSF library was computed using the preliminary version of Tiny Tim 7.0 with WFC3 support: ${ }^{17}$ note that the current version of the WFC3/IR Tiny Tim PSF library is based on pre-flight data. For WFC3/IR, PSFs are computed with $10 \times 10$ subsampling and a $3^{\prime \prime}$ radius, for 64 regions on the chip. DOLPHOT also adopts small aperture corrections calculated from uncrowded stars in the image; these corrections were always $\leqslant 0.01 \mathrm{mag}$.

All PSF photometry was performed by minimizing residuals on all individual $\mathrm{flt}$ exposures, and then combined into final magnitudes for each star in each filter. Non-detections are considered to be stars with signal-to-noise ratios $(\mathrm{S} / \mathrm{Ns})$ of less than 4 , for a given filter. Quality parameters are computed and included in the combined photometry as well. Two catalogs were then produced for each field using the DOLPHOT $\mathrm{S} / \mathrm{N}$, sharpness, and crowding parameters. The first "*.st"

\footnotetext{
15 http://purcell.as.arizona.edu/dolphot/

16 WFC3 PSFs from release of 2009 November 15; WFC3 zero points and corrections to infinite aperture from J. Kalirai (ISR 2009-30); WFC3 IDC tables use for distortion correction from t20100519_ir_idc.fits.

17 http://www.stecf.org/instruments/TinyTim/
} 
catalog contains all sources detected in at least one band with $\mathrm{S} / \mathrm{N}>4$, where the sharpness of the source does not exclude the possibility that it is stellar ( sharpness $^{2}<0.1$ ). The second "*.gst" catalog only contains the highest quality photometry (including detections with $\mathrm{S} / \mathrm{N}>4$ in both filters (sharpness $F 110 W+$ sharpness $\left._{F 160 W}\right)^{2}<0.12$ and crowding $_{F 110 W}+$ crowding $\left._{F 160 W}<0.48\right)$. The $*$.gst catalog typically contains $\sim 50 \% \pm 10 \%$ of the sources from the $*$. st catalog. These final parameter cuts were chosen to produce the cleanest CMD features (few stars outside of the known features) without culling large numbers of sources from the features themselves; the culled sources are almost entirely valid stellar detections, but have more uncertain photometry. The number of stars detected varies between 6000 and 40,000 per pointing, with a median of $\sim 10,000$ (Table 2). We also include the maximum and minimum stellar surface density of the *. st detections, calculated within 100 subregions defined by a $10 \times 10$ grid in the image ${ }^{18}$ observations with a higher stellar surface density will be more affected by crowding, and observations with larger differences between the maximum and minimum surface density will have stronger spatial variations in the depth of the CMD and the accuracy of the photometry.

Finally, we assessed the biases in our photometry using artificial star tests. False stars were placed into the image stack 100,000 times, such that the artificial stars sampled the full range of colors, magnitudes, and locations of our real photometry. We recovered the artificial stars using identical photometry routines and post-processing cuts as those applied to the data.

Table 2 includes the resulting " $50 \%$ completeness" magnitude for each filter, as determined by the limit where $50 \%$ of artificial stars inserted into the image are successfully recovered by our photometry (described below) in both filters (as in the $*$.gst catalog). The $50 \%$ completeness limits of our least crowded fields are $\sim 26.2 \mathrm{mag}$ in $F 110 \mathrm{~W}$ and $\sim 25.1 \mathrm{mag}$ in $F 160 \mathrm{~W}$; these limits were significantly fainter than originally expected for photon-limited sources, based on the pre-flight WFC3/IR Exposure Time Calculator. Note, however, that this depth is frequently position dependent, as can be seen from the position-dependent CMDs in the right-hand panels of Figure 2. In crowded regions, the proximity of adjacent stars compromises the recovery of faint stars, producing brighter limiting magnitudes than one would expect based on photon counting statistics alone. The field for SN-NGC 2403-PR has been particularly affected by crowding, and has a $50 \%$ completeness limit that is more than 2 mag brighter than the median of the sample.

Figure 6 shows the magnitude uncertainties reported by DOLPHOT for the $F 110 W$ and $F 160 W$ filters, for the full stellar "*.st" catalog. The small number of points that scatter to larger uncertainties typically come either from regions that were not covered in all three dithered exposures, or that have been flagged as being poorly photometered. The number of stars with higher than average uncertainties are reduced in the culled “*.gst" catalog, which produces somewhat sharper features in the CMD, at the expense of a modest drop in completeness. The effect of this culling can be seen in Figure 7, where we plot the CMDs for the $*$. st and the * . gst photometry catalogs for one of our fields. We will describe the many features in these CMDs in Section 3 below.

In Figure 8 we show the distribution of $F 160 W$ magnitude errors for artificial stars inserted into our images, in bins of

\footnotetext{
18 Empirically, the data in Table 2 indicate that one cannot expect to detect
} more than $\sim 5.5$ stars $\operatorname{arcsec}^{-2}$ in an WFC3/IR frame.
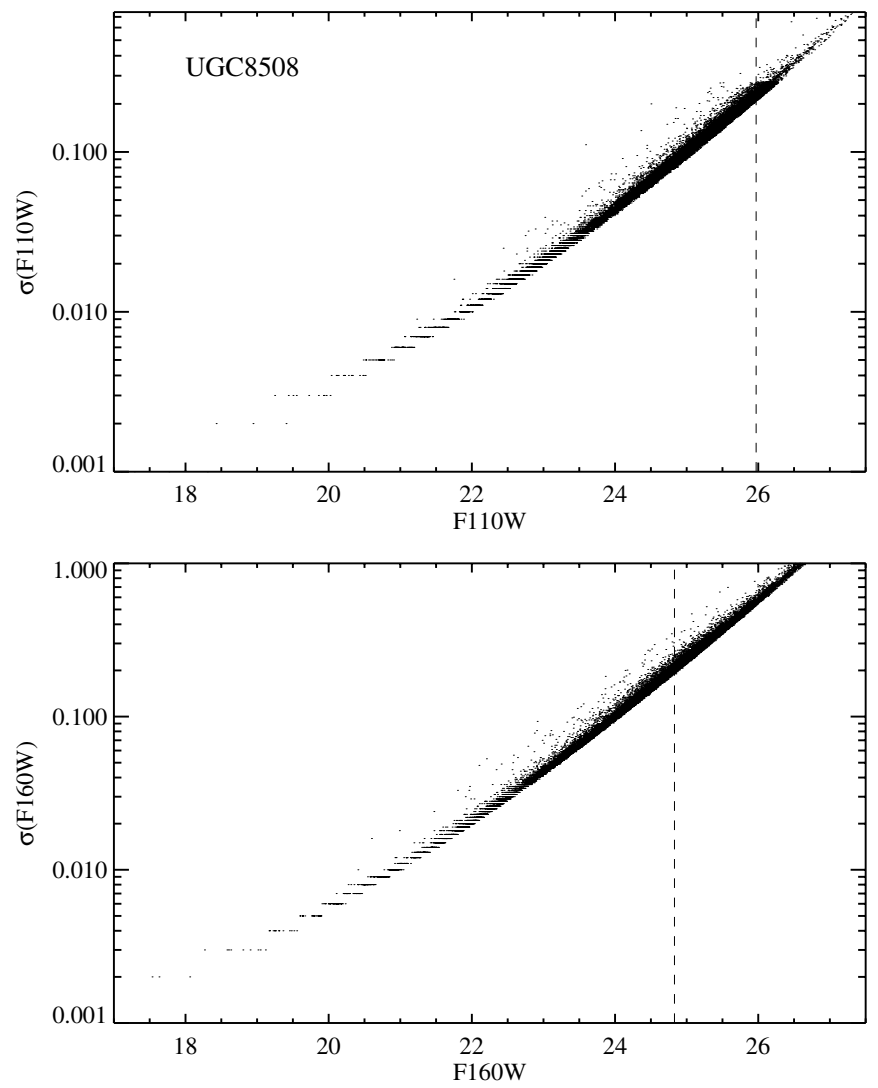

Figure 6. Photometric uncertainties as a function of magnitude in the $F 110 \mathrm{~W}$ (top) and $F 160 \mathrm{~W}$ filters, for a representative field with moderate crowding (UGC8508), using the more complete *.st catalog. The vertical lines indicate the average $50 \%$ completeness level determined from artificial star tests (Table 2). Photometric uncertainties are those reported by DOLPHOT, and do not fully capture the uncertainties due to crowding (see Figure 8).

$1 \mathrm{mag}$, for a crowded and an uncrowded field (left and right plots, respectively). At the extremes, the distributions show a tail toward brighter recovered output magnitudes, due to the blending of faint undetected stars with brighter stars. In the median, however, there is very little bias in the recovered magnitude $(<0.02 \mathrm{mag}$ in the median for the faintest bin, which is nearly an order of magnitude smaller than the photometric uncertainty at the same magnitude). Recovered colors are even less biased, as crowding typically offsets the magnitudes of both filters in similar directions. The amplitudes of these effects are of comparable amplitude in $F 110 \mathrm{~W}$, but are not quite identical due to the different level of crowding and sky brightness in the two filters.

\subsection{Matching NIR and Optical Catalogs}

We match stellar catalogs from the distortion-corrected optical and WFC3 images as follows. We calculate the astrometric transformation by first requiring $\sim 150$ stars that are bright in both the optical and NIR data sets, and that spatially span the entire overlap region between the WFC3 and ACS (or WFPC2) images. We produce a list of candidate alignment stars by first culling the optical and NIR star catalogs from DOLPHOT to only stars that are in the spatial overlap region. We then select all reasonably bright, red stars in each data set (optical color $>0.7$ mag and F814W $<26$ mag; IR color $F 110 \mathrm{~W}-F 160 \mathrm{~W}>0.5 \mathrm{mag}$ and $\mathrm{F} 160 \mathrm{~W}<24 \mathrm{mag}$ ) producing lists of $\sim 1000$ stars in the optical and in the NIR. We sort the resulting two lists by luminosity to preferentially select lumi- 

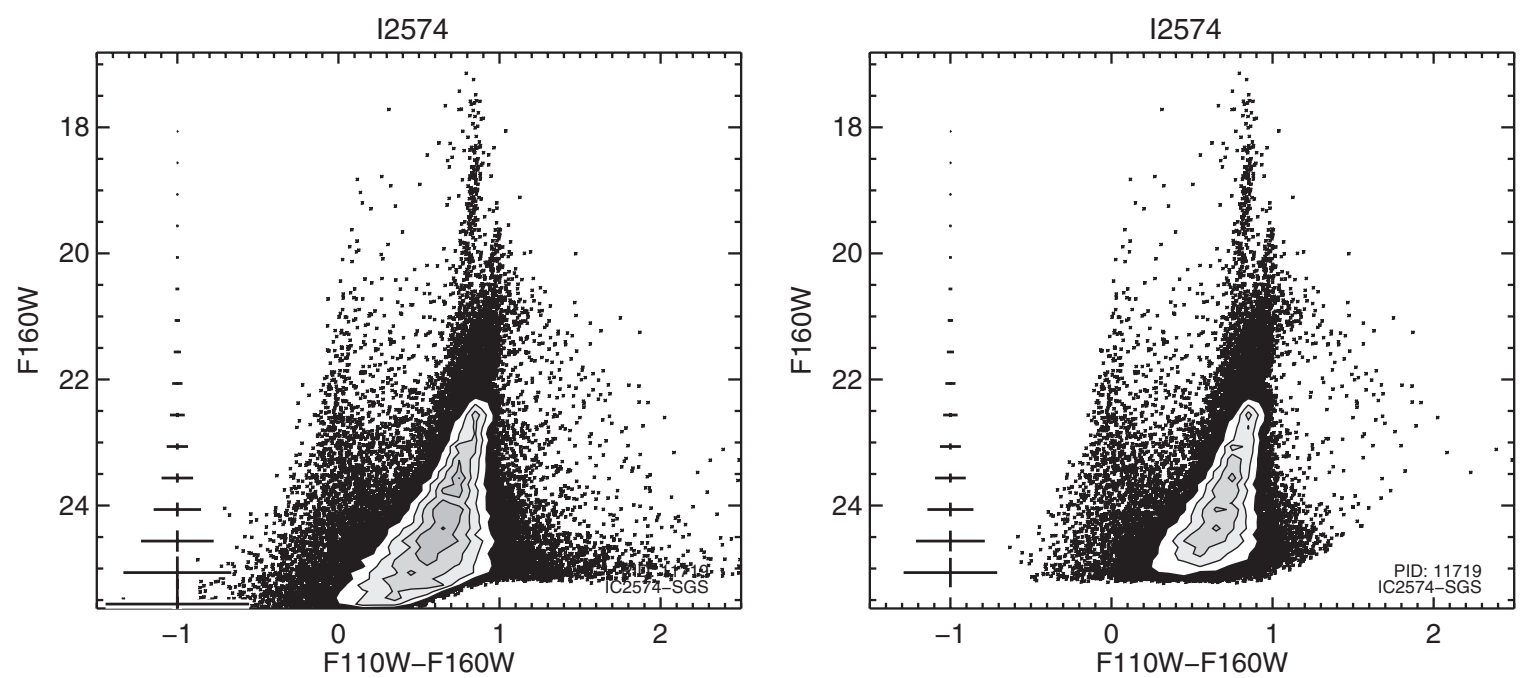

Figure 7. Comparison of the color-magnitude diagrams of the original stellar *.st photometry (left) and the high-quality "cleaned" *.gst photometry (right) for target IC2574-SGS. The original photometry has higher completeness, but at the expense of increased errors due to crowding, producing less sharp features in the CMD. The *. st photometry includes stars that have a signal-to-noise of greater than 4 in only one filter, making the colors unreliable at faint magnitudes; the $*$. gst photometry requires high signal-to-noise in both filters.
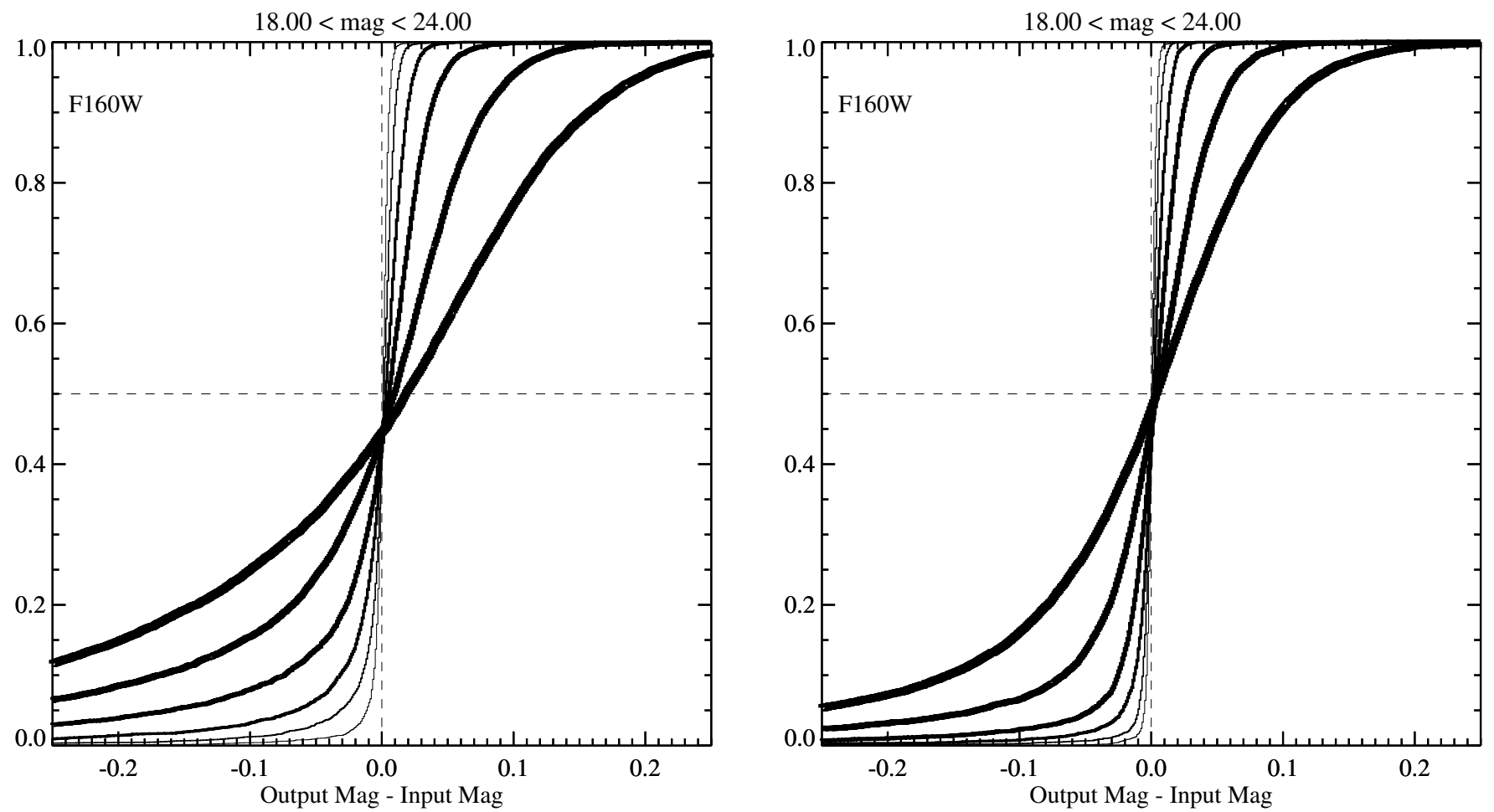

Figure 8. Distribution of differences between the true and the recovered $F 160 \mathrm{~W}$ magnitudes for artificial stars inserted into images of IC2574-SGS (left; highly crowded) and UGC 4459 (right; uncrowded). Distributions are calculated in bins 1 mag wide, with increasing line thicknesses indicating fainter magnitudes; the heaviest line includes stars with $23<m_{F 160 \mathrm{~W}} \leqslant 24$. Crowding biases the photometry very slightly $(<0.004 \mathrm{mag})$ toward fainter observed magnitudes at the median, with larger biases at fainter magnitudes. However, these biases are much smaller than the photometric errors $\left(<0.05 \sigma_{m}\right.$ for IC2574-SGS) in all magnitude ranges. The uncertainties in the magnitude of individual stars are frequently dominated by crowding, however (see Figure 6 for typical photometric errors as a function of magnitude.)

nous stars. Rather than selecting the 150 brightest stars, which are frequently spatially clustered and do not span the full chip, we insist that as long as we have more than one star to choose from, we will select stars with an average separation of $\sim 5^{\prime \prime}$, ensuring broader areal coverage of the alignment stars.

We derive the transformation between the optical and IR star lists by first visually identifying a roughly linear shift between the two coordinate systems. We then iteratively calculate a final transformation using the "method of triangles" described in Valdes et al. (1995), as implemented in the routine $M A T C H^{19}$ by Michael Richmond. First we run a linear fit between the two coordinate systems and then we use that solution as a starting point for a quadratic solution. We find that a cubic solution is generally unnecessary for the transformation between the distortion-corrected WFC3 and ACS images, but we do use it for

\footnotetext{
${ }^{19}$ http://spiff.rit.edu/match/match-0.8/match.html
} 

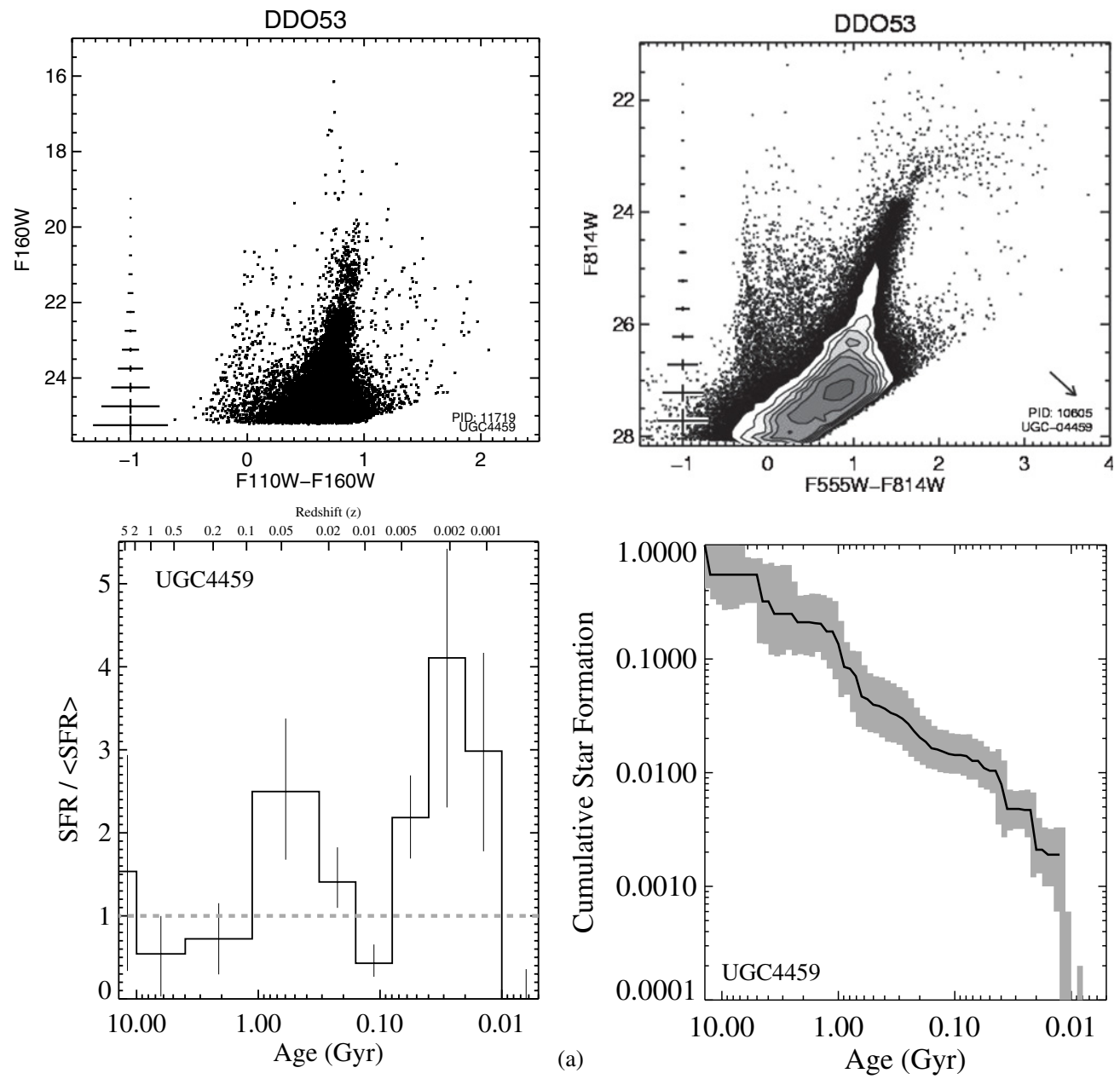

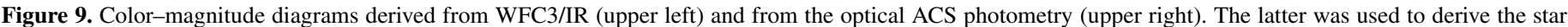

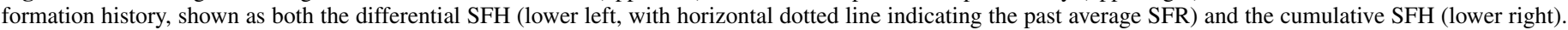

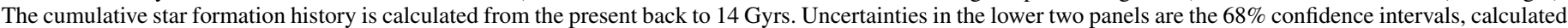

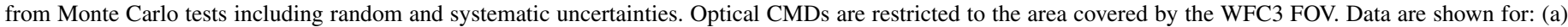

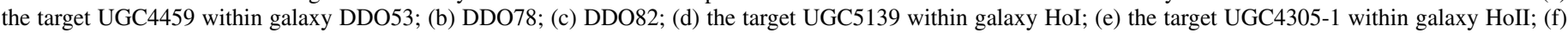

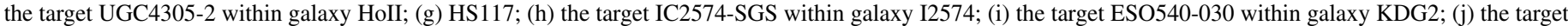

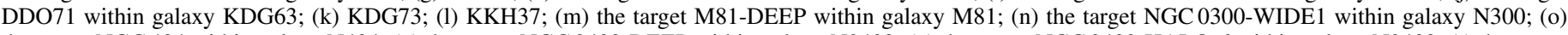

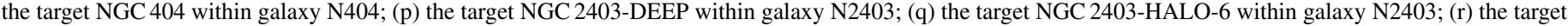

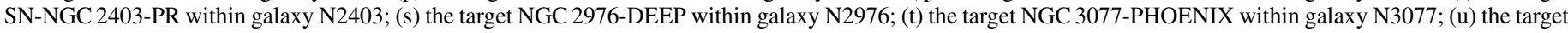

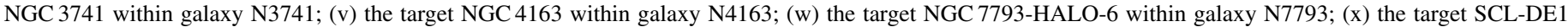
within galaxy Sc22; (y) the target UGC8508 within galaxy U8508; (z) the target UGCA292 within galaxy UA292.

the transformation of the two data sets of WFPC2 observations. We apply the transformation to the entire NIR data set bringing it into the optical coordinate system used for the ANGST and ANGRRR data releases. Note that we make no attempt to tie the images to the global astrometric frame, due to the lack of appropriate astrometric standards in the small WFC3 FOVs.

Once the WFC3/IR catalog has been astrometrically aligned to the optical catalog, we match individual stars in the two catalogs. We consider stars to be a match if the angular separation between the stars is less than 0!07 (i.e., $\sim 0.5 \mathrm{WFC} 3 /$ IR pixel). Typically $90 \%$ of the stars in the NIR catalog are well matched to a star in the optical catalog. Unmatched stars typically fall in the ACS chip gap or in the diffraction spikes of optically saturated bright stars.

\subsection{Characterizing the Age of the Stellar Population}

The morphology of the CMDs that result from our NIR and optical photometry reflects the age and metallicity of the underlying stellar population. To provide an initial constraint of these parameters, we have analyzed the SFH of these galaxies using optical CMD fitting, similar to the procedures described in Williams et al. (2011) and Weisz et al. (2011), but restricted to the optical data that overlap the WFC3/IR FOV.

Specifically, we measured the star formation rate (SFR) and metallicity as a function of stellar age, by fitting the optical CMDs using the software package MATCH (Dolphin 2002). We adopted magnitude cuts set to the $50 \%$ completeness limits, and then fit the CMDs using linear combinations of the stellar evolution models of Girardi et al. (2002, with updates in Marigo et al. 2008 and Girardi et al. 2008), populated with stars following an initial mass function (IMF) with a slope of -2.3 and a binary fraction of 0.35 (with random mass sampling), convolved with the photometric error and completeness statistics derived from artificial star tests. We first fit the data assuming a single foreground reddening and distance, adopting values used in the ANGST survey (Dalcanton et al. 2009) based on the Schlegel et al. (1998) Galactic dust maps and the magnitude of the TRGB, respectively. The best fit provides the relative 

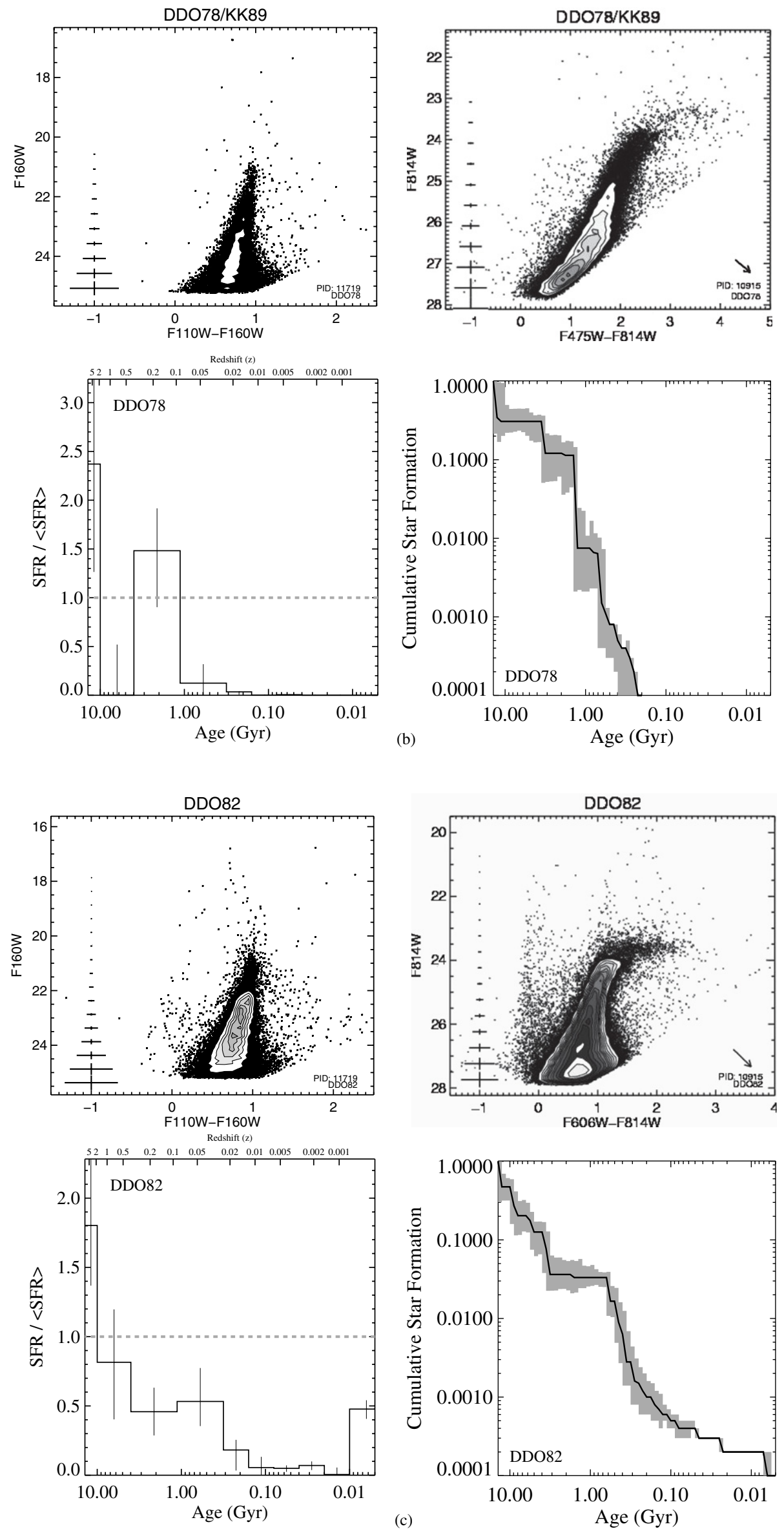

Figure 9. (Continued) 

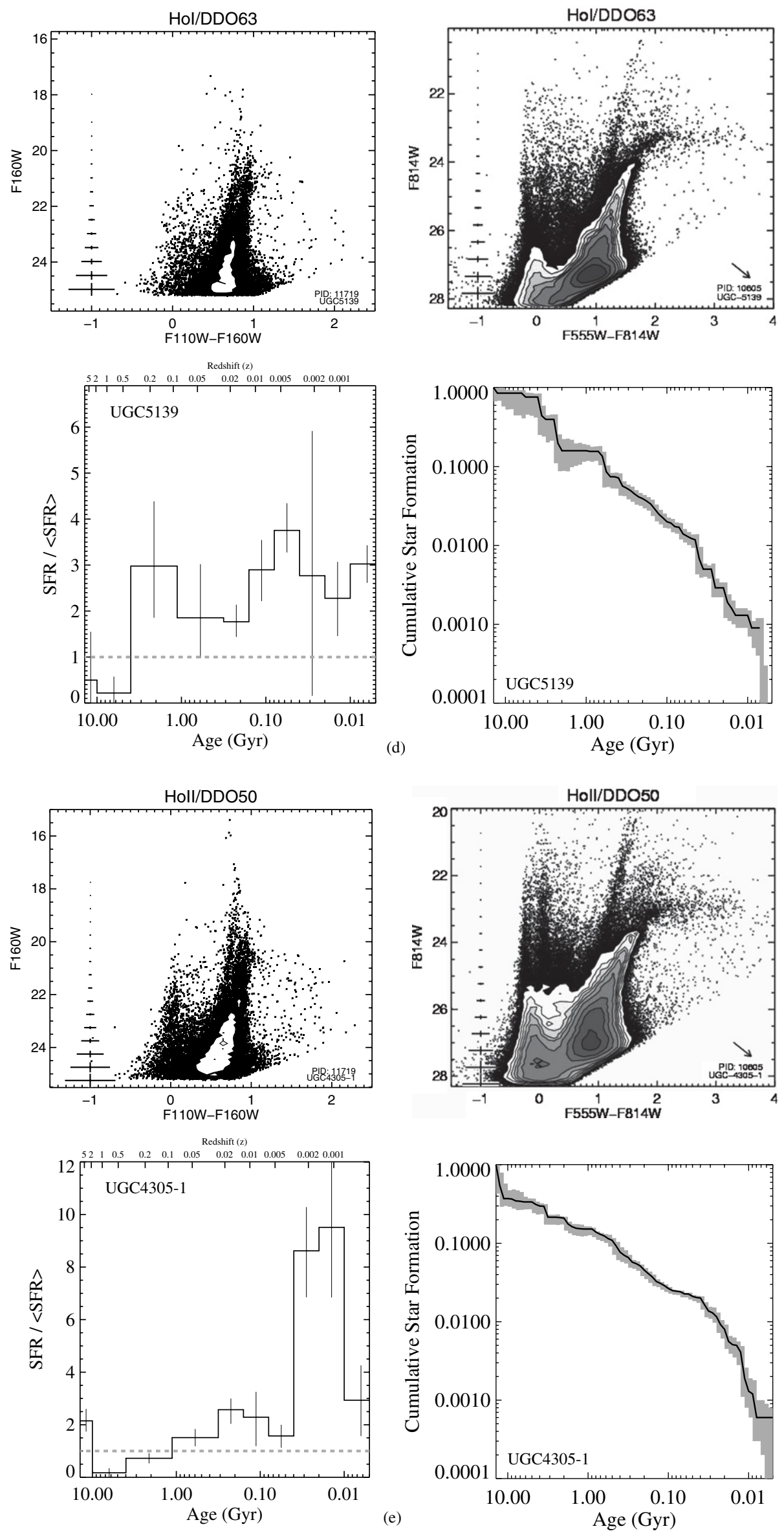

Figure 9. (Continued) 

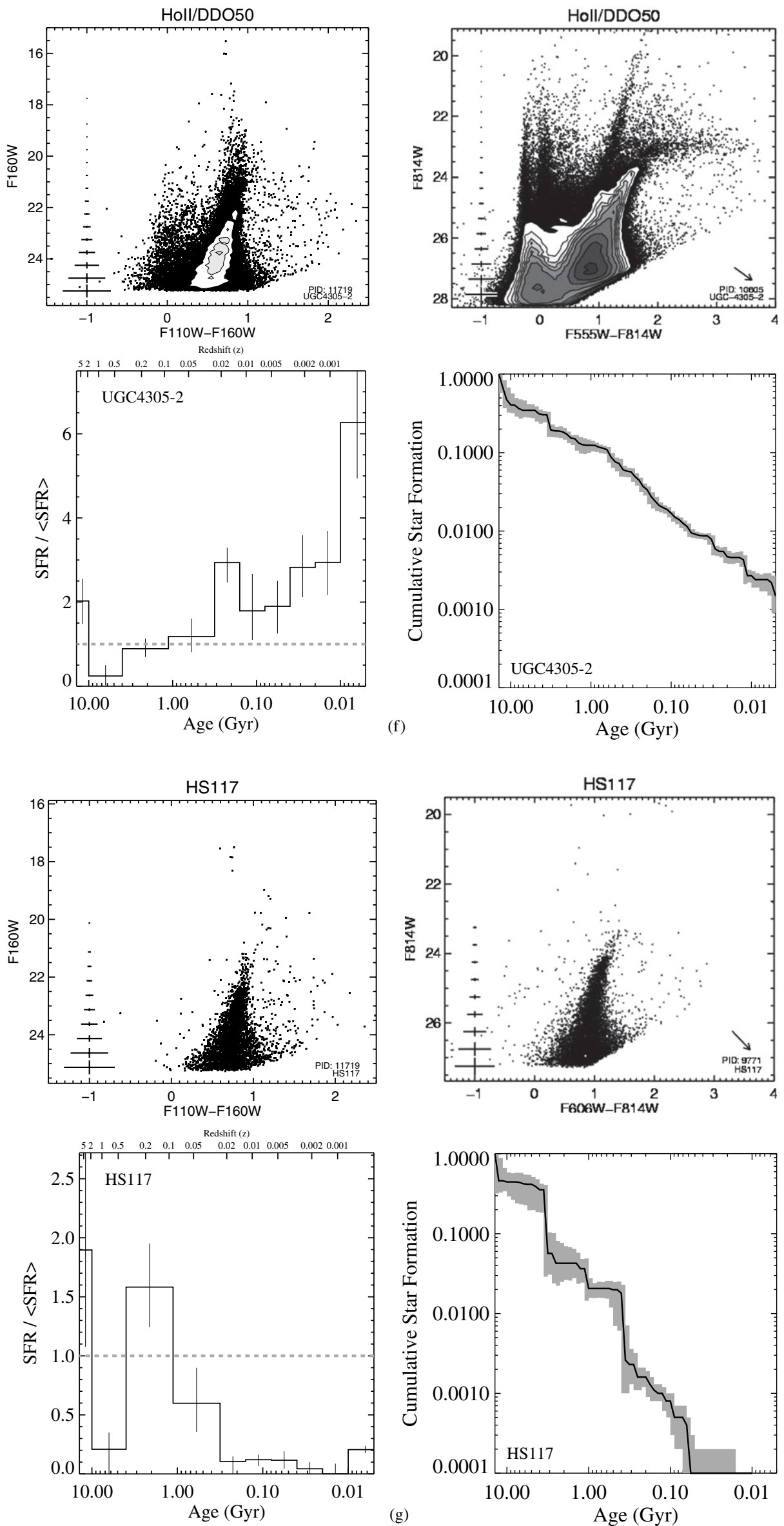

Figure 9. (Continued) 

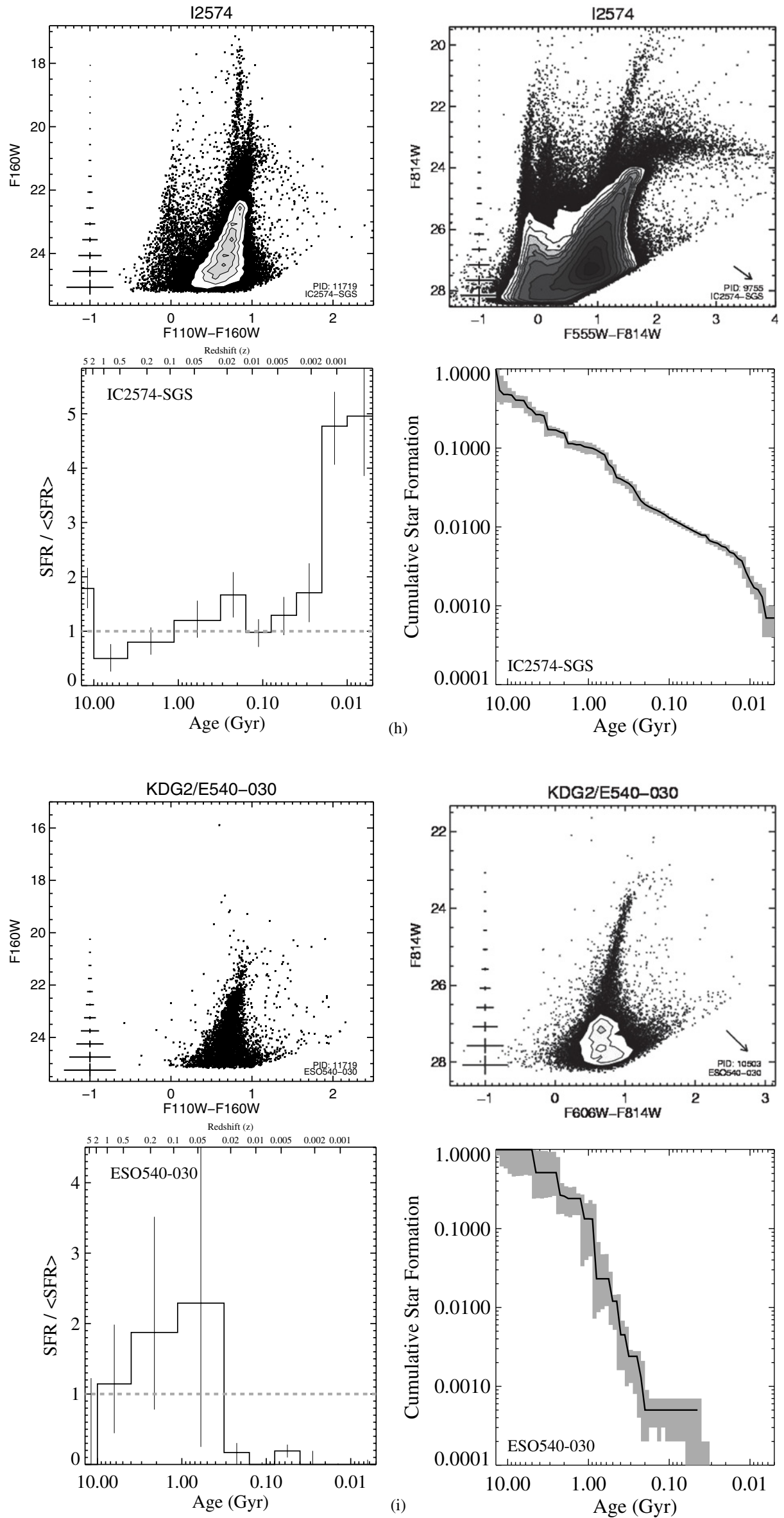

Figure 9. (Continued) 

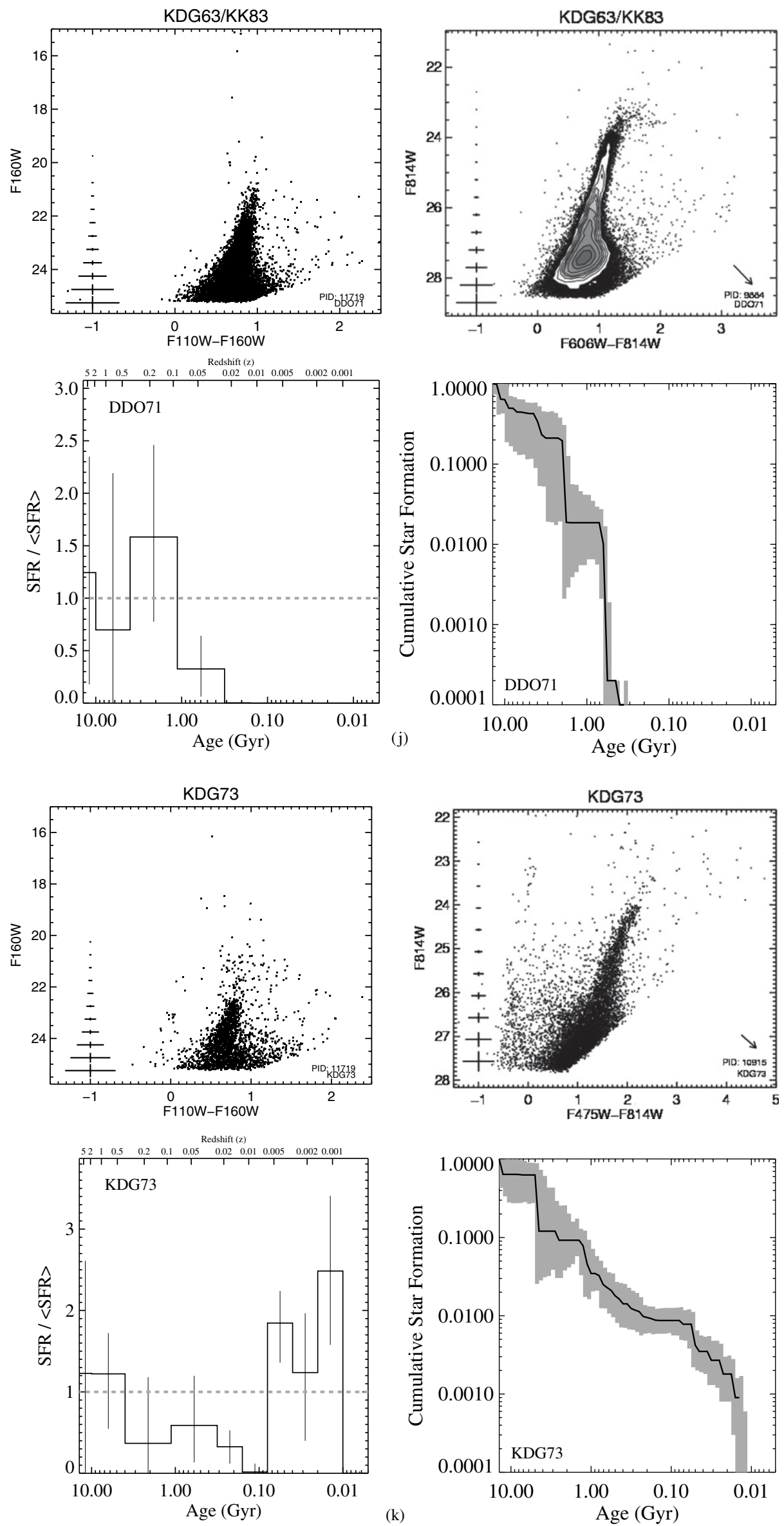

Figure 9. (Continued) 

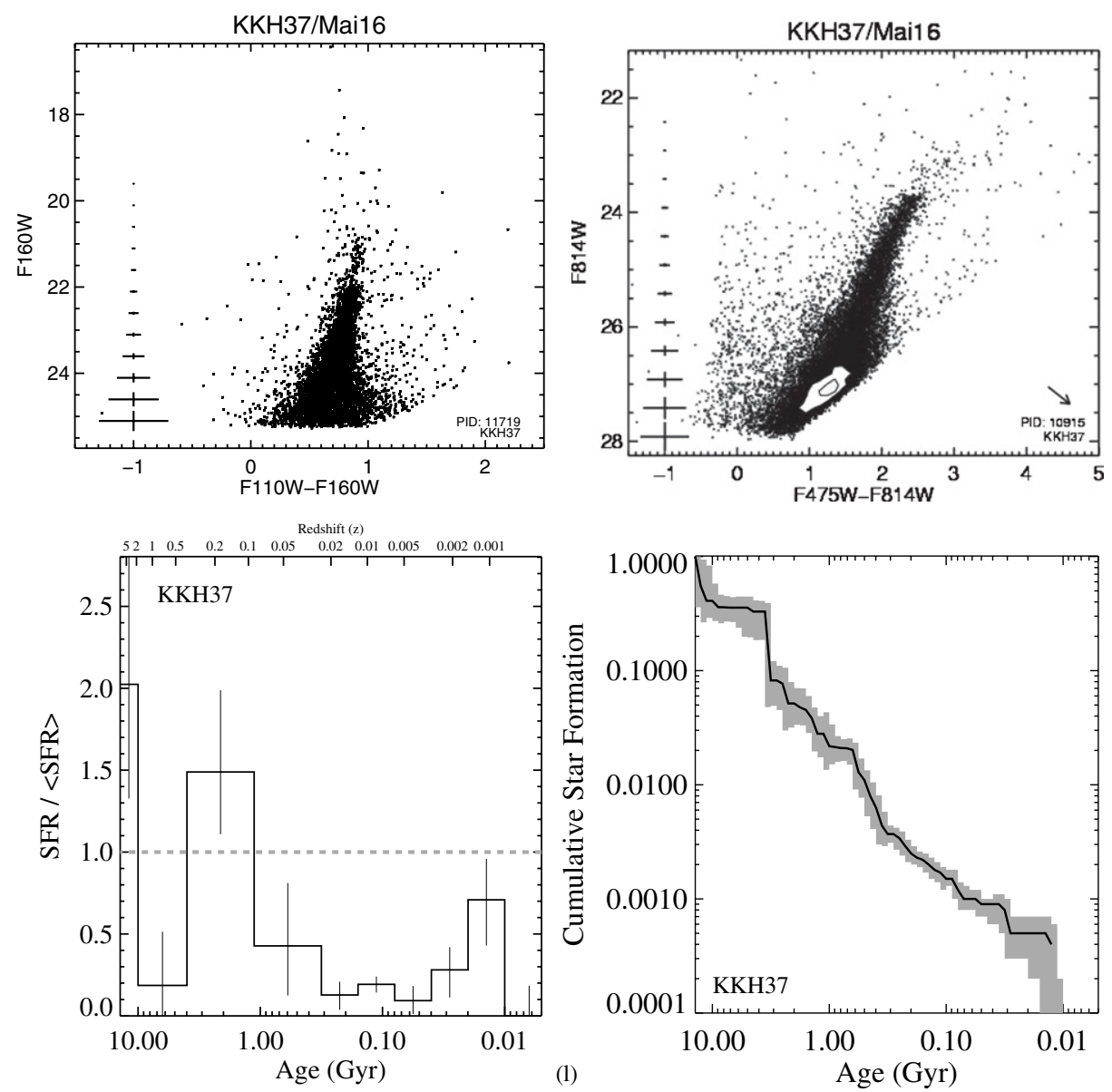

Figure 9. (Continued)

contribution of stars of each age and metallicity in each field, which is then converted into cumulative stellar mass produced as a function of time. For consistency with Weisz et al. (2011), we restricted the metallicity evolution to be constant or increasing with time, for galaxies in common with the Weisz et al. (2011) dwarf sample. This choice provides more accurate recent SFHs, at the expense of introducing occasional biases against star formation in the oldest bin for the few galaxies with very deep data.

To assess the uncertainty of the best fit, we ran extensive Monte Carlo tests. These tests assess two types of uncertainties: (1) random errors due to Poisson sampling of the CMD and errors in photometry and (2) systematic errors due to deficiencies in the stellar evolution models, or due to offsets in distance, reddening, and/or magnitude zero points. The Poisson errors are accounted for by generating artificial CMDs from the bestfit convolved model 100 times, and refitting the resulting CMD. While fitting each of these realizations, the systematic errors are assessed by introducing small random shifts in $\log \left(T_{\text {eff }}\right)$ and $M_{\text {bol }}$. The random values were drawn from a Gaussian distribution with a width of 0.03 in $\log \left(T_{\text {eff }}\right)$ and 0.41 in $M_{\text {bol }}$. The size of these shifts is set by differences between models in the literature, and therefore serves as a proxy of the effects of our particular choice of stellar evolution models. Our final uncertainties are the $68 \%$ confidence intervals of the results of all of our Monte Carlo test fits. These total uncertainties are used as the error bars in all subsequent plots and analysis. Note however that adjacent time bins in the SFH are covariant to some degree, such that when one time bin fluctuates high, the adjacent bins fluctuate low. As a result, the plots of the cumulative SFH offer a truer representation of the uncertainties.

The resulting SFH and cumulative age distributions are shown in the bottom panels of Figure 9. The lower left panel shows the SFR as a function of time, and the lower right-hand panel shows the cumulative $\mathrm{SFH}$, measured from the present back to 14 Gyr ago. A detailed discussion of the SFH analysis of the NIR CMDs will be discussed in an upcoming paper.

\section{COLOR-MAGNITUDE DIAGRAMS}

In this section we describe the properties of stellar populations in the $F 110 W+F 160 W$ filter set using both models (Section 3.1) and observations (Section 3.2). We discuss the qualitative dependence of these properties on the age of the stellar population, as derived from optical CMDs in Section 2.5.

\subsection{Overview of Key Features in Model CMDs}

To elucidate interpretation of the CMDs, in Figure 10 we show simulated NIR CMDs, color-coded either by age or stellar mass (for a constant SFR at a fixed metallicity of $[\mathrm{Fe} / \mathrm{H}]=-1.45$ ), or by metallicity (for a burst of star formation between 8.9 and $11.2 \mathrm{Gyr}$ ). These simulations include the updated models for AGB mass loss at low metallicity described in Girardi et al. (2010), and use the artificial stars from the M81-DEEP 

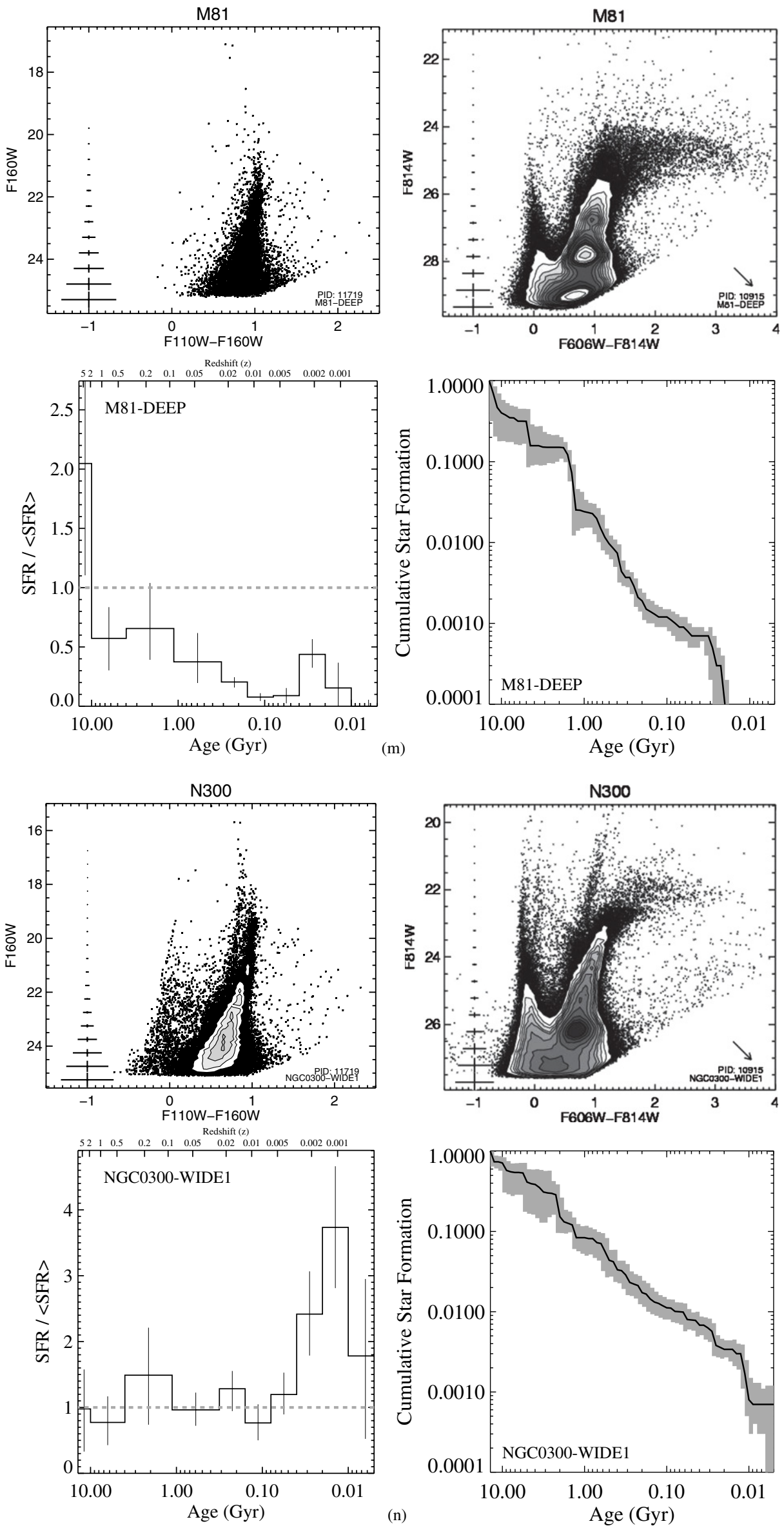

Figure 9. (Continued) 

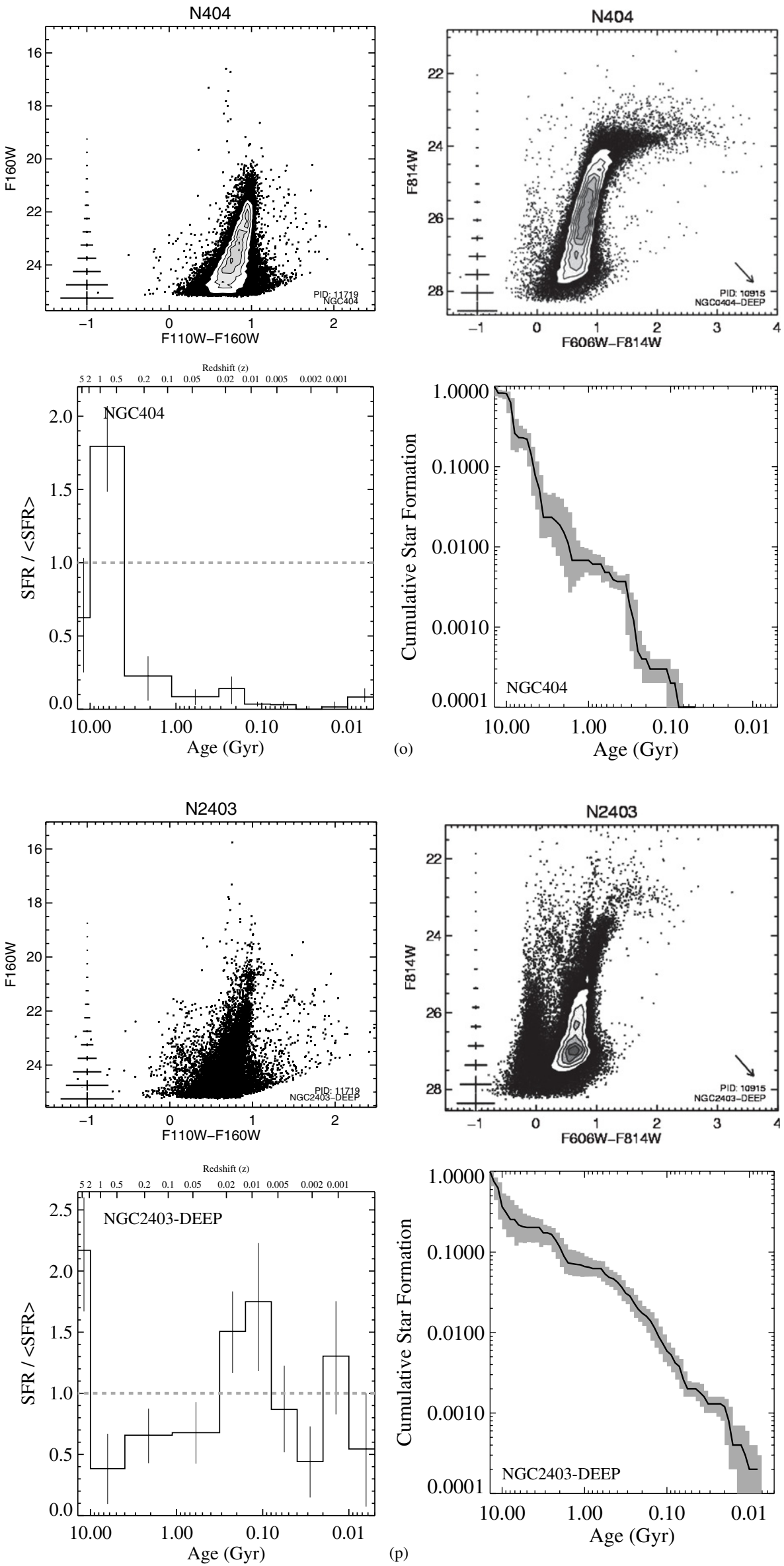

Figure 9. (Continued) 

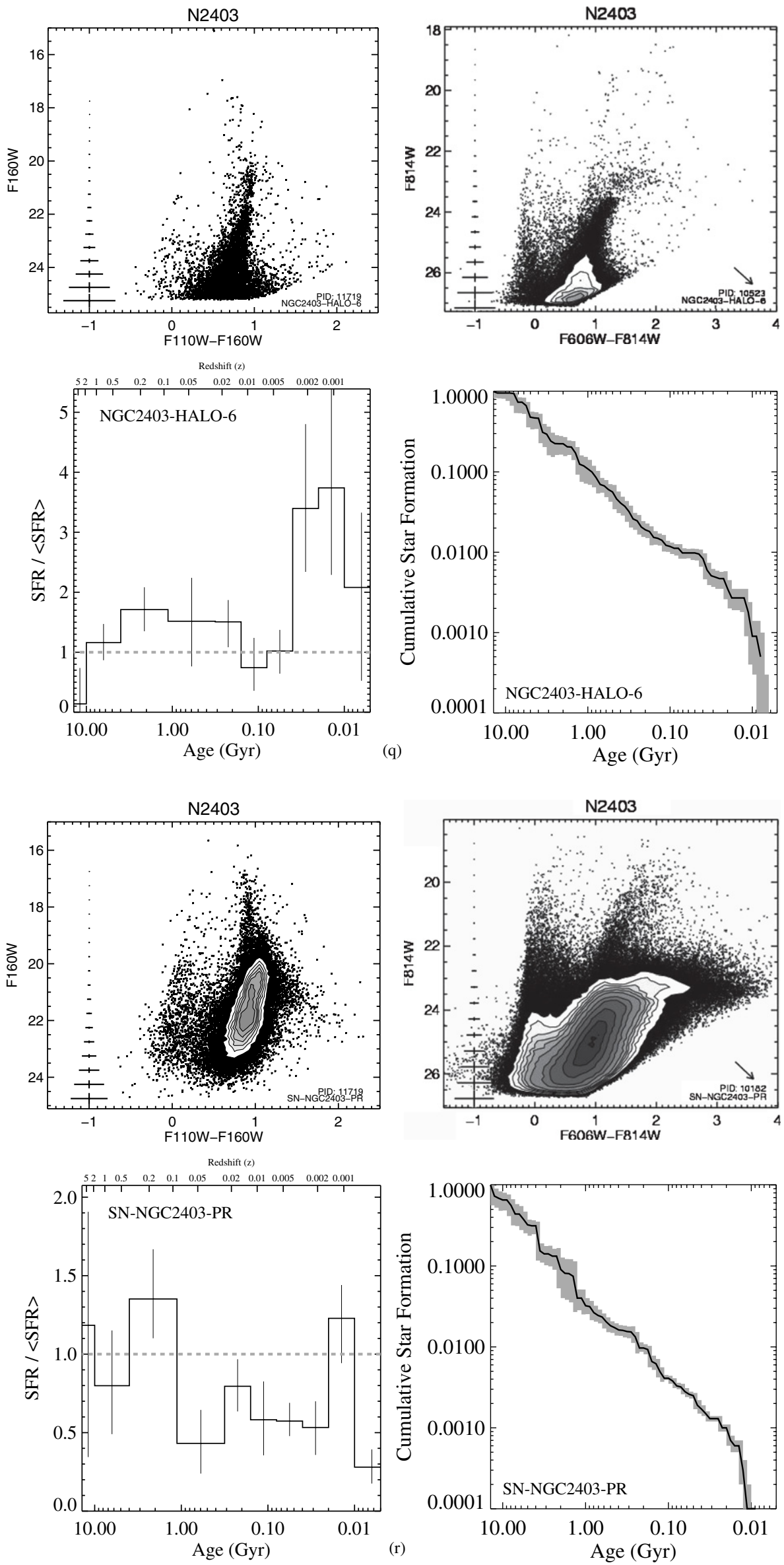

Figure 9. (Continued) 

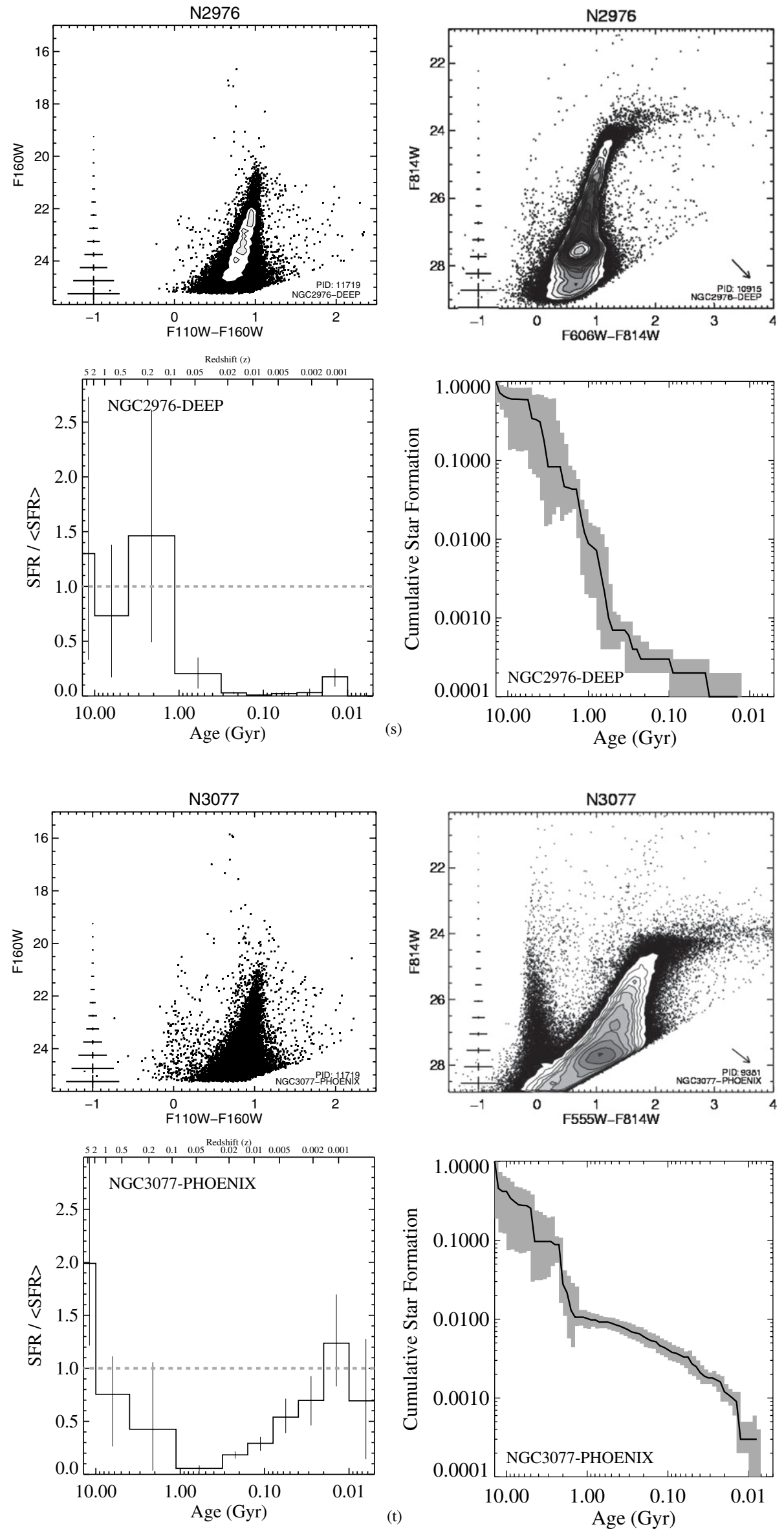

Figure 9. (Continued) 

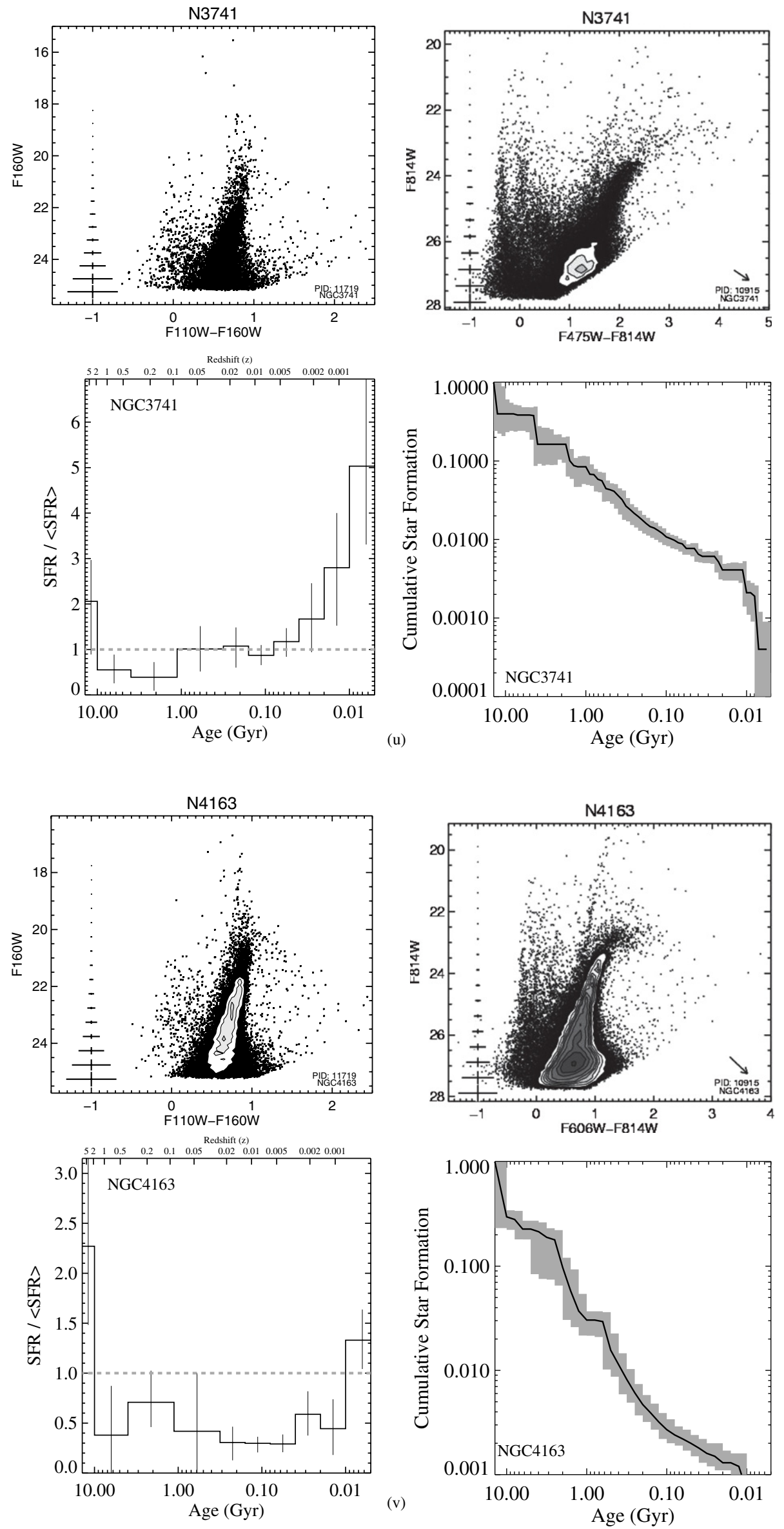

Figure 9. (Continued) 

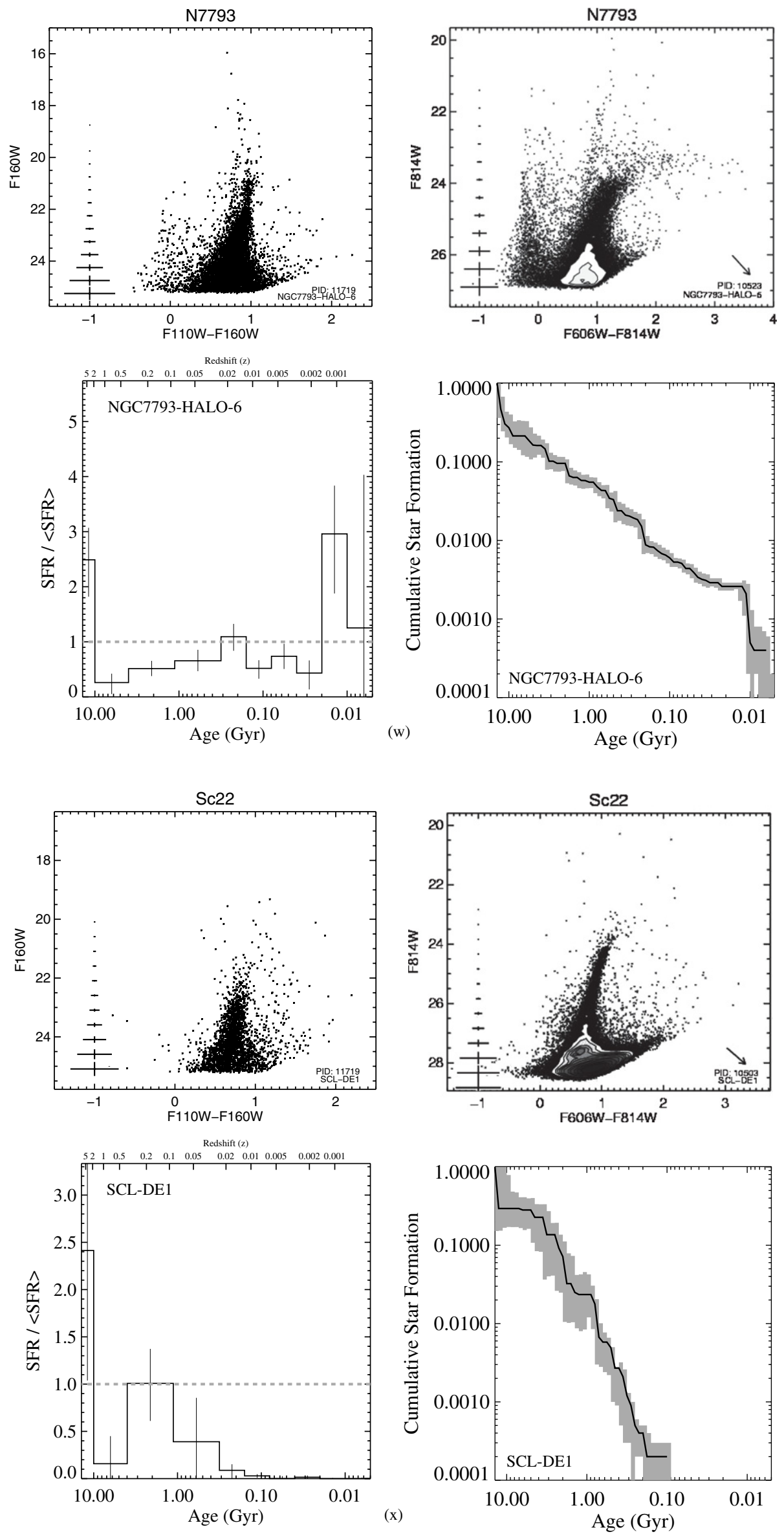

Figure 9. (Continued) 

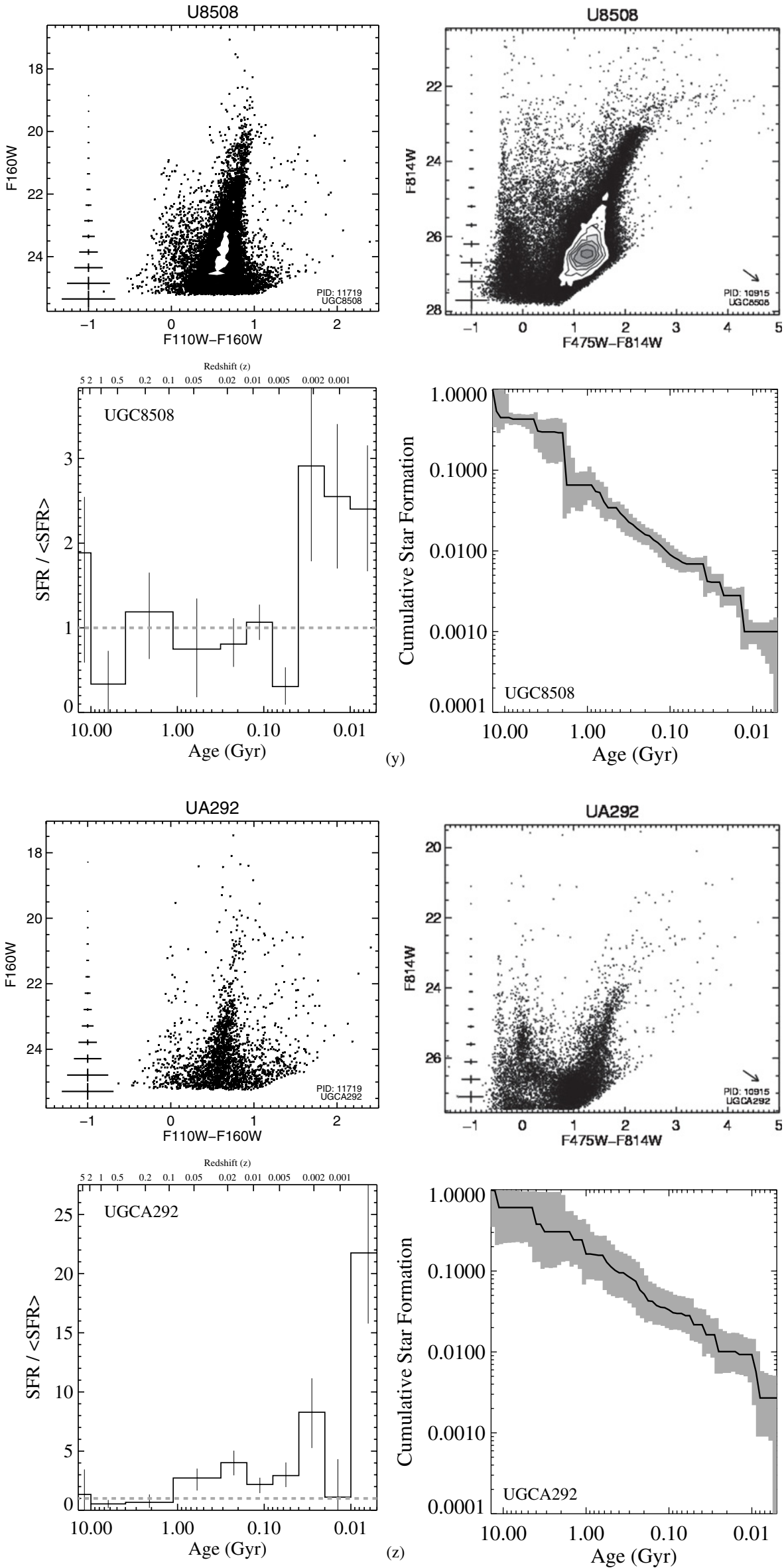

Figure 9. (Continued) 

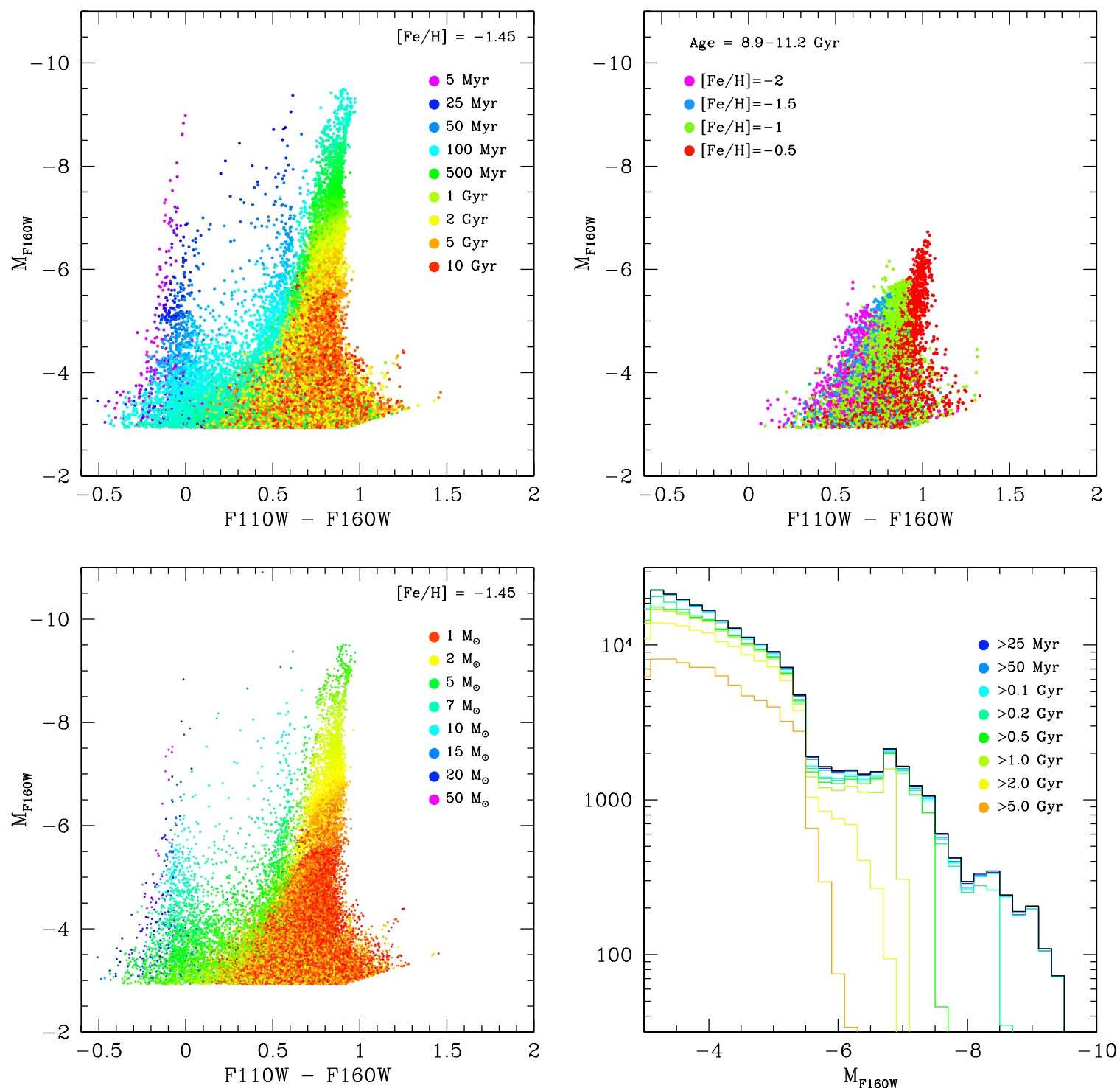

Figure 10. Simulated CMDs showing the location of populations color-coded by age (upper left), initial stellar mass (lower left), and metallicity (upper right). The plots on the left and bottom right assume a constant star formation rate and metallicity of $[\mathrm{Fe} / \mathrm{H}]=-1.45$. The plot on the upper right assumes a constant star formation rate over a narrow age interval $(8.9-11.2 \mathrm{Gyr})$, for a range of metallicities. The plot on the lower right shows the total luminosity function, along with the contributions of stars older than a given stellar age, for stars and ages color-coded as in the CMD on the upper left. Stars younger than 2 Gyr contribute significant numbers of NIR bright stars. Simulations use Padova isochrones with updated AGB models from Girardi et al. (2010), and assume photometric errors based on the artificial star tests for the M81-DEEP field; as we show in Melbourne et al. (2011), the current implementation of these models underestimate the contribution from red core helium-burning stars compared to the data.

(A color version of this figure is available in the online journal.)

field to calculate typical photometric uncertainties and biases. Comparable figures for optical CMDs can be found in Dalcanton et al. (2009).

In both the optical and the NIR, the most prominent feature is the RGB, found at colors of $F 110 W-F 160 W \sim 0.8$ in the NIR and $F 606 W-F 814 W \sim 1$ or $F 475 W-F 814 W \sim 2$ in the optical. This color depends on metallicity (Section 6), and becomes bluer when the metallicity is low (Aaronson et al. 1978). At low metallicities, the optical RGB also becomes more vertical and exhibits less curvature. In the NIR, however, the slope of the RGB is nearly vertical, with only modest variations with metallicity (Ferraro et al. 2000). The ages of stars in the RGB can span a wide range ( $\gtrsim 1 \mathrm{Gyr})$. For the galaxies in our sample, early star formation is particularly vigorous (Weisz et al.
2011; Williams et al. 2011), which will weight the population of RGB stars toward older ages, compared to the constant SFR shown in Figure 10.

One of the most prominent features along the RGB is the "red clump," typically found at 3-4 mag fainter than the TRGB in the optical. The stars in the red clump are burning helium in their cores in the same way as horizontal branch stars, but appear red due to either their young ages (large envelope mass) or their high metallicities (see Girardi \& Salaris 2001; Salaris 2002; Castellani et al. 2000 for theoretical models and Ivanov \& Borissova 2002; Grocholski \& Sarajedini 2002; Valenti et al. 2004 for observational constraints from globular clusters). Unfortunately, our NIR data do not reach this information-rich feature, due to the shortness of our NIR exposures and the 
bright crowding limit resulting from the larger WFC3/IR pixel scale. The absence of this feature reduces the utility of using our NIR CMDs to constrain the relative amounts of ancient and intermediate-age star formation.

Ancient star formation also produces a population of lowmass thermally pulsing AGB stars (TP-AGB; $<1.5 M_{\odot}$ ), found just above the $\mathrm{TRGB}^{20}$ at $M_{F 814 W} \sim-4$. At younger ages, TP-AGB stars are more massive and have much brighter magnitudes (Marigo et al. 2008). As we discuss below in Section 3.2.1, the colors of AGB stars vary little in the NIR, and thus produce nearly vertical sequences for all but the smaller population of red extreme AGB stars (e.g., Nikolaev \& Weinberg 2000; Gullieuszik et al. 2008; Melbourne et al. 2010a; Davidge 2010).

Figure 10 shows that younger stars are expected to dominate the stellar populations blueward and brightward of the RGB. The youngest stars are main-sequence stars, found at the bluest edge of the CMD. While the main sequence is quite well defined in the optical, it is not nearly as distinct in the NIR. This difference must result in part from the fact that only the most luminous high-mass main-sequence stars are detectable in these NIR observations. In the optical CMDs, we typically detect mainsequence stars with masses of $4.5 M_{\odot}$ and above, whereas in the NIR, we can typically only detect main-sequence stars with $\gtrsim 20 M_{\odot}$ (assuming a magnitude limit of $25 \mathrm{mag}$ in $F 160 \mathrm{~W}$ versus 28 mag in $F 814 \mathrm{~W}$, for a median distance of $3.5 \mathrm{Mpc}$ and metallicity of $0.1 Z_{\odot}$ ). Such stars are typically rare, and thus the detectable main sequence will not be sufficiently well populated to appear as a distinct sequence unless there has been ample very recent star formation.

Complicating the clear detection of the main sequence in some cases is the presence of slightly older blue helium core burning $(\mathrm{BHeB})$ stars. These are evolving post-main-sequence 5-20 $M_{\odot}$ stars, with ages of typically 10-500 Myr. These stars, along with their red counterparts (RHeB stars), are formed following the exhaustion of core hydrogen burning, when the core rapidly collapses and the stars' effective temperature decreases. ${ }^{21}$ The core soon reaches an equilibrium, when core helium burning and inner shell hydrogen burning commence, expanding the redder outer layers. A star at this phase is known as a red helium-burning star. As helium in the core is converted into carbon, the RHeBs become visibly hotter and enter the BHeB phase of evolution (e.g., Langer \& Maeder 1995). Although the $\mathrm{BHeB}$ phase occupies a large fraction of the total core helium-burning lifetime (e.g., Bertelli et al. 1994), stars are capable of alternating between BHeB and RHeB stages, with the precise lifetimes determined by the intricate relationships between underlying physical parameters (e.g., Chiosi et al. 1992).

The red and blue core helium-burning sequences are most obvious at optical wavelengths. They appear as two sequences emerging brightward of the red clump, where low-mass $\lesssim 2 M_{\odot}$ core helium-burning stars are found. The BHeB sequence emerges diagonally, heads to bluer colors at higher luminosities, then becomes vertical and parallel to the main sequence at high luminosities and high stellar masses. The RHeB sequence emerges nearly vertically from the red clump, and extends

\footnotetext{
20 AGB stars are also present in the same region occupied by the RGB, but are greatly outnumbered. They cannot be distinguished cleanly as a separate population fainter than the tip of the red giant branch at $M_{F 814 W} \sim-4$. 21 There is some overlap in the literature between the brightest stars in the $\mathrm{HeB}$ sequences and classical red and blue supergiants. However, the former extends to much lower masses and luminosities.
}

steadily brightward, at somewhat bluer optical colors than the RGB. Along these sequences, stars of a given mass appear at a single luminosity, so that the number of stars along the sequences indicate the numbers of evolving stars of different masses. This connection allows the SFR between $\sim 5$ and $\sim 400$ Myr to be essentially read off the sequence, as was pioneered by DohmPalmer et al. (2002), and more recently discussed in McQuinn et al. (2011). More massive, and thus younger, stars appear at very bright magnitudes along both the $\mathrm{BHeB}$ and $\mathrm{RHeB}$ sequences. However, we note that the models for these stars are currently uncertain, and are known not to produce the correct colors or relative number of stars on the red and blue sequences at low metallicities (Dohm-Palmer \& Skillman 2002; McQuinn et al. 2011).

\subsection{Observed Properties of NIR CMDs}

We now turn to the observed NIR and optical CMDs for the fields covered by our WFC3/IR observations, as shown in the top panels of Figure 9. In the upper left panel of Figure 9, we show the NIR CMD of the cleaned *.gst photometry catalogs, uncorrected for foreground extinction or reddening. In the adjacent panel, we show the highest quality optical CMD that is available from overlapping archival imaging. The stars in the optical CMD have been restricted to those that overlap the area covered by the WFC3/IR FOV. These observed CMDs can be compared with the models in Figure 10 to understand which phases of stellar evolution are populating various features in the CMD. We now discuss the principal features of these CMDs.

\subsubsection{Old and Intermediate-age Populations: The AGB and RGB}

In a broad qualitative sense, the NIR CMDs in Figure 9 show the features expected from the stellar models, particularly at old ages. In fields dominated by old stellar populations (as judged from the optical CMD; see KDG 63 or NGC 404 for examples), the NIR CMDs are quite simple. They show roughly 3 mag of a well-defined upper RGB terminating at $M_{F 160 W} \sim-5.7$. The RGB is more vertical and exhibits less curvature than in the optical (see M81-DEEP for an example), as expected from Figure 10. The color at the TRGB varies between 0.7 and 1.1, as we discuss in more detail in Section 4 below. This range of colors agrees well with the range expected from the models in Figure 10. Unfortunately, the data are not deep enough to reveal the red clump in the NIR; this feature is expected to appear $\sim 4$ mag below the TRGB.

Galaxies dominated by old stars also host a sparse population of TP-AGB stars located above the TRGB $\left(M_{F 160 W} \lesssim-5.7\right)$. The NIR population of TP-AGB stars falls in a vertical sequence spanning a narrow range of color, in contrast to the broad "fan" of TP-AGB stars seen in the optical. At optical wavelengths, the TP-AGB stars span a wide range of colors, due to a combination of dust in their circumstellar envelopes, large variations in molecular line spectra with photospheric temperature, the distance between the optical and the NIR peak of an AGB's star bolometric flux (Frogel et al. 1990), and long-period variability (which affects all passbands, but only produces significant color spreads in the optical).

The NIR TP-AGB sequence typically extends $\lesssim 1$ mag in $F 160 W$ above the TRGB. However, the TP-AGB reaches even brighter magnitudes in populations with more recent intermediate-age star formation (see NGC 3741 for an example). The existence of bright, massive TP-AGB stars at younger stellar ages is predicted by the models as well (see Figure 10, and luminosity functions in Olsen et al. 2006). 
At lower luminosities in the TP-AGB sequence, CMDs sometimes show a noticeable "gap" between the brightest RGB stars and the faintest TP-AGB stars above the TRGB (see NGC 2403-HALO-6). This localized drop in the luminosity function seems to have a counterpart in evolutionary tracks of TP-AGB stars (Marigo \& Girardi 2007). The minimum likely reflects the steeper rate of brightening $\left(-d M_{\mathrm{bol}} / d t\right)$ that characterizes the initial stages of the TP-AGB phase, when the first thermal pulses have still not reached the full-amplitude regime and TP-AGB stars are expected to be fainter than predicted by the core mass-luminosity relation. As a result, the brighter late stages of TP-AGB evolution are slower, such that luminosity bins appear relatively more populated than the fainter early stages. Simulated CMDs based on the Marigo \& Girardi (2007) TP-AGB models do show a local minimum in the luminosity function right above the TRGB, as expected. However, the gap is not apparent in the more recent models described in Girardi et al. (2010; lower right panel of Figure 10). The observation of this features opens the possibility that it could help to calibrate the models at the early stages of the TP-AGB.

The mean color at the base of the TP-AGB sequence is comparable to the mean color at the TRGB, such that the TP-AGB sequence appears to emerge vertically from the RGB. However, the cumulative distributions of colors immediately above and below the $\mathrm{TRGB}^{22}$ suggest that the AGB is slightly redder by $0.02-0.04 \mathrm{mag}$ for galaxies where there is minimal contamination from RHeB stars (e.g., KKH37, HS117, DDO82, DDO78, N2976). Visually, the TP-AGB sequence appears to span a smaller range of color than the RGB in some cases. However, there is no statistical evidence that this is the case, as both the RGB and TP-AGB have statistically indistinguishable widths, when the fewer number of stars in the AGB is taken into account.

We note that our sample does not immediately show a large obvious population of carbon-rich AGB stars. In the corresponding $J+H$ ground-based NIR filter set, such stars dominate a roughly diagonal sequence that starts near $M_{\mathrm{H}} \sim-7$ and extends to redder colors and fainter magnitudes. To illustrate this sequence, we show empirical data in Figure 11, using $J-H$ versus $H$ CMD for Two Micron All Sky Survey (2MASS) observations of the LMC. This filter combination is the closest match to the $F 110 W+F 160 W$ filter set used in our observations. ${ }^{23}$ In the LMC data the sequence of carbon-rich and/or extreme AGB stars is roughly horizontal at $M_{\mathrm{H}} \sim-7.3$, and extends redward from the sequence of more numerous oxygen-rich AGB stars. There are a few cases where a comparable red sequence of extreme AGB stars is hinted at in our data (e.g., UGC4305, NGC 4163, NGC 2403-HALO-6, NGC 0300-WIDE1, DDO82). However, in none of these is the sequence as noticeable as in the LMC.

The absence of a strong carbon star tail is initially surprising given that our sample of galaxies is dominated by lower metallicity galaxies than the LMC. The dredge-up of carbon is believed to be more efficient at lower metallicities, both because of more favorable conditions for the occurrence of strong helium shell flashes (Karakas et al. 2002; Stancliffe 2006), and because of the lesser carbon dredge-up needed to

\footnotetext{
22 We define the RGB stars "below" the TRGB as being the 0.25 mag interval starting 0.05 mag fainter than the TRGB, and the AGB stars "above" the TRGB as being the 0.5 mag interval starting 0.05 mag brighter than the TRGB. This selection produces roughly equal numbers of stars in each subsample. 23 For reference, at $J-H=1$, magnitudes in $F 160 \mathrm{~W}$ are 0.28 mag fainter than in $H$, and colors in $F 110 W-F 160 W$ are 0.08 mag redder than in $J-H$, as shown below in Equations (2) and (3).
}

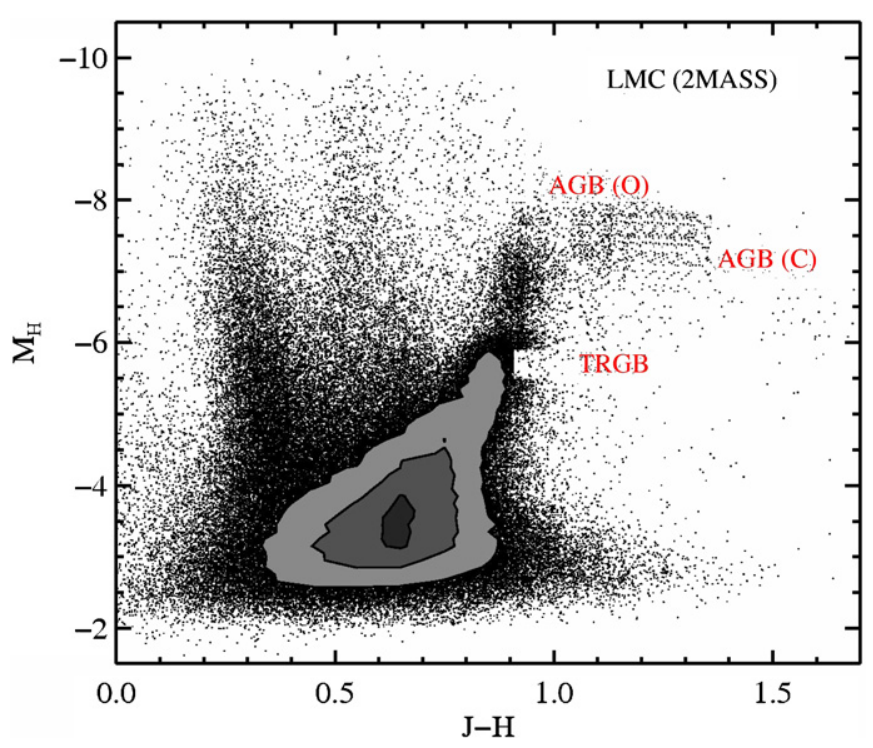

Figure 11. $J-H$ vs. $M_{H}$ 2MASS CMD for stars in the LMC. The sequence extending up from the TRGB at $M_{H}=-6$ is dominated by oxygen-rich AGB stars. The horizontal sequence at $M_{H} \sim-7.3$ extending redward of $J-H \sim 0.9$ contains mostly carbon-rich AGB stars. The main sequence is the bluest vertical sequence at $J-H \sim 0.25$. The vertical sequence at $J-H \sim 0.5$ is dominated by MW foreground stars.

(A color version of this figure is available in the online journal.)

reach a carbon-rich condition (with $\mathrm{C} / \mathrm{O}>1$ at the surface). Thus, one expects carbon-rich stars to be more numerous in low-metallicity galaxies. Empirically, this trend has been seen in several studies (e.g., Battinelli \& Demers 2005; Groenewegen 1999, 2007; Boyer et al. 2011).

On the other hand, carbon-rich stars of low metallicity are significantly hotter than at solar metallicities, which inhibits the formation of the molecules that cause the spectral features typical of carbon stars (Marigo \& Girardi 2007). The resulting reduced opacity in low-metallicity carbon-rich stars (e.g., Marigo \& Aringer 2009) may move the carbon stars back to bluer NIR colors than their high-metallicity counterparts (see, for instance, Figure 7 of Marigo \& Girardi 2007). Thus, while carbon stars may be more numerous in low-metallicity systems, they may be less obvious outliers in NIR CMDs. The difficulty of identifying carbon stars in the NIR has been demonstrated empirically by Battinelli \& Demers (2009) for galaxies within the Local Group.

We believe that the lack of obvious carbon stars is further exacerbated by use of the $F 110 W+F 160 W$ filter set. Simulations with TRILEGAL (Girardi et al. 2005; Girardi \& Marigo 2007) shown in Figure 12 indicate that the majority of carbon-rich AGB stars occupy similar locations as oxygen-rich AGB stars in the WFC3/IR CMDs. These simulations were performed using both the Loidl et al. (2001; top row) and Aringer et al. (2009; bottom row) synthetic spectra for C stars. Whereas carbon stars are distinctly redder in $J-H$ than the oxygen-rich AGB (left column), in $F 110 W-F 160 W$ they are (if anything) slightly bluer (middle column), particularly for the Aringer et al. (2009) atmosphere models. The properties of the carbon-rich AGB population in our sample will be explored in more detail in an upcoming paper.

\subsubsection{Young Populations: The Importance of Red Core Helium-burning Stars}

In contrast to older stellar populations, young stellar populations have surprisingly complex NIR CMDs. Fields with the 

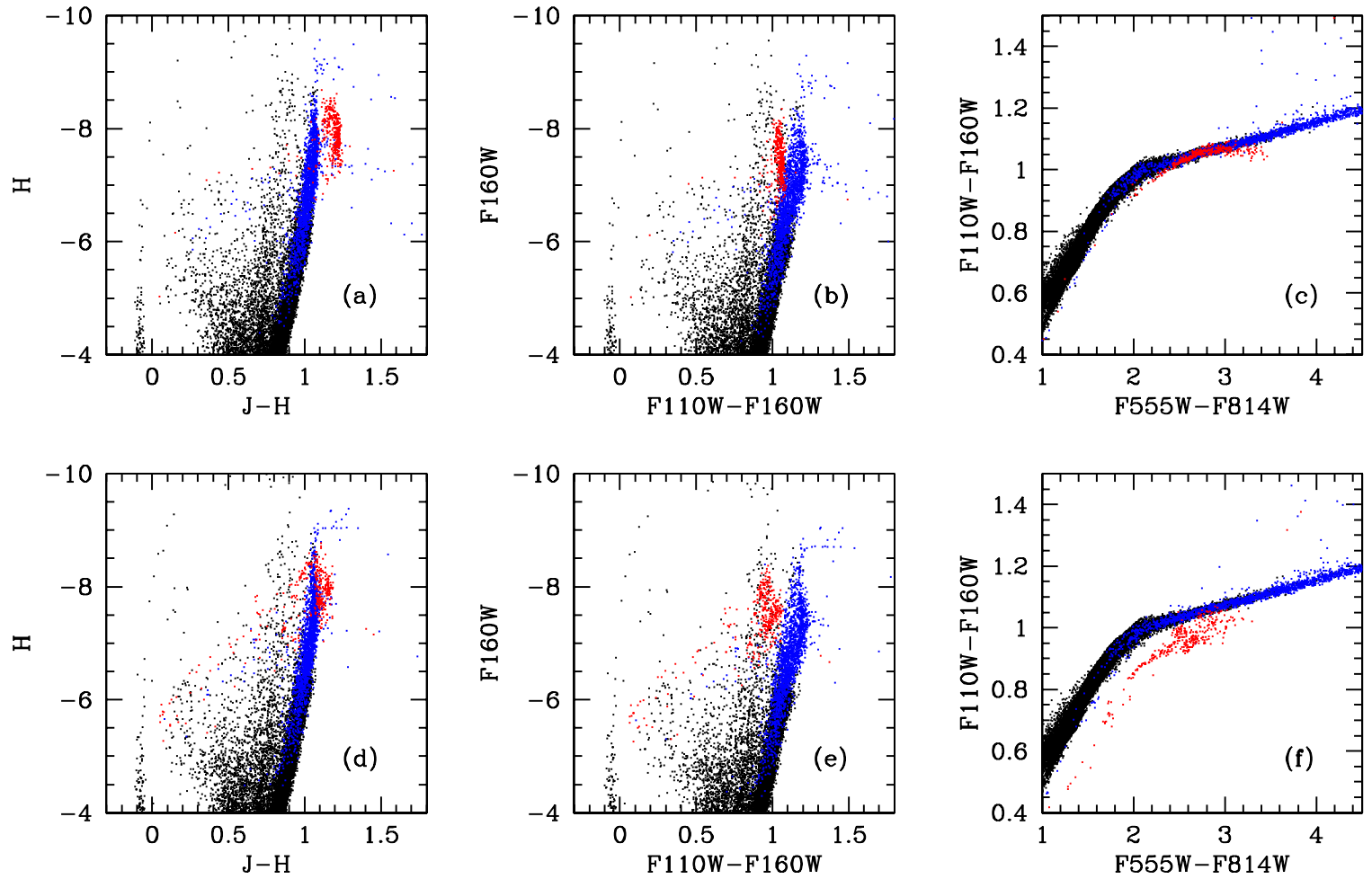

Figure 12. Simulated photometry for a model galaxy with a constant SFR and increasing metallicity (reaching $Z_{\odot}$ at the present day), made with the TRILEGAL code (Girardi et al. 2005; Girardi \& Marigo 2007). The TP-AGB population is color-coded by blue/red dots for O-rich/C-rich stars, respectively. The colors and magnitudes of C stars are derived from the model atmospheres by Loidl et al. (2001; top row, panels (a)-(c)) or by Aringer et al. (2009; bottom row, panels (d)-(f)). The colors of cool O-rich giants come from Fluks et al. (1994) model atmospheres. The left panels (a and d) show the expected $H$ vs. $J-H$ diagram for $2 \mathrm{MASS}$ filters, where $\mathrm{C}$ stars are found (as expected) to be slightly redder than O-rich stars. In the middle panels (b and e), however, $\mathrm{C}$ stars become bluer than the bulk of O-rich stars for the WFC3/IR $F 160 \mathrm{~W}$ vs. $F 110 \mathrm{~W}-F 160 \mathrm{~W}$ filter set, especially for the Aringer et al. (2009) models. The right panels (c and f) show the optical-IR color-color plots, comparable to Figure 15. For both models the simulations include extended tails of blue and faint TP-AGB stars, that correspond to the $\sim 15 \%$ of TP-AGB stars caught in their low-luminosity dips along thermally pulsing cycles, as well as the tails of extremely red stars at the late stages of high mass loss.

(A color version of this figure is available in the online journal.)
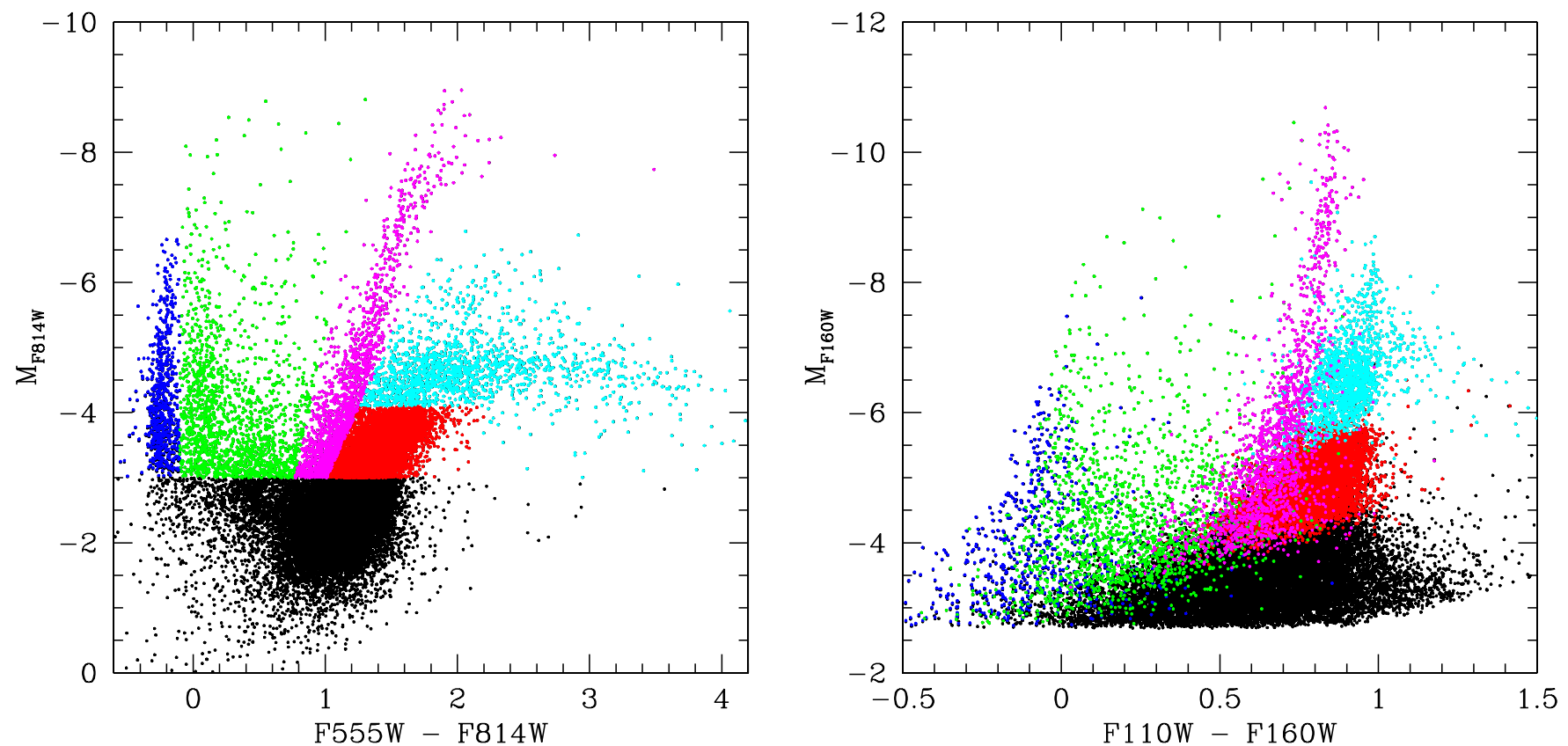

Figure 13. Comparison of CMD features in the optical (left) and the NIR (right) for IC2574-SGS. Points are color-coded according to their likely evolutionary phase (blue = main sequence; green = blue core helium burning; magenta = red core helium burning; red = red giant branch; cyan = asymptotic giant branch), as deduced from the optical CMD, for stars with $M_{F 814 W}<-2.5$ that are positionally well matched to stars in the NIR; unmatched or fainter stars are plotted in black.

(A color version of this figure is available in the online journal.) 
most active star formation show far more features than just the RGB and AGB (see targets IC2574-SGS (in I2574), NGC 0300WIDE1 (in N300), UGC4305-1 (in HoII), and UGC5139 (in HoI)). We now identify and briefly discuss these various features.

Star-forming NIR CMDs host a vertical sequence at $F 110 W-F 160 W \sim 0$ on the blue side of the CMD, made up of both main-sequence and BHeB stars. They also show a broad cloud of stars with colors intermediate between the main sequence and the RGB. These stars are most likely to be $\mathrm{BHeB}$ stars and other evolving massive stars. Note that only the most massive blue stars are detectable in the NIR. Such stars are rare, making it less likely that a "sequence" will be sufficiently well populated to appear as a distinct feature (see Figure 10), which reduces the clarity of the main sequence. In fields with on-going but low-intensity star formation (e.g., targets NGC 3741, NGC 7793-HALO-6, NGC 3077-PHOENIX), the "main sequence" manifests more as a blue edge to the cloud of points, rather than the narrow sequence seen in the optical.

To elucidate the correspondence between features in the optical and NIR CMDs, in Figure 13 we show CMDs for stars that have been matched between the NIR and optical photometry catalogs (Section 2.4) for IC2574-SGS, after correcting for foreground extinction and distance. The stars have been colorcoded by their likely evolutionary phase, as deduced from the optical. For this exercise, we use optical catalogs involving the $F 555 \mathrm{~W}$ filter, which provides good separation of CMD features, particularly between main-sequence and BHeB stars, and between RHeB and RGB stars. Stars that did not have highquality positional matches (coincident within 0.'07), or that were faint in $F 814 \mathrm{~W}$, are plotted in black. We note that the matching is unlikely to be perfect, and that some modest fraction of stars in the NIR catalog may have been incorrectly matched with stars in the optical catalog. This mismatching would be most likely in the crowded star-forming regions in this particular field, where slight errors in the alignment could still leave stars within the error radius for matching.

Figure 13 reveals a number of features. First, as expected, the main-sequence stars (in blue) crowd along the blue edge of the NIR CMD. However, the blue core helium-burning stars (in green) also contribute the blue vertical sequence, as well as filling the region between the NIR vertical blue sequence and the RGB. Thus, the lack of a clear BHeB sequence in the NIR is due primarily to its merging with the main sequence. On the red side of the NIR CMD, we see the RGB (red) and AGB (cyan) features discussed in Section 3.2.1, along with a modest horizontal tail of likely carbon-rich AGB stars at $M_{F 160 W} \sim-7$. In addition to the AGB stars seen in the CMDs of older stellar populations there is a brighter plume of AGB stars extending brightward of $M_{F 160 W} \sim-7.5$ and $M_{F 814 W} \sim-5$. This plume is likely due to the presence of more massive stars on the AGB, as a result of the strong star formation at $t<2 \mathrm{Gyr}$ in the IC2574-SGS field. Note also that the AGB stars fall on a welldefined sequence in the NIR CMD, but spread over a wide swath of color in the optical, as discussed above in Section 3.2.1.

The most remarkable feature in Figure 13 is the strong sequence of luminous RHeB stars. The color of the sequence is only slightly bluer than the AGB and RGB (by $\sim 0.2 \mathrm{mag}$ ), and extends to far brighter magnitudes. Indeed, the RHeB evolutionary phase is responsible for all of the most NIR luminous stars in IC2574. As can be seen in Figure 3, these luminous RHeB stars are tightly clustered within an active starforming region that features several young clusters spanning a

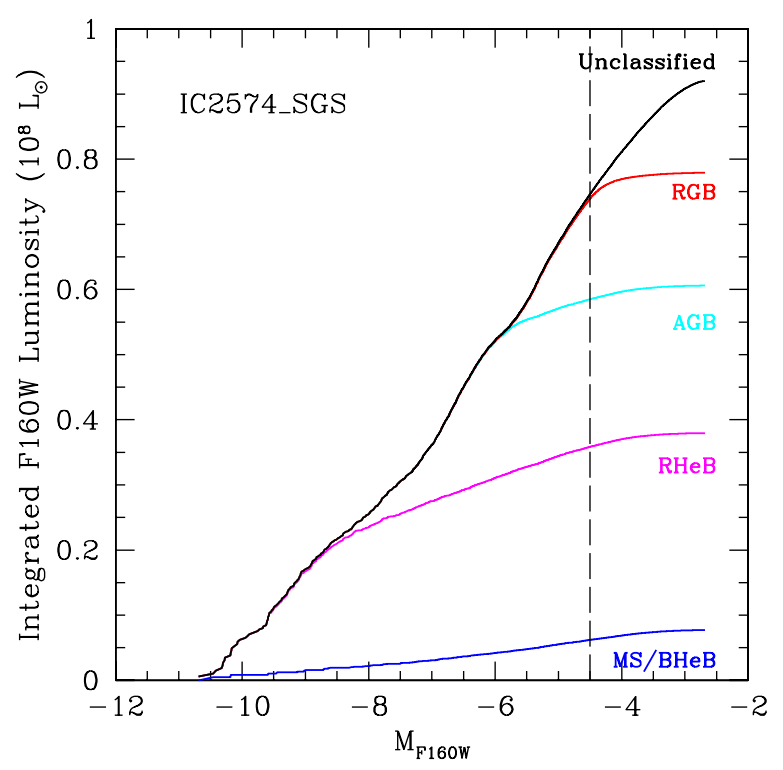

Figure 14. Integrated $F 160 \mathrm{~W}$ luminosity for IC2574-SGS, integrating from the bright to the faint end, for stars classified as in Figure 13. Each line contains the integrated total luminosity for stars of a given evolutionary phase or younger, such that the "AGB" line includes the luminosity in RHeB and MS/BHeB stars as well. "Unclassified" stars are those that were not reliably matched to stars in the optical catalog. Note that the contribution from RHeB stars dominates the NIR luminosity for the detected stars. The vertical line indicates the approximate completeness limit of the data; RGB stars are likely to be the dominant contributor to the luminosity below this limit, which will reduce the fraction of the luminosity due to RHeB or AGB stars. Note that the "RGB" classification likely includes some contamination from AGB and RHeB stars as well, since such stars cannot be reliably separated within the red giant branch.

(A color version of this figure is available in the online journal.)

100 Myr range of ages. Looking at the optical image (left), the youngest clusters in the region $(\lesssim 10 \mathrm{Myr})$ are still embedded in luminous H II regions, while the slightly older clusters are somewhat fainter, more diffuse, and lack extended line emission, although they are still quite blue, due to large concentrations of luminous BHeB stars. However, in the NIR (right), the relative luminosities of these clusters are reversed, such that the youngest clusters have negligible NIR emission, but the slightly older clusters have large concentrations of extremely luminous RHeB stars.

Thus, although much recent work has focused on the importance of the AGB phase to setting the NIR colors and luminosities of young stellar populations (Maraston et al. 2006; Henriques et al. 2011), it appears that RHeB stars can potentially be equally important. We demonstrate this in Figure 14, where we show the integrated luminosity of stars in different evolutionary phases. At the limit where our data become incomplete, RHeB stars are the dominant contribution, followed by AGB stars. The RGB, which is canonically assumed to dominate the NIR light, is only the third most important contributor to the luminosity at the completeness limit of our data. Note, however, that the fractional contribution of RGB stars will increase when the full range of stellar luminosities is considered; corrections for the missing stars are included in a companion paper by Melbourne et al. (2011), where the fraction of integrated light contributed by RHeB and AGB stars is quantified, showing that the RHeB can contribute up to $25 \%$ of the total light in $F 160 \mathrm{~W}$, and that current models underpredict the luminosity contribution of RHeB stars by up to a factor of four. 
The contribution of RHeB stars to the total luminosity will be strongest when the SFR has been elevated between 25 and $\sim 100 \mathrm{Myr}$ ago, which produces $\mathrm{RHeB}$ stars brighter than $M_{F 814 W} \sim-5$. This timescale is the same as the one over which UV emission is expected to be significant, and thus surveys that select for galaxies with high UV flux may also be selecting for galaxies with a significant RHeB population. Failure to account for the luminous, somewhat blue RHeB population would lead one to overestimate the mass-to-light ratio in the NIR, and to infer lower metallicities from the broadband colors. Unfortunately, the models of RHeB stars are even more uncertain than for AGB stars, and are known to produce erroneous colors and relative numbers of $\mathrm{BHeB}$ and RHeB at some metallicities (Langer \& Maeder 1995; DohmPalmer \& Skillman 2002; Gallart et al. 2005; McQuinn et al. 2011).

Our conclusions about the importance of RHeB stars are not significantly affected by uncertainties from foreground contamination. Unlike studies in the Magellanic Clouds, our fields cover small areas on the sky, which minimizes contributions from bright Milky Way stars. The lack of luminous contaminants can be seen empirically in Figure 2 (presented below), where we show the CMD of different subregions in the chip. We see essentially no luminous stars in the outer regions of small galaxies, where we are presumedly dominated by foreground stars and background galaxies (see HS117, for example). Predictions from the default TRILEGAL Milky Way model (Girardi et al. 2005) also suggest that we expect fewer than one contaminating star in each of the NIR CMDs plotted in Figure 9.

We also note that individual RHeB stars could easily be confused as individual stellar clusters, in images of galaxies that are nearby, but that are not sufficiently close to resolve individual stars. Due to their high luminosity and red colors, it may be difficult to distinguish single IR luminous RHeB stars from older stellar clusters with larger numbers of fainter RHeB stars.

\subsection{Color-Color Diagrams}

We can further explore the separation of different stellar evolutionary phases using the optical-NIR color-color diagram. We adopt the target IC1574-SGS as a test case, and use the matched catalogs from Figure 13 to generate an optical-NIR color-color diagram, color-coded as in Figure 13. The resulting color-color diagram is shown in Figure 15.

Figure 15 shows that the optical and NIR colors are highly correlated for colors bluer than $F 555 W-F 814 W \lesssim 1$.7. In this regime, the optical and NIR colors track each other extremely well, and are thus of little utility for separating phases of stellar evolution in data of this quality. At redder colors, however, there is noticeably decreased correlation between the optical and NIR colors, driven almost entirely by the behavior of the AGB population (cyan points in Figure 15). Although the bluest AGB stars follow a narrow sequence of optical-NIR colors, the optically redder AGB stars are far less well behaved. To first order, the NIR color saturates at $F 110 W-F 160 W \sim 1$ for a wide range of optical colors, with the exception of a likely population of extreme AGB stars (Blum et al. 2006). Qualitatively, it appears that the narrower blue AGB sequence bends over to fixed NIR color with increasing optical color. Beyond $F 555 W-F 814 W \gtrsim 1.7$, the narrow sequence appears to become embedded in a larger swath of red AGB stars, for which there is little correlation between optical and NIR color, and wide dispersion. There is no qualitative evidence

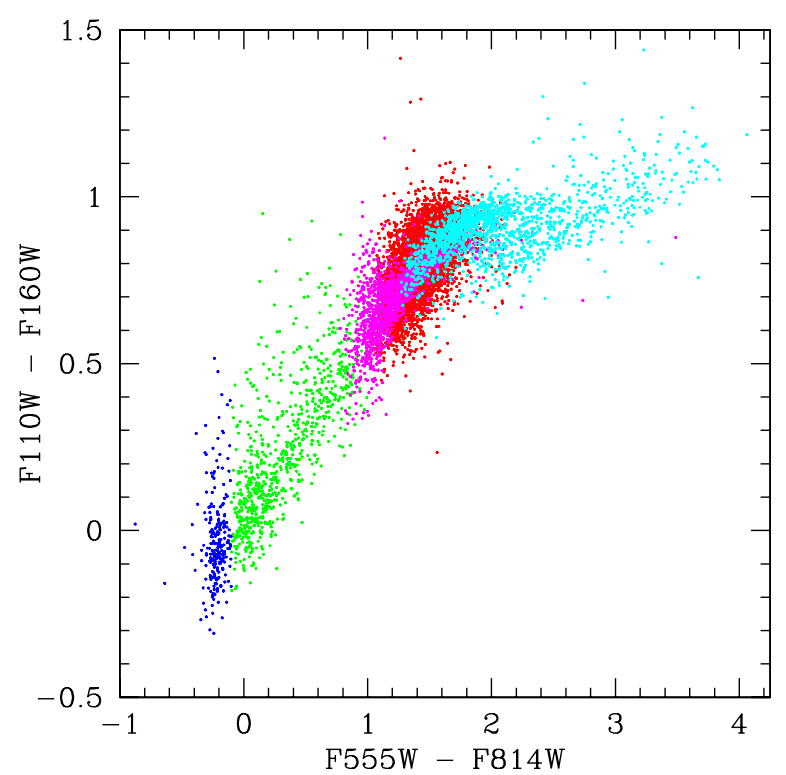

Figure 15. Optical vs. infrared color for stars within IC2574-SGS, color-coded as in Figure 13 (MS = dark blue; $\mathrm{BHeB}=$ green; $\mathrm{RHeB}=$ magenta; $\mathrm{RGB}=$ red; $\mathrm{AGB}=$ cyan). The AGB stars span a much wider range of optical colors, due to increased variability and reddening from circumstellar dust. The NIR colors of the AGB fall in a tight sequence for optical colors bluer than $F 555 W-F 814 W \lesssim 1.8$, but optically redder AGB stars show increased dispersion in their NIR colors. Only stars with magnitude uncertainties of less than $0.1 \mathrm{mag}$ are plotted.

(A color version of this figure is available in the online journal.)

for the narrower sequence continuing redward of $F 555 \mathrm{~W}-$ $F 814 W \gtrsim 2.2$.

We can further explore these different regimes of AGB behavior by qualitatively separating the AGB sequence into two regions and examining the behavior of the stars in the NIR CMD. Figure 16 shows the adopted division of the AGB population in color-color space (upper left), and the resulting NIR CMD (upper right). Although the division into two classes is somewhat arbitrary, and not motivated by a particular choice of stellar model, the adopted separation does appear to break the AGB population into two luminosity classes. As shown in the histograms in the lower left panels, the redder, highdispersion subpopulation is a constant fraction of the AGB stars brighter than $M_{F 160 W}<-6.8$; it also lacks lower luminosity AGB stars, and makes up fewer than $10 \%$ of the stars fainter than $M_{F 160 W}>-6.2$. In light of our selection criteria, this luminosity difference suggests that the higher luminosity, more massive AGB stars have systematically redder optical colors (lower right panel) and/or bluer NIR colors (with the exception of the extreme AGB stars redward of $F 110 W-F 160 W \gtrsim 1$ ). Brighter than $M_{F 160 W}<-6.8$, however, we see no noticeable difference in the NIR luminosities or median NIR colors of the two subpopulations (beyond the larger dispersion in the NIR color expected by the adopted division of the two populations in color-color space).

Although Figure 16 suggests that there are systematic luminosity-dependent differences in the optical-NIR colors of AGB stars, we currently lack a physical model that would allow us to make better motivated choices for dividing the AGB population on the basis of optical-NIR colors. It is tempting to think that the division we have made is helping to isolate carbon stars, based on the behavior seen in Figure 12. On the other hand, that same figure shows the large current uncertainties in the modeling at the relevant wavelengths. A more definitive ap- 

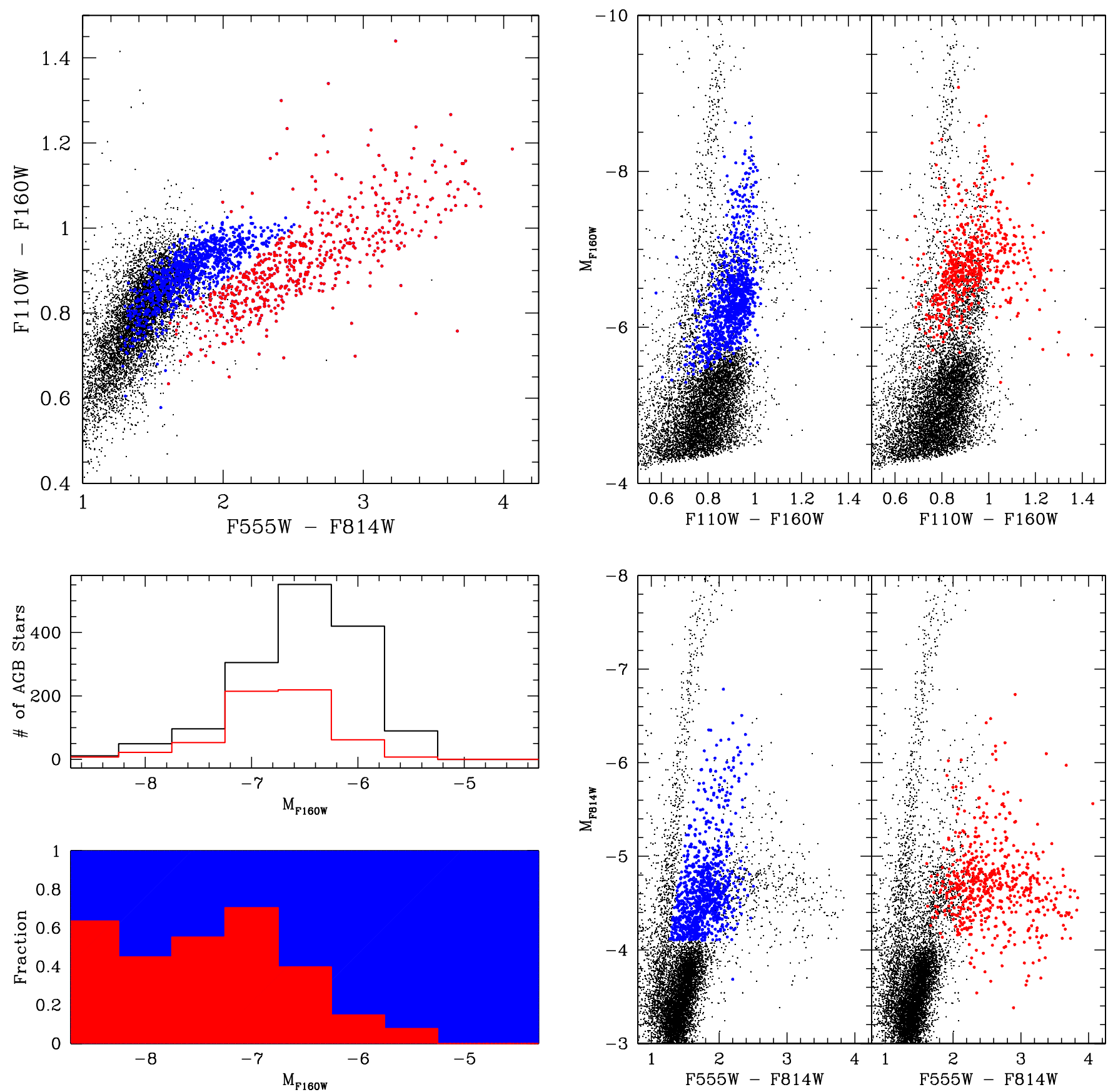

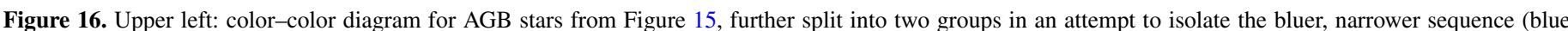

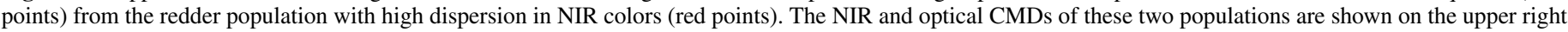

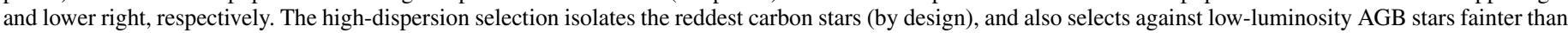

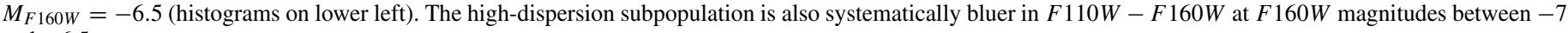
and -6.5 .

(A color version of this figure is available in the online journal.)

proach to isolating and modeling carbon stars will likely require observations at somewhat longer wavelengths than are currently possible with WFC3's IR channel, or the addition of narrowband imaging.

\subsection{Spatial Variations}

In addition to the field-to-field variations of the NIR CMDs discussed above, we also find strong spatial variations within individual fields. Such variation is naturally expected if young and intermediate-age populations contribute significantly to the NIR CMD. In the right-hand panels of Figure 2 we show the NIR CMDs in 16 subfields within each WFC3/IR frame, next to a color image of the same frame.

These grids show a number of features. First, one can see the effects of crowding. In the most well-populated fields, the depth of the CMD is strongly dependent on position, such that the densest subregions have the shallowest depth. Second, 
one can see significant spatial variations in the underlying stellar populations, particularly in fields that have significant amounts of recent star formation. Features due to the main sequence, RHeB, and AGB vary in strength and luminosity within individual fields. As an example, in NGC 0300-WIDE1 the stellar populations from the left side of the frame host a prominent main-sequence and luminous RHeB stars, whereas these populations are nearly absent from the lower right of the frame. Finally, CMD features due to young stellar populations typically appear with more clarity in the subregions than in the field as a whole (for example, note the sharp localization of the $\mathrm{RHeB}$ and BHeB in the upper left quadrants of IC2754-SGS, or in the relative population of the AGB and RGB across the field).

\section{TIP OF THE RED GIANT BRANCH IN THE NIR}

In the optical, the magnitude of the TRGB has become a widely used distance indicator for galaxies with resolved stellar populations (Lee et al. 1993; Sakai et al. 1996; Méndez et al. 2002; Karachentsev et al. 2006; Makarov et al. 2006). Its utility as a distance indicator rests on the relative insensitivity of the TRGB magnitude to age (for age $\gtrsim 3 \mathrm{Gyr}$ ) or metallicity (for $[\mathrm{Fe} / \mathrm{H}] \lesssim-0.5)$ in the $I$ band, where the bolometric magnitude of RGB stars typically peak (Lee et al. 1993).

In the NIR, however, the behavior of the TRGB magnitude is quite different. Theoretical models indicate that the magnitude of the TRGB should vary significantly with the properties of the underlying stellar population (Figure 2 of Salaris \& Girardi 2005), complicating the use of the NIR TRGB as a distance indicator. The variation in the NIR TRGB magnitude is known to be metallicity dependent for sub-solar metallicities $\left(\lesssim 0.5 Z_{\odot}\right)$, as has been demonstrated conclusively in data for globular clusters in the 2MASS filter set (Valenti et al. 2004). One can therefore potentially correct for the $\lesssim 1$ mag variation in the NIR TRGB absolute magnitude if the metallicity is known, allowing the TRGB to be used as a distance indicator in the NIR.

Unfortunately, this basic approach may not be effective in extragalactic systems, where the mean metallicity is uncertain, and the underlying stellar population is more complex. As shown in Figure 10 of Melbourne et al. (2010b), the presence of intermediate-age stars (1-2.5 Gyr) shifts the NIR TRGB to significantly fainter magnitudes. However, one could potentially diagnose the presence of this population through other means (for example, through a larger population of bright AGB stars), and correct for it. The metallicity dependence could likewise be corrected for as well; since metallicity directly affects the color of the RGB, it should be possible to empirically calibrate a relationship between the magnitude and color of the TRGB.

In this section, we derive the magnitude and color of the TRGB for our sample. We assume that the distances in Table 1 (based on $F 814 W$ TRGB measurements) are correct, and then derive the absolute magnitude of the NIR TRGB. In what follows, all magnitudes and colors have been corrected for the foreground extinction given in Table 1, assuming that $A_{F 110 W} / A_{\mathrm{V}}=0.33669$ and $A_{F 160 W} / A_{\mathrm{V}}=0.20443$ (Girardi et al. 2008, updated to included the latest in-flight calibrations for the WFC3 filter sets).

As a first step, in Figure 17 we plot the observed luminosity functions of the red extinction-corrected stars (dark black histogram), and their median color in magnitude bins (red line, plotted only for bins with more than 12 stars). Red stars are selected using a magnitude-dependent color cut to suppress the contribution of bluer young $\mathrm{BHeB}$ and main-sequence stars; brighter than the TRGB, we keep all stars redward of $F 110 W-F 160 W=0.5$, and fainter than the TRGB, we keep all stars redward of the diagonal line connecting $m=m_{\mathrm{TRGB}}, F 110 \mathrm{~W}-F 160 \mathrm{~W}=0.5$ with $m=m_{\mathrm{TRGB}}+4$, $F 110 W-F 160 W=-0.2$. This cut has no appreciable effect on any feature visible in the luminosity function. The luminosity functions have not been corrected for incompleteness, and we therefore expect them to roll over at faint magnitudes due solely to observational effects; we carry out a full correction for these effects in Melbourne et al. (2011), where we analyze the NIR luminosity contributed by different evolutionary phases.

Figure 17 shows that there is a clear variation in the absolute magnitude of the TRGB, relative to a fiducial TRGB absolute magnitude of $M_{F 160 W}=-5.7$, plotted as a vertical dotted line. Visual inspection shows that redder RGBs typically terminate at brighter absolute magnitudes; for these presumably metal-rich RGB stars, the bolometric flux peaks at redder wavelengths, increasing the flux in the NIR. In some cases one can also see the slight dip in the number of stars just brightward of the TRGB that was discussed in Section 3.2.1 (for example, see UGC4459, NGC 4163, NGC 3741, NGC 2976-DEEP, and IC2574-SGS for particularly obvious cases).

To quantify the variation in the TRGB, we measure the TRGB magnitude in $F 160 \mathrm{~W}$ using the edge-detection filter described in Méndez et al. (2002) applied to a Gaussiansmoothed luminosity function as in Sakai et al. (1996) and Seth et al. (2005). Although more sophisticated techniques exist (e.g., Makarov et al. 2006; Frayn \& Gilmore 2003), the TRGB of our sample is typically well populated and falls well above the photometric limit of the data, making our use of the widely used and calibrated edge-detection technique adequate for an initial measurement. We use the identical procedure as was used to measure the TRGB for the optical data (Dalcanton et al. 2009), making the $F 814 W$ and NIR TRGB directly comparable. Specifically, after extinction correcting all magnitudes and colors, we restrict our analysis to stars on the RGB by iteratively fitting a line to stars that are less than 1 mag fainter than the estimated TRGB and that have colors consistent with potential RGB stars $(0.6<F 110 W-F 160 W<1.1)$. We retain all stars that are within $2 \sigma$ of the fit to the RGB sequence toward the red, and within $1.5 \sigma$ to the blue; we use the more restrictive cut toward the blue to suppress the contribution from RHeB stars. The candidate RGB stars were then used to construct a Gaussian-smoothed luminosity function, which was then passed through an edge-detection filter.

The final TRGB magnitude and uncertainty was measured by executing 750 Monte Carlo bootstrap resampling trials. In each trial, additional Gaussian random errors were added to the stars' photometry, based on the magnitude of each star's photometric error. The TRGB for each trial was taken to be the magnitude corresponding to the peak of the edge-detection response filter within a $1 \mathrm{mag}$ interval around the likely TRGB. We then fit the histogram of the returned TRGB magnitudes with a Gaussian at $m_{\mathrm{TRGB}}$, taking the mean and width of the Gaussian to be the magnitude of the TRGB and its uncertainty. These quantities are converted to absolute magnitudes using the distance moduli in Table 1, adopted from the TRGB analysis in F814W.

Once the magnitude of the TRGB has been measured, we characterize the color of the TRGB using stars within 0.05 mag fainter than the TRGB; in the few cases where there are fewer than 15 stars within the adopted magnitude range, we increase 

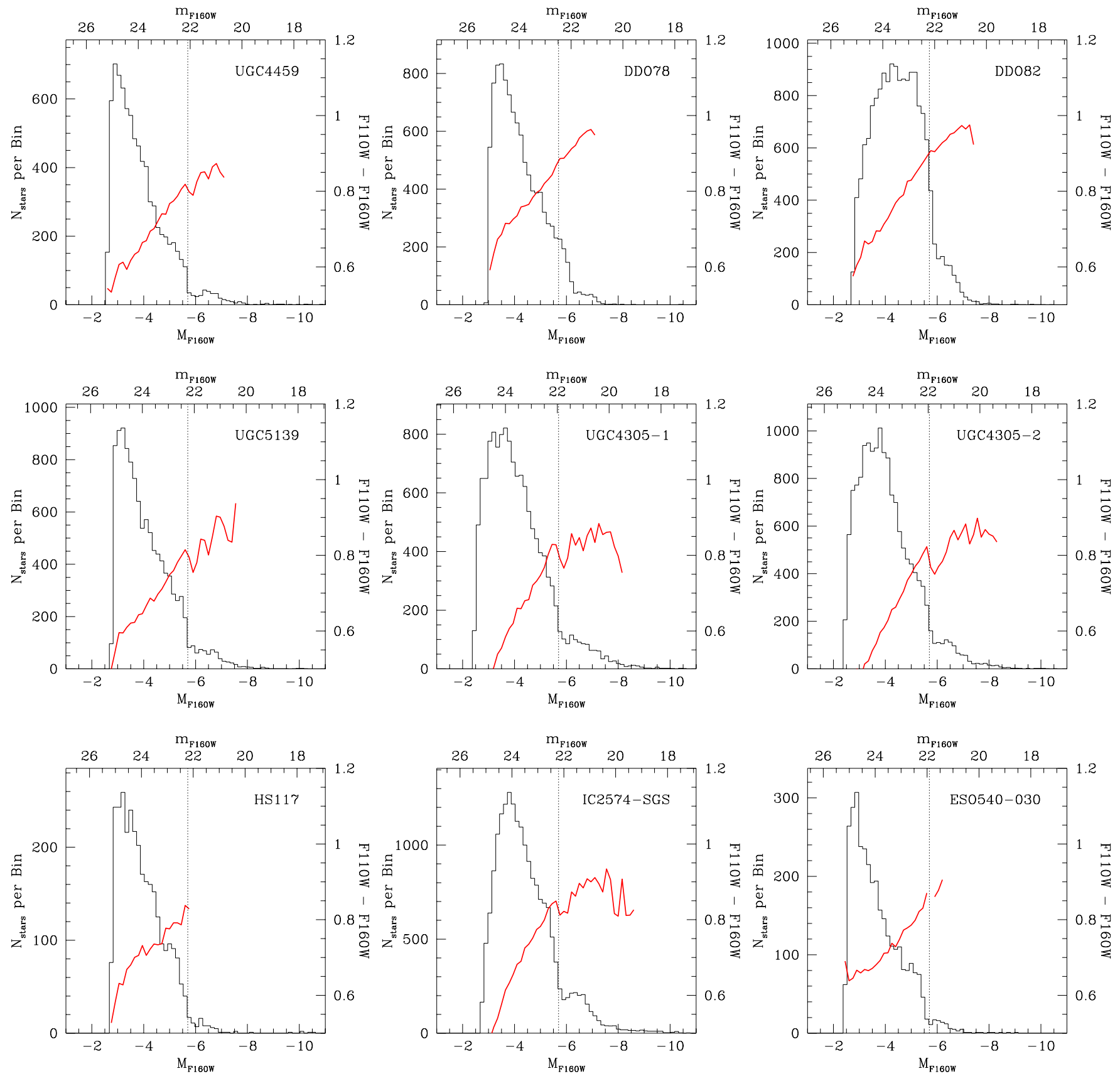

Figure 17. Luminosity functions in $F 160 \mathrm{~W}$ (black histogram, left axis) and median $F 110 \mathrm{~W}-F 160 \mathrm{~W}$ color (thick red line, right axis, for bins with $>12$ stars) for red stars ((a) DDO53; (b) DDO78; (c) DDO82; (d) HoI; (e) HoII; (f) HoII; (g) HS117; (h) I2574; (i) KDG2; (j) KDG63; (k) KDG73; (l) KKH37; (m) M81; (n) N300; (o) N404; (p) N2403; (q) N2403; (r) N2403; (s) N2976; (t) N3077; (u) N3741; (v) N4163; (w) N7793; (x) Sc22; (y) U8508; (z) UA292). All magnitudes have been extinction corrected, and distance moduli are as assumed in Table 1. The vertical dotted line indicates a fiducial TRGB magnitude of $F 160 \mathrm{~W}=-5.7$.

(A color version of this figure is available in the online journal.)

the range to $0.1 \mathrm{mag}$. We calculate the mean and standard deviation of these stars' colors using a biweight, iteratively clipping stars within $2 \sigma$ of the biweight mean, which visual inspection suggests captures all the width of the TRGB with acceptable rejection of $\mathrm{RHeB}$ stars.

Figure 18 shows the resulting absolute TRGB magnitude in $F 160 \mathrm{~W}$, as a function of the color of the TRGB. The data show the expected trend of brighter TRGB magnitudes for redder colors. This correlation can be well approximated as

$$
M_{\mathrm{TRGB}}(F 160 W)=-2.576(F 110 W-F 160 W)-3.496
$$

with the absolute magnitudes showing a scatter of $0.050 \mathrm{mag}$ around the mean.

Also shown in Figure 18 are expectations for two sets of theoretical models. The red line shows the magnitude and color of the TRGB for $10 \mathrm{Gyr}$ old Padova isochrones from the WFC3 extension of Girardi et al. (2008), spanning a range of metallicities. The blue line shows a fit to the magnitude and color of the TRGB for a series of isochrones supplied by Aaron Dotter, spanning a range of ages (6-14 Gyr, shown as asterisk with increasing point sizes for older TRGBs) and metallicities (from $[\mathrm{Fe} / \mathrm{H}]=-2.49$ (magenta) to $[\mathrm{Fe} / \mathrm{H}]=-0.99$ (green)). 

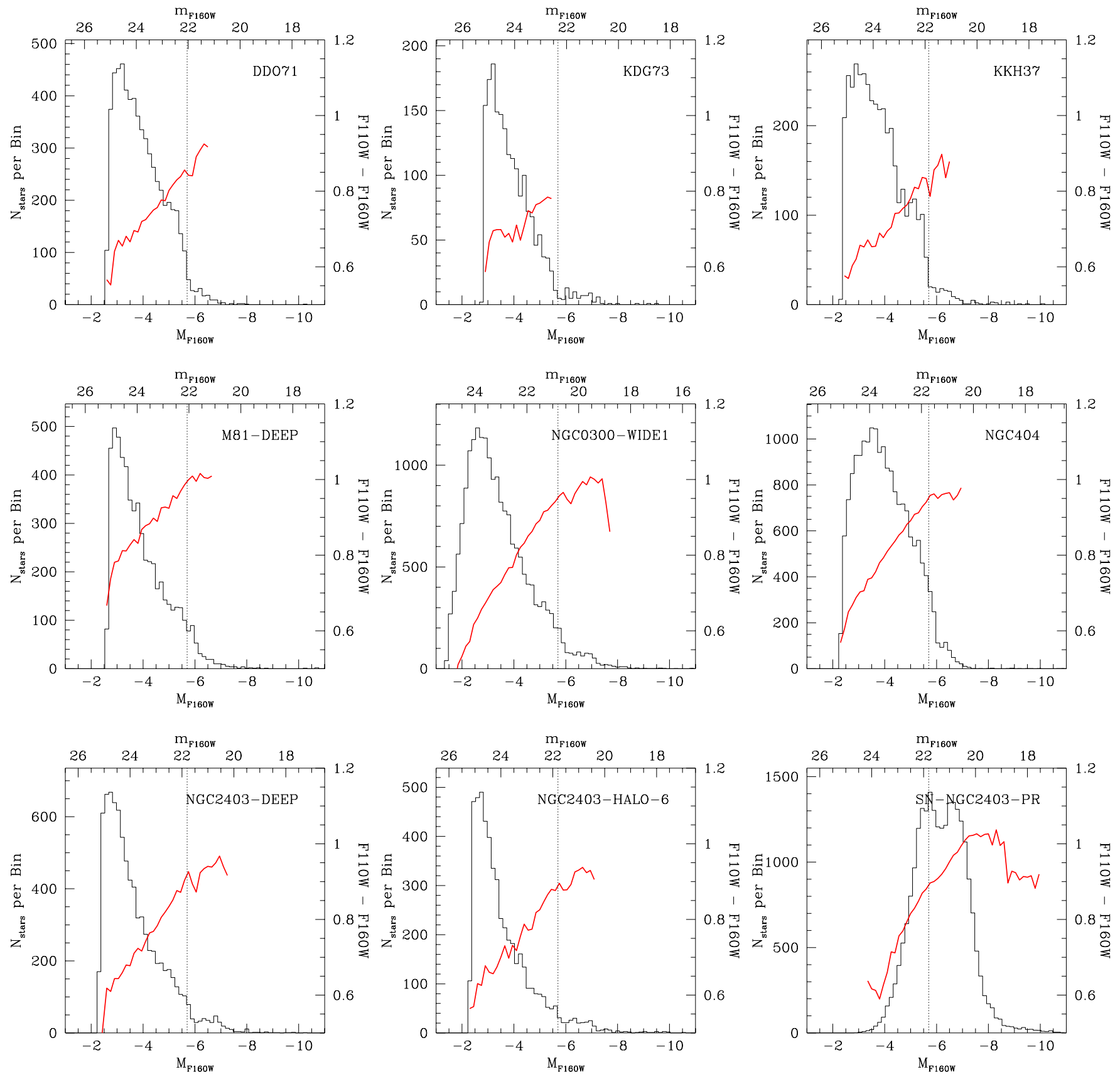

Figure 17. (Continued)

In general, the models show the same trend between TRGB color and magnitude seen in our data, suggesting that the bulk of the TRGB luminosity variation is driven by variations in metallicity. Similar behavior has been hinted at longer wavelengths for Spitzer Infrared Array Camera (IRAC) observations of galaxies within the Local Group (Boyer et al. 2009).

Although the slope of the fit to the Dotter isochrones is nearly identical to a weighted (in $X$ and $Y$ ) least-squares fit to the data (Equation (1)), the measured TRGBs are shifted to brighter magnitudes by a median of $0.099 \pm 0.027 \mathrm{mag}$ (or equivalently, to bluer colors by $0.043 \pm 0.012$ ), compared to the models. These offsets between the data and models are statistically significant, as judged by whether or not the mean residual in magnitude or color is significantly different from zero.
We have considered a number of possible origins for the offset between data and models in the left-hand plot of Figure 18. First, as a broad check on the consistency of our measurements, on the right side of Figure 18 we plot the correlation between our measured NIR TRGB color $(F 110 W-F 160 W)$ and the optical-NIR color of the TRGB inferred from the difference between our measurements of $m_{\text {TRGB }}$ in $F 814 W$ and $F 160 W$. These two colors appear to be highly correlated, as expected (Figure 15). However, they also show a small offset from the Dotter isochrones, such that the median observed optical-NIR color of the TRGB is 0.04 mag redder than the Dotter isochrones, with a semi-interquartile range of \pm 0.037 . If the source of the offset in the $M_{\text {TRGB }}(F 160 W)$ versus $F 110 W-F 160 W$ relation were primarily a bias toward bluer measurements of the mean 

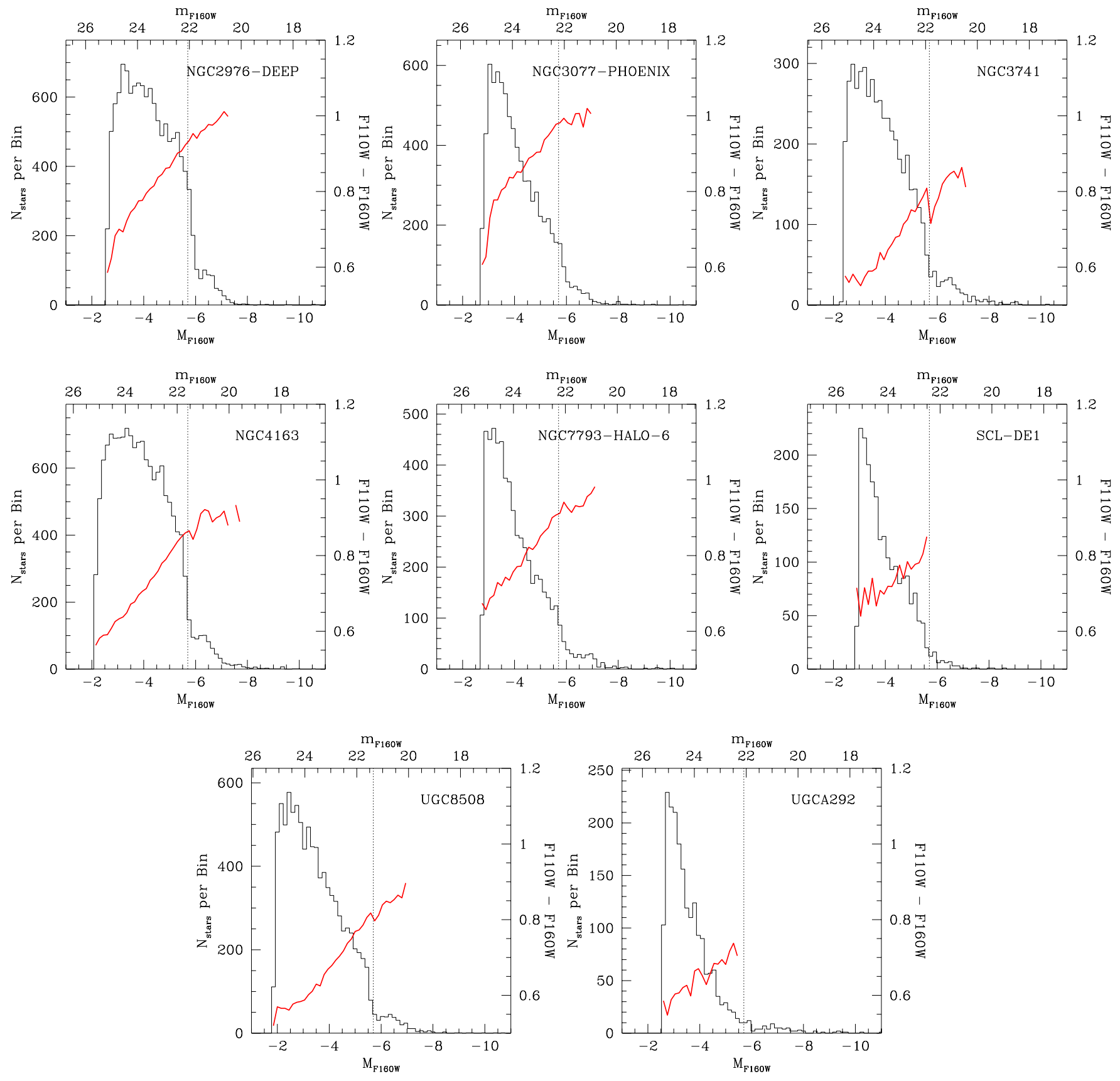

Figure 17. (Continued)

color of the TRGB, then the data should be offset to bluer colors in this diagram as well, rather than to redder $F 110 \mathrm{~W}-F 160 \mathrm{~W}$ colors as is observed. Thus, the offset between data and models does not appear to be due a mismeasurement of the TRGB color.

Alternatively, if the source of the offset were primarily a bias toward brighter measurements of the $F 160 \mathrm{~W}$ TRGB magnitude, then the data would be shifted to redder values of $m(F 814 W)-m(F 160 W)$ in this diagram. We do indeed see a redward shift between the data and the models for $m(F 814 W)-m(F 160 W)$. However, the amplitude of this shift is a factor of 2.5 times smaller than needed to explain the offset observed in $M_{\text {TRGB }}(F 160 W)$ versus $F 110 W-F 160 W$.
The final explanation we consider is if the offset results from a mismatch between the filter calibrations and the filter throughputs adopted by the models (such that measured F160W magnitudes are brighter than would be inferred for the models). If so, then both the $m(F 814 W)-m(F 160 W)$ and $F 110 W-$ $F 160 W$ values would be shifted to redder colors compared to the models. However, this shift would move points along the mean relation, rather than perpendicular to it, and thus is unlikely to produce significant offsets. This possibility is consistent with the direction and the magnitudes of the offsets in both diagrams shown in Figure 18.

As a second approach to assessing the origin of the offset, we have included measurements of the TRGB from globular 

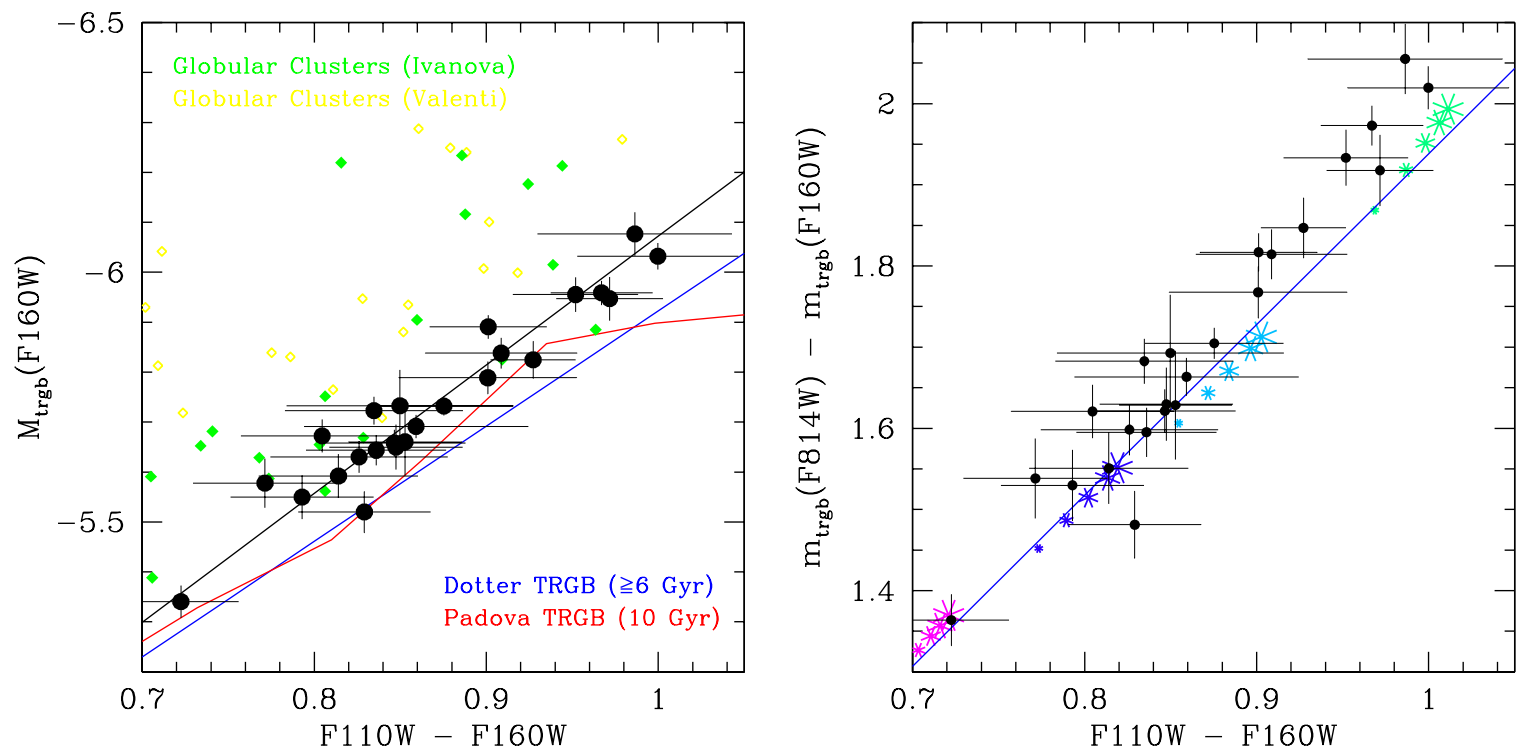

Figure 18. Left: absolute $F 160 \mathrm{~W}$ magnitude of the TRGB as a function of $F 110 \mathrm{~W}-F 160 \mathrm{~W}$ color, for data in this sample (solid black circles). Also shown are loci from the isochrone models of Girardi et al. (2008) for a $10 \mathrm{Gyr}$ RGB with a range of metallicities (solid red line; updated to the WFC3/IR filter set), and a fit to isochrone models provided by A. Dotter (2010, private communication; blue solid line). Diamonds are data from globular clusters (solid green points are from Ivanov \& Borissova 2002; open yellow points are from Valenti et al. 2004, 2007). Our measurements are slightly offset from the models by roughly 0.15 mag brighter in $F 160 \mathrm{~W}$, or 0.1 mag bluer in color. Right: magnitude difference between the optical $F 814 \mathrm{~W}$ (from Dalcanton et al. 2009) and NIR $F 160 \mathrm{~W}$ TRGB, as a function of the measured $F 110 \mathrm{~W}-F 160 \mathrm{~W}$ color of the TRGB. Lines and symbols are the same as in the left-hand plot. Individual points for the Dotter isochrone TRGB values are plotted for ages of $6,8,10,12$, and $14 \mathrm{Gyr}$ (increasing asterisk sizes) and metallicities of $[\mathrm{Fe} / \mathrm{H}]=-2.49,[\alpha / \mathrm{Fe}]=0.40, Y=0.245(\mathrm{magenta}),[\mathrm{Fe} / \mathrm{H}]=-1.99$, $[\alpha / \mathrm{Fe}]=0.40, Y=0.246$ (blue), $[\mathrm{Fe} / \mathrm{H}]=-1.49,[\alpha / \mathrm{Fe}]=0.20, Y=0.246$ (cyan), and $[\mathrm{Fe} / \mathrm{H}]=-0.99,[\alpha / \mathrm{Fe}]=0.20, Y=0.249$ (green).

(A color version of this figure is available in the online journal.)

clusters in Figure 18. These measurements are from Ivanov \& Borissova (2002; solid green diamonds) and the Valenti et al. (2004, 2007) samples (open yellow diamonds, ${ }^{24}$ restricting the sample to those with $A_{H}<0.5$ ). Both data sets were originally taken in $J$ and $H$. We transformed these data to $F 160 \mathrm{~W}$ and $F 110 W$ using the following relations:

$$
\begin{aligned}
F 160 W-H= & 0.2031+0.401(J-H-0.9) \\
& +0.3498(J-H-0.9)^{2}
\end{aligned}
$$

and

$$
\begin{aligned}
J-H= & 0.9418+0.841(F 110 W-F 160 W-1.0) \\
& -0.9053(F 110 W-F 160 W-1.0)^{2},
\end{aligned}
$$

which were derived by fitting the magnitudes and colors of the TRGB for colors redder than $F 110 W-F 160 W<1.15$ in the Padova models; these transformations are good to $<0.001 \mathrm{mag}$ across this range.

The globular cluster data tend to be shifted to brighter magnitudes and/or bluer colors than our measurements, and are even more offset from the models (in both the WFC3/IR and native $J+H$ filter sets). The offsets are more pronounced for the Valenti sample, which appears to be biased somewhat brightward compared to other data, including two clusters in common with the Ivanov \& Borissova (2002) sample, ${ }^{25}$ and three bulge clusters in common with the sample of Chun et al.

\footnotetext{
24 Including updates from http://www.bo.astro.it/ GC/ir_archive/.

25 The two clusters are NGC 6441, which is 0.07 mag brighter in $F 160 \mathrm{~W}$ and 0.08 mag bluer in the Valenti et al. (2004) sample, and NGC 6624, which is 0.12 mag brighter and has an identical color in the Valenti et al. (2004) sample. Both of these clusters have relatively high degrees of foreground extinction, which increases the likelihood of differences between independent analyses.
}

(2010). We note, however, that measurements of the TRGB can be difficult in globular clusters, which frequently have uncertain distances, sparsely populated RGBs at bright magnitudes, and ambiguous distinctions between bright RGB and faint AGB stars. Furthermore, the extinction corrections are typically quite large for the comparison globular cluster sample; even with our restriction on the foreground extinction, the median extinction is larger than $0.3 \mathrm{mag}$ in $H$ for the Valenti sample.

We have also considered that photometric biases may be responsible for the small offsets in our TRGB magnitudes and/or colors. For example, the Monte Carlo process used to evaluate our uncertainties artificially increases the photometric error (during randomization of magnitudes) and potentially biases $m_{\text {TRGB }}$ by scattering stars preferentially above the tip. In practice, however, the effect of the added noise is negligible, since the photometric uncertainties are extremely small at $m_{\text {TRGB }}$. The effect of these biases has been explored extensively in Madore \& Freedman (1995), and at our typical S/N level of $\mathrm{S} / \mathrm{N} \sim 50$, we expect negligible magnitude biases due to photometric errors. We likewise have considered the effect of crowding on our measurements, and find that it too is unlikely to explain the offset. As shown in Figure 8, our photometric measurements are essentially unbiased in the median, with less than a 0.005 mag shift at faint magnitudes. Crowding does produce a slight tail to brighter magnitudes, due to undetected stars contaminating the flux of the resolved stars. However, at the typical magnitude of the TRGB, fewer than $3 \%$ of fake stars have magnitudes that would be brighter than expected from photometric uncertainty alone. In addition, we see no correlation between the degree of offset from the models and the number of stars in the field.

We also have explored population differences as being a source of the offset between the data and the models. Variations 
in the mean stellar age can affect the magnitude of the TRGB, while contamination from RHeB and AGB stars can change both the color and magnitude of the TRGB. However, we found no significant impact from these effects, based on the lack of correlation between the amplitude of the offset and the fraction of stars formed in different age ranges.

We are left without any satisfying explanation for this small offset between the data and the models. At this point, our best guess is that it results from some combination of small uncertainties in the current WFC3/IR calibrations (although these improved significantly over the course of this program), in the filter throughputs adopted by the models, or the models themselves. Indeed, Cassisi (2010, Figure 2) shows significant systematic variations among models for the predicted bolometric magnitudes of the TRGB. For now, any attempt to use $F 160 \mathrm{~W}$ measurements for the TRGB should acknowledge that there may be an underlying $10 \%$ systematic uncertainty in the calibration.

\section{LUMINOSITY FUNCTIONS}

In the above analysis, we identify the TRGB as the point where there is a sharp peak in the edge-detection algorithm that also corresponds with the largest drop in the number of stars. However, the edge-detection algorithm frequently identifies other sharp transitions in the luminosity function that correspond to smaller changes in the absolute number of stars with magnitude.

To explore these effects, in Figure 19 we plot all galaxy luminosity functions, relative to the observed magnitude of the TRGB. All magnitudes are relative to the TRGB magnitude given in Table 3 . The light lines are color-coded according to each galaxy's rank when sorted by the fraction of star formation the galaxy has experienced in the most recent Gyr. The heavy lines show the average luminosity function when the galaxies are sorted by rank into four bins of recent star formation, with equal numbers of galaxies per bin; red, magenta, blue, and black lines go from lowest to highest fraction of recent star formation $\left(\left\langle f_{0-1 \mathrm{Gyr}}\right\rangle=0.001,0.04,0.07,0.09\right.$, respectively). All luminosity functions have been normalized to have the same total number of stars in the bins between 0.25 and 0.75 mag fainter than the TRGB. We do not analyze the luminosity function at fainter magnitudes, where the varying depths, photometric uncertainties, and distances among the galaxy population make the averaging procedure invalid. These luminosity functions include only stars that lie within the extrapolated slope and width of the RGB, and may miss the reddest, most luminous $\mathrm{RHeB}$ stars.

The average luminosity functions in Figure 19 show clear systematic deviations that correlate with the fraction of star formation in the most recent gigayear. These deviations are most obvious brighter than the TRGB, where elevated recent star formation produces a larger population of $\mathrm{RHeB}$ and luminous AGB stars, extending to brighter magnitudes. As noted previously in Section 3.2.2 and as quantified in Melbourne et al. (2011), this population of luminous stars can lead to significant reductions in the NIR mass-to-light ratio, on much shorter timescales than discussed for AGB stars alone.

There are also subtle features close to the TRGB itself, some of which correlate with the recent SFR. In particular, the amplitude of the discontinuity at the TRGB is less pronounced when recent star formation is more prominent, most likely due to contamination from younger stellar populations all along

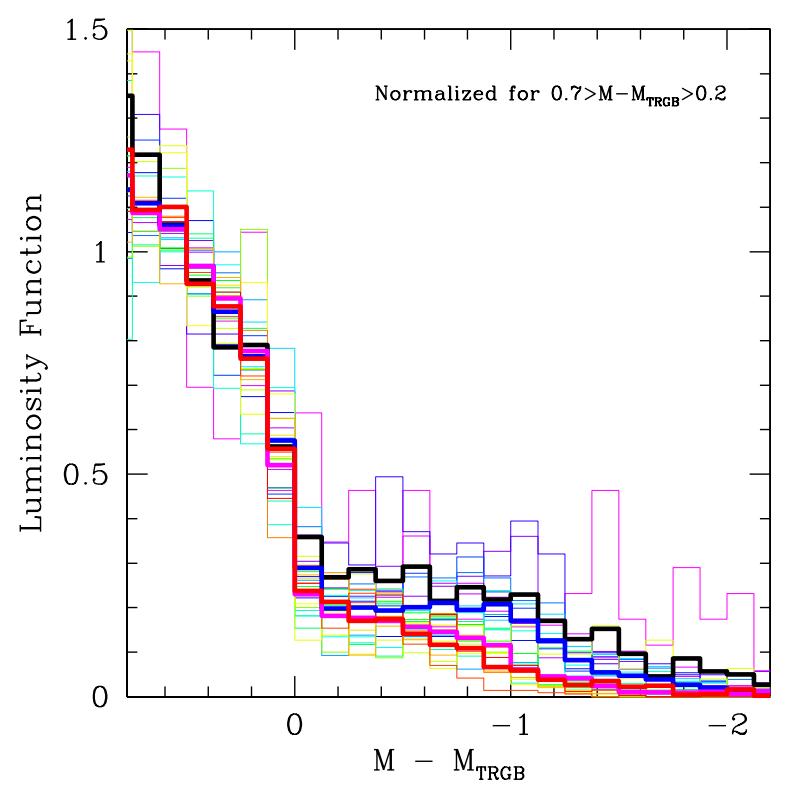

Figure 19. RGB luminosity functions for all galaxies, color-coded by their rank when sorted by the fraction of star formation in the most recent $1 \mathrm{Gyr}$ (light lines, with redder colors indicating older mean stellar ages). Dark lines show the average luminosity function of galaxies, sorted by rank into four bins of recent star formation, with equal numbers of galaxies per bin; red, magenta, blue, and black lines go from lowest to highest fraction of recent star formation $\left(\left\langle f_{0-1 \mathrm{Gyr}}\right\rangle=0.012,0.038,0.071,0.089\right.$, respectively). All luminosity functions are for stars found within $1.5 \sigma$ of the line fit to the upper 1 mag of the RGB, and are normalized to have the same number of stars within the bins between 0.25 and 0.75 mag fainter than the TRGB. All magnitudes are relative to the TRGB magnitude given in Table 3.

(A color version of this figure is available in the online journal.)

the RGB sequence and its extension to brighter magnitudes. This contamination will tend to reduce the reliability of the NIR TRGB distance measurements for actively star-forming galaxies.

\section{METALLICITY AND THE STRUCTURE OF THE RGB}

For two decades it has been known that the slope of the NIR RGB correlates strongly with metallicity for globular clusters (e.g., Davidge et al. 1992; Cohen \& Sleeper 1995; Kuchinski et al. 1995; Ferraro et al. 2000). While this correlation allows the slope of the NIR RGB to be used as a reddening-free diagnostic of metallicity for globular clusters (for an example, see Ferraro et al. 2006), it is not clear if the relationships used for uniaged globular clusters can be routinely applied to the complex stellar populations found in galaxies, where age variations can also affect the structure of the RGB, and where observations typically produce lower quality photometry due to unavoidable crowding errors.

We now evaluate whether the NIR RGB slope can be used effectively as a metallicity indicator for complex stellar populations. We first characterize the RGB using the measured median color as a function of magnitude (red lines from Figure 17; see Section 4). We presume that the observed variation in color is dominated by variations in metallicity, based on the greater sensitivity of RGB color to metallicity compared to age (upper right of Figure 10). Thus, while we cannot directly compare the RGB slope to metallicity, we can use the color of the TRGB as a proxy. 
Table 3

TRGB Measurements

\begin{tabular}{|c|c|c|c|c|c|c|c|c|}
\hline $\begin{array}{l}\text { Catalog } \\
\text { Name }\end{array}$ & $\begin{array}{l}\text { Target } \\
\text { Name }\end{array}$ & $\begin{array}{c}m-M \\
(F 814 W)\end{array}$ & $A_{\mathrm{V}}$ & $\begin{array}{c}N_{\text {stars }} \\
\text { (TRGB fit) }\end{array}$ & $\begin{array}{l}\text { Mean } \\
\text { Color }\end{array}$ & $\begin{array}{c}m_{\text {TRGB }} \\
\text { (raw) }\end{array}$ & $\begin{array}{c}m_{\text {TRGB }} \\
(F 160 W)\end{array}$ & $\begin{array}{c}M_{\mathrm{TRGB}} \\
(F 160 W)\end{array}$ \\
\hline KDG63 & DDO71 & 27.74 & 0.303 & 1024 & 0.853 & 22.142 & $22.080 \pm 0.064$ & $-5.660 \pm 0.064$ \\
\hline DDO78 & DDO78 & 27.82 & 0.066 & 1438 & 0.901 & 22.045 & $22.031 \pm 0.027$ & $-5.789 \pm 0.027$ \\
\hline DDO82 & DDO82 & 27.90 & 0.133 & 5268 & 0.909 & 22.089 & $22.062 \pm 0.028$ & $-5.838 \pm 0.028$ \\
\hline KDG2 & ESO540-030 & 27.61 & 0.072 & 481 & 0.829 & 22.105 & $22.090 \pm 0.040$ & $-5.520 \pm 0.040$ \\
\hline HS117 & HS117 & 27.91 & 0.359 & 480 & 0.836 & 22.340 & $22.266 \pm 0.025$ & $-5.644 \pm 0.025$ \\
\hline $\mathrm{I} 2574$ & IC2574-SGS & 27.90 & 0.112 & 3967 & 0.859 & 22.232 & $22.209 \pm 0.019$ & $-5.691 \pm 0.019$ \\
\hline KDG73 & KDG73 & 28.03 & 0.056 & 273 & 0.771 & 22.464 & $22.452 \pm 0.045$ & $-5.578 \pm 0.045$ \\
\hline KKH37 & ККH37 & 27.56 & 0.231 & 659 & 0.848 & 21.957 & $21.910 \pm 0.026$ & $-5.650 \pm 0.026$ \\
\hline M81 & M81-DEEP & 27.77 & 0.249 & 594 & 0.986 & 21.744 & $21.693 \pm 0.043$ & $-6.077 \pm 0.043$ \\
\hline N300 & NGC 0300-WIDE1 & 26.50 & 0.039 & 1410 & 0.972 & 20.561 & $20.553 \pm 0.026$ & $-5.947 \pm 0.026$ \\
\hline $\mathrm{N} 2403$ & NGC 2403-DEEP & 27.50 & 0.124 & 751 & 0.927 & 21.701 & $21.675 \pm 0.036$ & $-5.825 \pm 0.036$ \\
\hline $\mathrm{N} 2403$ & NGC 2403-HALO-6 & 27.50 & 0.124 & 356 & 0.901 & 21.635 & $21.609 \pm 0.016$ & $-5.891 \pm 0.016$ \\
\hline N2976 & NGC 2976-DEEP & 27.76 & 0.224 & 2495 & 0.952 & 21.851 & $21.805 \pm 0.034$ & $-5.955 \pm 0.034$ \\
\hline N3077 & NGC 3077-PHOENIX & 27.92 & 0.208 & 945 & 1.000 & 21.931 & $21.888 \pm 0.025$ & $-6.032 \pm 0.025$ \\
\hline N3741 & NGC 3741 & 27.55 & 0.077 & 801 & 0.814 & 21.974 & $21.958 \pm 0.023$ & $-5.592 \pm 0.023$ \\
\hline N404 & NGC 404 & 27.42 & 0.181 & 2691 & 0.967 & 21.498 & $21.461 \pm 0.021$ & $-5.959 \pm 0.021$ \\
\hline N4163 & NGC 4163 & 27.29 & 0.062 & 2594 & 0.875 & 21.571 & $21.558 \pm 0.014$ & $-5.732 \pm 0.014$ \\
\hline N7793 & NGC 7793-HALO-6 & 27.96 & 0.060 & 648 & 0.949 & 21.930 & $21.917 \pm 0.020$ & $-6.043 \pm 0.020$ \\
\hline $\mathrm{Sc} 22$ & SCL-DE1 & 28.11 & 0.046 & 377 & 0.793 & 22.570 & $22.560 \pm 0.037$ & $-5.550 \pm 0.037$ \\
\hline HoII & UGC4305-1 & 27.65 & 0.098 & 1845 & 0.850 & 21.937 & $21.917 \pm 0.071$ & $-5.733 \pm 0.071$ \\
\hline HoII & UGC4305-2 & 27.65 & 0.098 & 2258 & 0.835 & 21.947 & $21.927 \pm 0.025$ & $-5.723 \pm 0.025$ \\
\hline DDO53 & UGC4459 & 27.79 & 0.118 & 932 & 0.805 & 22.142 & $22.118 \pm 0.031$ & $-5.672 \pm 0.031$ \\
\hline HoI & UGC5139 & 27.95 & 0.153 & 1742 & 0.826 & 22.351 & $22.320 \pm 0.029$ & $-5.630 \pm 0.029$ \\
\hline U8508 & UGC8508 & 27.06 & 0.047 & 1110 & 0.847 & 21.412 & $21.402 \pm 0.024$ & $-5.658 \pm 0.024$ \\
\hline UA292 & UGCA292 & 27.79 & 0.048 & 184 & 0.722 & 22.459 & $22.450 \pm 0.022$ & $-5.340 \pm 0.022$ \\
\hline
\end{tabular}

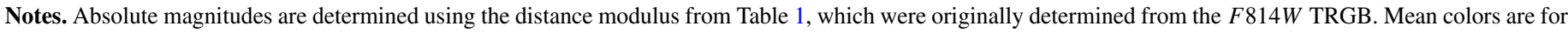

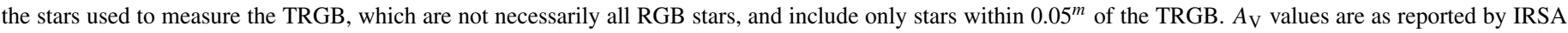

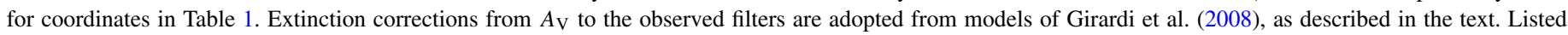

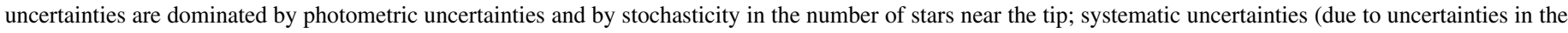

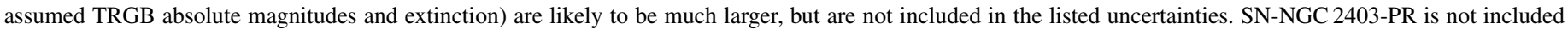
due to large crowding errors.

\subsection{TRGB Color and the Luminosity-Metallicity Relationship}

To demonstrate the connection between metallicity and NIR color of the TRGB, in Figure 20, we plot the color of the TRGB as a function of each galaxy's extinction-corrected $B_{T}$ magnitude (upper left) and NIR luminosity (upper right; $3.6 \mu \mathrm{m}$ luminosity taken from Dale et al. (2009), assuming that the Sun has a flux of $14.71 \mathrm{Jy}$ at a distance of $10 \mathrm{pc}$ ). The data show a clear relationship between galaxy luminosity and TRGB color, such that $F 110 W-F 160 W=0.605-0.0181 M_{\mathrm{B}_{\mathrm{T}}}$ and $F 110 \mathrm{~W}-F 160 \mathrm{~W}=0.431+0.0518 \log _{10} L_{3.6 \mu}$, with an rms of 0.046 and 0.039 , respectively. Because of the well-known mass-metallicity relationship (e.g., Lee et al. 2006, and references therein), one would expect higher metallicities in more massive, luminous galaxies, which should then manifest itself as more luminous galaxies having redder TRGBs. Figure 20 shows this expected behavior, suggesting that the TRGB color does indeed correlate with metallicity.

Further support for the connection between TRGB color and metallicity comes from the lower left panel, where we plot the NIR TRGB color as a function of the metallicity inferred from the oldest age bin of the SFH (derived from the optical photometry using MATCH; Melbourne et al. 2011). We see a tight correlation between NIR TRGB color and metallicity, following the relationship $F 110 W-F 160 W=$ $1.093+0.192[\mathrm{Fe} / \mathrm{H}]$ with an rms of 0.050 . Note, however, that one expects this correlation to be tight, given that the metallicity sensitivity of the CMD fits comes largely from the color of the TRGB.

\subsection{RGB Slope as a Metallicity Indicator}

Having verified the relationship between TRGB color and metallicity, we now investigate the correlation between RGB slope and the TRGB color. On the left side of Figure 21 we plot linear fits to the RGB, restricted to 2 mag fainter than the TRGB. ${ }^{26}$ The plot shows the expected variation of the magnitude of the TRGB with color, such that redder RGBs extend to brighter magnitudes (Figure 18).

On the right side of Figure 21 we plot the slope of the linear fit compared to the color of the fit at the magnitude of the TRGB. In general, there are no systematic statistically significant trends across the whole sample. There is a $\sim 25 \%$ dispersion in the mean slope for colors between $0.8 \lesssim F 110 W-F 160 W \lesssim 1$, with no apparent correlation. This suggests that the observed RGB slope is unlikely to be a useful metallicity indicator in the presence of the dispersion in age, metallicity, and photometric errors within individual extragalactic systems.

The only possible manifestations of the known correlation between NIR RGB slope and metallicity can be seen at the extremes. Of the four galaxies with the most vertical RGBs, three also have the bluest colors. Two of these three (UA292 and Sc22) do indeed to have very low metallicity populations,

\footnotetext{
26 The RGB slope in globular clusters is typically measured down to near the horizontal branch (e.g., Kuchinski et al. 1995). However, in extragalactic systems one typically cannot resolve more than 2-3 mag of the RGB, due to crowding.
} 

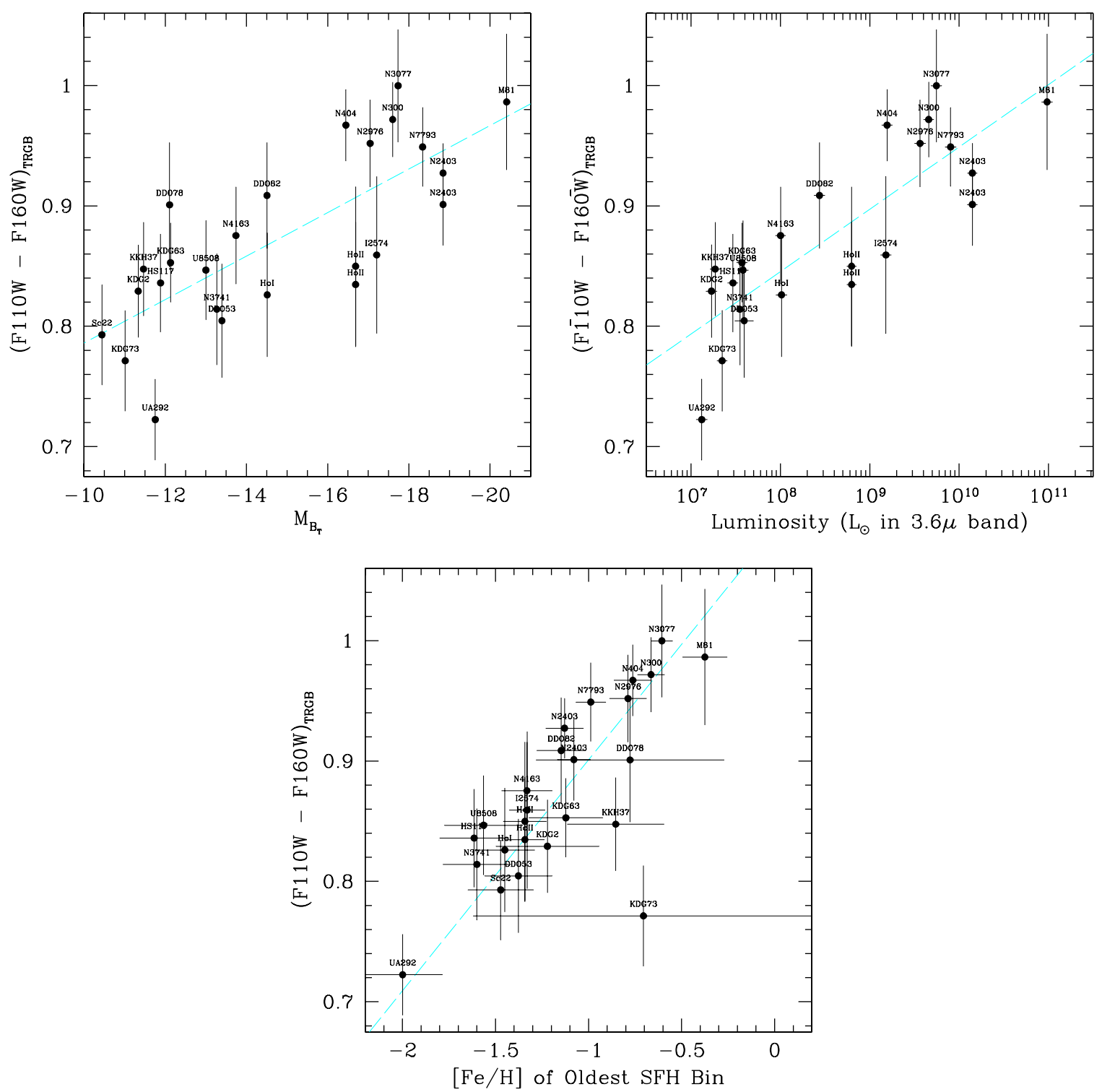

Figure 20. Upper left: $F 110 \mathrm{~W}-F 160 \mathrm{~W}$ color of the TRGB, plotted against the absolute magnitude of the host galaxy in the $B_{T}$ filter. Error bars indicate the characteristic width of the RGB near the tip (Table 3). There is a statistically significant correlation between color and galaxy luminosity (Spearman rank correlation coefficient of -0.75 , giving a less than $0.001 \%$ chance of occurring by chance), which is likely to be driven by the underlying mass-metallicity relationship for galaxies. Many of the galaxies that fall below the mean relationship have high recent star formation rates, which may bias the luminosity high (due to low mass-to-light ratios) or the RGB color blueward (due to contamination from RHeB and AGB stars, or younger mean RGB ages). Upper right: $F 110 W-F 160 W$ color of the TRGB, plotted against the solar luminosity of the host galaxy in the Spitzer $3.6 \mu \mathrm{m}$ bandpass (Dale et al. 2009). Again, a strong color-luminosity trend is apparent (Spearman rank correlation coefficient of 0.82). The plot excludes DDO78 and Sc22, which are undetected in the Spitzer $3.6 \mu \mathrm{m}$ bandpass. Lower left: $F 110 W-F 160 W$ color of the TRGB as a function the $[\mathrm{Fe} / \mathrm{H}]$ metallicity inferred from the oldest bin of the star formation history adopted by Melbourne et al. (2011). There is a strong trend (Spearman rank correlation coefficient of 0.54 , giving a less than $0.3 \%$ chance of occurring by chance, decreasing to $0.03 \%$ if KDG73 is excluded) driven by the fact that the color of the RGB is one of the strongest discriminant of metallicities in the CMD. Dashed lines indicate the fitting equations given in the text.

(A color version of this figure is available in the online journal.)

based upon the lack of curvature in the optical RGB. In contrast, the remaining galaxy (KDG73) does not have a particularly old globular-like stellar population, nor does the optical CMD suggest a particularly low metallicity (based upon the observed curvature in the optical RGB). Instead, this galaxy appears to have a substantial younger AGB population which may be pulling the RGB to bluer colors and altering its morphology (see Figure 10).

The other extreme outliers are HoII and IC2574, both of which have particularly shallow RGB slopes. However, both of these galaxies also have dramatic $\mathrm{RHeB}$ populations, which are likely shifting the base of the RGB fit to bluer colors, artificially flattening the RGB slope.

In summary, there appears to be little chance that the slope of the NIR RGB can be used to assess the metallicity of extragalactic systems with current data quality; the existing trends appear to be weak at best, and outliers due to contributions from AGB and RHeB stars are not uncommon. One may have better success by combining the NIR with the optical to produce an optical-NIR CMD with a wide color baseline (i.e., similar to the widely used $V-K$ color), or with using a more sophisticated method to characterize the RGB slope. 

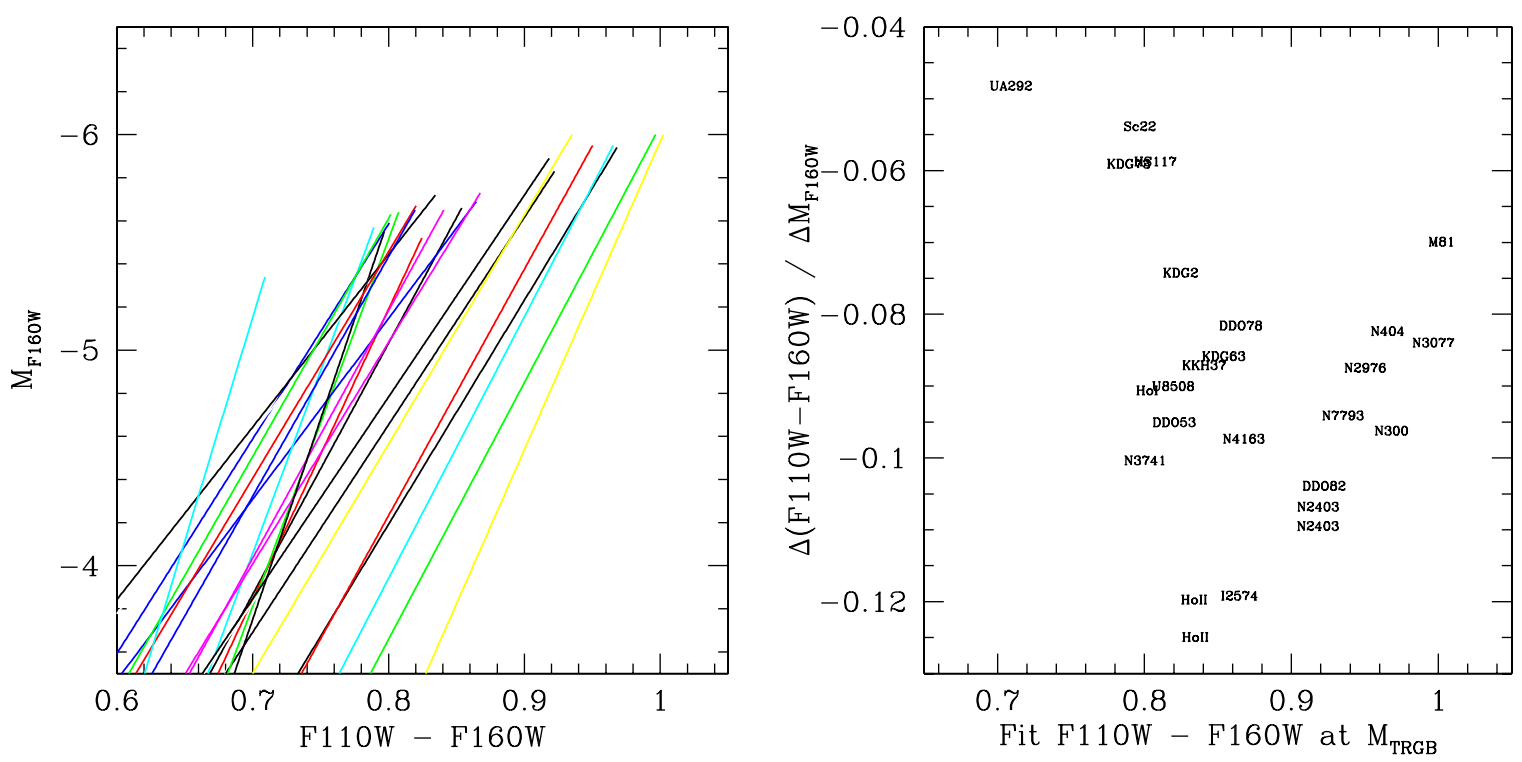

Figure 21. Left: fits to the median color of the top 2 mag of the RGB, using data from Figure 17. Colors have been randomly assigned to individual galaxies. All quantities have been corrected for extinction. Right: slopes of the fits shown in the left-hand panel, as a function of the color of the fits at the TRGB. There are no obvious systematic trends across the whole sample, suggesting that the presence of complex stellar populations can mask any intrinsic variation in the shape of the RGB with metallicity. There are, however, suggestive trends at the extremes. First, the three bluest RGBs make up three of the four steepest RGBs, suggesting a possible detection of variations in RGB shape at very low metallicity. Second, the three fields with extremely prominent RHeB sequences (IC2574 and two fields in HoII) have the three shallowest RGBs, suggesting that the presence of RHeB stars are biasing the slope of the RGB by pulling the base to bluer colors. These same galaxies are also outliers in the luminosity-metallicity plots in Figure 20.

(A color version of this figure is available in the online journal.)

\section{CONCLUSIONS}

This work represents the first step in characterizing the NIR properties of complex stellar populations for galaxies spanning a wide range of metallicity and SFH. We have presented CMDs in one of the most efficient and commonly used WFC3/IR filter pairs, allowing one to assess the contribution of individual stars to the integrated light in these bandpasses.

In all cases, the CMDs show a dominant population of RGB stars, as expected. They also show clear sequences of AGB stars, with only a modest contribution of extremely red AGB stars. These features are characteristic of old and intermediateage star formation. In galaxies with more recent star formation, we also find a dramatic sequence of RHeB stars, extending to much brighter magnitudes than the AGB sequence. These stars are associated with recent star formation (20-500 Myr), and in some cases are a major contributor to the NIR flux. They also have colors that are similar to the underlying AGB and RGB population, making their presence difficult to diagnose from broadband colors alone. These $\mathrm{RHeB}$ stars therefore present a significant uncertainty in adopting a NIR mass-to-light ratio when analyzing unresolved galaxies or interpreting their NIR colors. We quantify the contribution of both $\mathrm{RHeB}$ and AGB stars in Melbourne et al. (2011).

We have used the observed CMDs to empirically calibrate the NIR TRGB magnitude as a function of the metallicity-sensitive $F 110 W-F 160 W$ color, allowing these standard HST filters to be used as distance indicators in the WFC3/IR and JWST era. We find that there is a strong correlation between color and the absolute magnitude of the TRGB, as expected from models and previous observations of globular clusters. However, we find that the relationship is offset by $\sim 0.05-0.1 \mathrm{mag}$ from the prediction of current isochrone models. Analysis of the origin of this offset points to a residual uncertainty in either the WFC3/IR zero points or the theoretical models.

We also explore the structure of the RGB and AGB sequences, highlighting possible variations with the age and metallicity of the underlying stellar population. We see a clear age-dependent variation in the luminosity function of red stars, due to increasing numbers of luminous AGB stars with increasing intermediateage star formation. We are unable to detect any metallicity dependence in the slope of the RGB, however, which prevents the slope of the NIR RGB from being used as a reddening-free metallicity indicator.

We also present an analysis of scattered light in the WFC3/IR detector. We have found a rapidly varying scattered light component that affects images taken at low angles to a bright Earth limb. We discuss how this scattered light may affect photometry, particularly when fitting a series of non-destructive reads with a linear function.

In a series of subsequent papers, we will be using the data presented here to refine theoretical models of RHeB and AGB stars, to assess contributions of carbon stars, to empirically calibrate the fractional luminosity due to AGB and RHeB stars, and to improve SFHs at intermediate ages.

The authors are very happy to acknowledge helpful discussions with Ben Weiner about scattered light in WFC3/IR. Aaron Dotter is warmly thanked for supplying theoretical RGB sequences, as is Jason Kalirai for providing information helpful for WFC3/IR calibration and processing. As always, we thank Alison Vick for her support in scheduling and executing these observations. The anonymous referee is thanked for providing useful, detailed comments on this very long paper. J.J.D. thanks the Max Planck Institute für Astronomie for their hospitality while writing part of this paper. L.G. and P.M. 
acknowledge support from contract ASI-INAF I/009/10/0. This work was supported by the Space Telescope Science Institute through SNAP-11719, and used additional data products produced through GO-10915 and AR-10945.

\section{REFERENCES}

Aaronson, M., Cohen, J. G., Mould, J., \& Malkan, M. 1978, ApJ, 223, 824 Aringer, B., Girardi, L., Nowotny, W., Marigo, P., \& Lederer, M. T. 2009, A\&A, 503, 913

Battinelli, P., \& Demers, S. 2005, A\&A, 434, 657

Battinelli, P., \& Demers, S. 2009, A\&A, 493, 1075

Bertelli, G., Bressan, A., Chiosi, C., Fagotto, F., \& Nasi, E. 1994, A\&AS, 106, 275

Blum, R. D., Mould, J. R., Olsen, K. A., et al. 2006, AJ, 132, 2034

Boyer, M. L., Skillman, E. D., van Loon, J. T., Gehrz, R. D., \& Woodward, C. E. 2009, ApJ, 697, 1993

Boyer, M. L., Srinivasan, S., van Loon, J. Th., et al. 2011, AJ, 142, 103

Brinchmann, J., \& Ellis, R. S. 2000, ApJ, 536, L77

Bundy, K., Ellis, R. S., \& Conselice, C. J. 2005, ApJ, 625, 621

Cassisi, S. 2010, in IAU Symp. 262, Stellar Populations-Planning for the Next Decade, ed. G. Bruzual \& S. Charlot (Cambridge: Cambridge Univ. Press), 13

Castellani, V., Degl'Innocenti, S., Girardi, L., et al. 2000, A\&A, 354, 150

Chiosi, C., Bertelli, G., \& Bressan, A. 1992, ARA\&A, 30, 235

Chun, S.-H., Kim, J.-W., Shin, I.-G., et al. 2010, A\&A, 518, A15

Cirasuolo, M., McLure, R. J., Dunlop, J. S., et al. 2010, MNRAS, 401, 1166

Cohen, J. G., \& Sleeper, C. 1995, AJ, 109, 242

Conroy, C., \& Gunn, J. E. 2010, ApJ, 712, 833

Conselice, C. J., Bundy, K., Ellis, R. S., et al. 2005, ApJ, 628, 160

Dahlen, T., Mobasher, B., Somerville, R. S., et al. 2005, ApJ, 631, 126

Dalcanton, J. J., Williams, B. F., Seth, A. C., et al. 2009, ApJS, 183, 67

Dale, D. A., Cohen, S. A., Johnson, L. C., et al. 2009, ApJ, 703, 517

Davidge, T. J. 2010, ApJ, 718, 1428

Davidge, T. J., Harris, W. E., Bridges, T. J., \& Hanes, D. A. 1992, ApJS, 81, 251

Dohm-Palmer, R. C., \& Skillman, E. D. 2002, AJ, 123, 1433

Dohm-Palmer, R. C., Skillman, E. D., Mateo, M., et al. 2002, AJ, 123, 813

Dolphin, A. E. 2000, PASP, 112, 1383

Dolphin, A. E. 2002, MNRAS, 332, 91

Ferraro, F. R., Montegriffo, P., Origlia, L., \& Fusi Pecci, F. 2000, AJ, 119, 1282

Ferraro, F. R., Valenti, E., \& Origlia, L. 2006, ApJ, 649, 243

Fluks, M. A., Plez, B., The, P. S., et al. 1994, A\&AS, 105, 311

Frayn, C. M., \& Gilmore, G. F. 2003, MNRAS, 339, 887

Frogel, J. A., Mould, J., \& Blanco, V. M. 1990, ApJ, 352, 96

Gallart, C., Zoccali, M., \& Aparicio, A. 2005, ARA\&A, 43, 387

Girardi, L., Bertelli, G., Bressan, A., et al. 2002, A\&A, 391, 195

Girardi, L., Dalcanton, J., Williams, B., et al. 2008, PASP, 120, 583

Girardi, L., Groenewegen, M. A. T., Hatziminaoglou, E., \& da Costa, L. 2005, A\&A, 436, 895

Girardi, L., \& Marigo, P. 2007, in ASP Conf. Ser. 378, Why Galaxies Care About AGB Stars: Their Importance as Actors and Probes, ed. F. Kerschbaum, C. Charbonnel, \& R. F. Wing (San Francisco, CA: ASP), 20

Girardi, L., \& Salaris, M. 2001, MNRAS, 323, 109
Girardi, L., Williams, B. F., Gilbert, K. M., et al. 2010, ApJ, 724, 1030

Grocholski, A. J., \& Sarajedini, A. 2002, AJ, 123, 1603

Groenewegen, M. A. T. 1999, in IAU Symp. 191, Asymptotic Giant Branch Stars, ed. T. Le Bertre, A. Lebre, \& C. Waelkens (Cambridge: Cambridge Univ. Press), 535

Groenewegen, M. A. T. 2007, in ASP Conf. Ser. 378, Why Galaxies Care About AGB Stars: Their Importance as Actors and Probes, ed. F. Kerschbaum, C. Charbonnel, \& R. F. Wing (San Francisco, CA: ASP), 433

Gullieuszik, M., Held, E. V., Rizzi, L., et al. 2007, A\&A, 467, 1025

Gullieuszik, M., Held, E. V., Rizzi, L., et al. 2008, MNRAS, 388, 1185

Henriques, B., Maraston, C., Monaco, P., et al. 2011, MNRAS, 415, 3571

Ivanov, V. D., \& Borissova, J. 2002, A\&A, 390, 937

Karachentsev, I. D. 2005, AJ, 129, 178

Karachentsev, I. D., Dolphin, A., Tully, R. B., et al. 2006, AJ, 131, 1361

Karachentsev, I. D., Grebel, E. K., Sharina, M. E., et al. 2003, A\&A, 404, 93

Karachentsev, I. D., Karachentseva, V. E., Huchtmeier, W. K., \& Makarov, D. I. 2004, AJ, 127, 2031

Karakas, A. I., Lattanzio, J. C., \& Pols, O. R. 2002, PASA, 19, 515

Kriek, M., Labbe, I., Conroy, C., et al. 2010, ApJ, 722, L64

Kuchinski, L. E., Frogel, J. A., Terndrup, D. M., \& Persson, S. E. 1995, AJ, 109,1131

Langer, N., \& Maeder, A. 1995, A\&A, 295, 685

Lee, H., Skillman, E. D., Cannon, J. M., et al. 2006, ApJ, 647, 970

Lee, M. G., Freedman, W. L., \& Madore, B. F. 1993, ApJ, 417, 553

Loidl, R., Lançon, A., \& Jørgensen, U. G. 2001, A\&A, 371, 1065

Madore, B. F., \& Freedman, W. L. 1995, AJ, 109, 1645

Makarov, D., Makarova, L., Rizzi, L., et al. 2006, AJ, 132, 2729

Maraston, C., Daddi, E., Renzini, A., et al. 2006, ApJ, 652, 85

Marigo, P., \& Aringer, B. 2009, A\&A, 508, 1539

Marigo, P., \& Girardi, L. 2007, A\&A, 469, 239

Marigo, P., Girardi, L., Bressan, A., et al. 2008, A\&A, 482, 883

McQuinn, K. B. W., Skillman, E. D., Dalcanton, J., et al. 2011, ApJ, 740, 48

Melbourne, J., Williams, B., Dalcanton, J., et al. 2010a, ApJ, 712, 469

Melbourne, J., Williams, B., Dalcanton, J., et al. 2010b, ApJ, 712, 469

Melbourne, J., Williams, B., Dalcanton, J., et al. 2012, ApJ, submitted

Méndez, B., Davis, M., Moustakas, J., et al. 2002, AJ, 124, 213

Nikolaev, S., \& Weinberg, M. D. 2000, ApJ, 542, 804

Olsen, K. A. G., Blum, R. D., Stephens, A. W., et al. 2006, AJ, 132, 271

Rejkuba, M., da Costa, G. S., Jerjen, H., Zoccali, M., \& Binggeli, B. 2006, A\&A, 448, 983

Sakai, S., Madore, B. F., \& Freedman, W. L. 1996, ApJ, 461, 713

Salaris, M. 2002, in ASP Conf. Ser. 274, Observed HR Diagrams and Stellar Evolution, ed. T. Lejeune \& J. Fernandes (San Francisco, CA: ASP), 50

Salaris, M., \& Girardi, L. 2005, MNRAS, 357, 669

Saracco, P., Fiano, A., Chincarini, G., et al. 2006, MNRAS, 367, 349

Schlegel, D. J., Finkbeiner, D. P., \& Davis, M. 1998, ApJ, 500, 525

Seth, A. C., Dalcanton, J. J., \& de Jong, R. S. 2005, AJ, 130, 1574

Stancliffe, R. J. 2006, MNRAS, 370, 1817

Thronson, H. A., Jr., \& Greenhouse, M. A. 1988, ApJ, 327, 671

Tully, R. B., Rizzi, L., Dolphin, A. E., et al. 2006, AJ, 132, 729

Valdes, F. G., Campusano, L. E., Velasquez, J. D., \& Stetson, P. B. 1995, PASP, 107, 1119

Valenti, E., Ferraro, F. R., \& Origlia, L. 2004, MNRAS, 354, 815

Valenti, E., Ferraro, F. R., \& Origlia, L. 2007, AJ, 133, 1287

van Dokkum, P. G., Franx, M., Kriek, M., et al. 2008, ApJ, 677, L5

Weisz, D. R., Dalcanton, J. J., Williams, B. F., et al. 2011, ApJ, 739, 5

Williams, B. F., Dalcanton, J. J., Johnson, L. C., et al. 2011, ApJ, 734, 22 